\title{
Mechanism of the Visible Light-Mediated Gold-Catalyzed Oxyarylation Reaction of Alkenes
}

\author{
Qi Zhang, ${ }^{a, b}$ Zhen-Qi Zhang, ${ }^{\text {a }}$ Yao Fu, ${ }^{a}$ Hai-Zhu Yu ${ }^{* b}$ \\ ${ }^{a}$ Department of Chemistry, University of Science and Technology of China, Hefei 230026 \\ ${ }^{b}$ Department of Chemistry and Centre for Atomic Engineering of Advanced Materials, \\ Anhui University, Hefei 230601 \\ Emails: yuhaizhu@ahu.edu.cn
}

Table of Contents

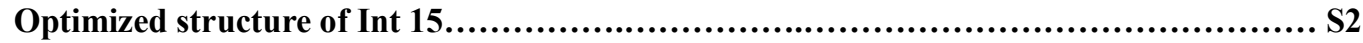

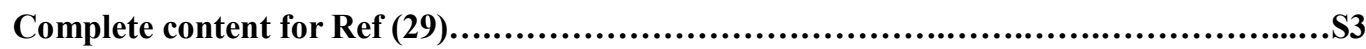

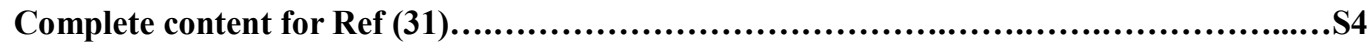

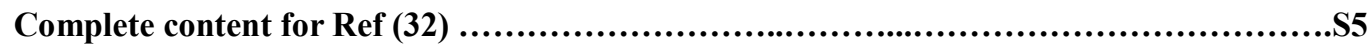

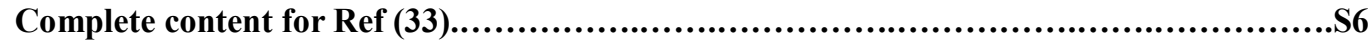

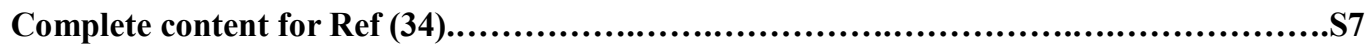

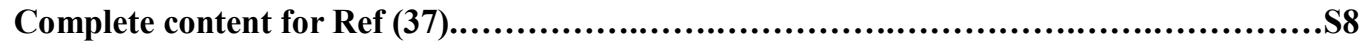

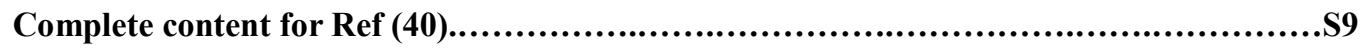

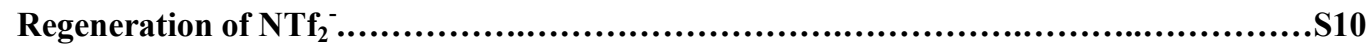

Complete content for Ref (41) …................................................................

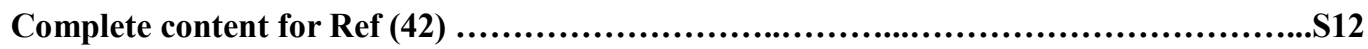

Gibbs Free Energy Corrections and Electronic Energies in Solvate of related compounds

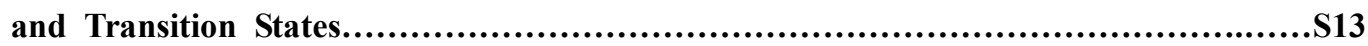

Cartesian Coordinates of Intermediates and Transition States..............................S16 
Optimized structure of Int 15

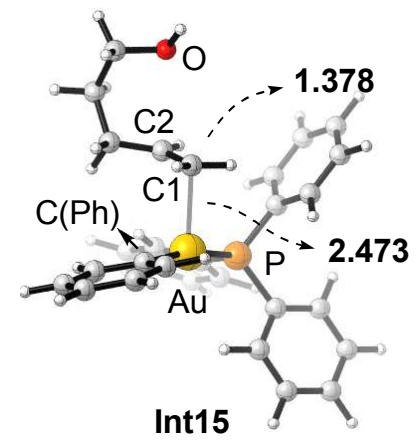

Figure S1. Optimized structures for Int15. Bond lengths are shown in $[\AA]$ 


\section{Complete content for Ref (29)}

The possible proton acceptors in the reaction system are $\mathrm{NTf}_{2}^{-}, \mathrm{BF}_{4}^{-}, \mathrm{PF}_{6}^{-}$and $\mathrm{MeOH}$. In order to examine which one is the most favorable proton receptor, we investigated the following acid-base reactions between Int4 and the above four species (Figure S2). The calculation shows that the reactions are endergonic by $31.8,43.7,54.1$ and $34.3 \mathrm{kcal} / \mathrm{mol}$, respectively. Therefore $\mathrm{NTf}_{2}{ }^{-}$is the most basic and the most possible proton acceptor.

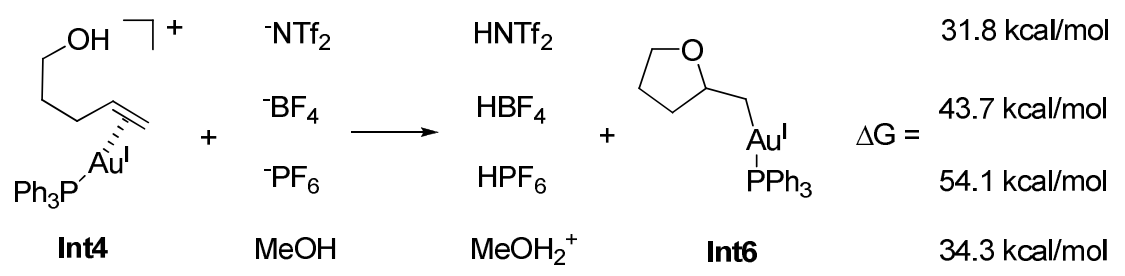

Figure S2 


\section{Complete content for Ref (31)}

The possibility for $\mathrm{Au}(\mathrm{I})$ catalyzed oxidization of $\mathrm{PhN}_{2}{ }^{+}$to phenyl radical is excluded, because the possible transformations are found to be highly endergonic by 33.3 and $36.5 \mathrm{kcal} / \mathrm{mol}$, respectively.

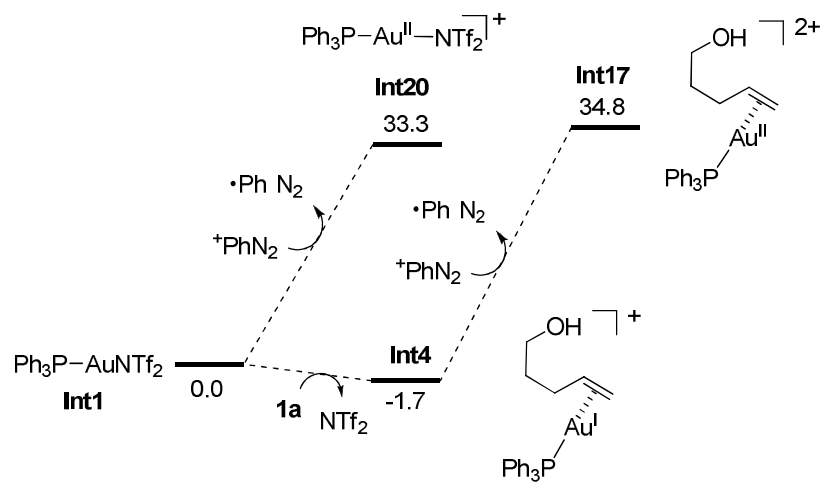

Figure S3 


\section{Complete content for Ref (32)}

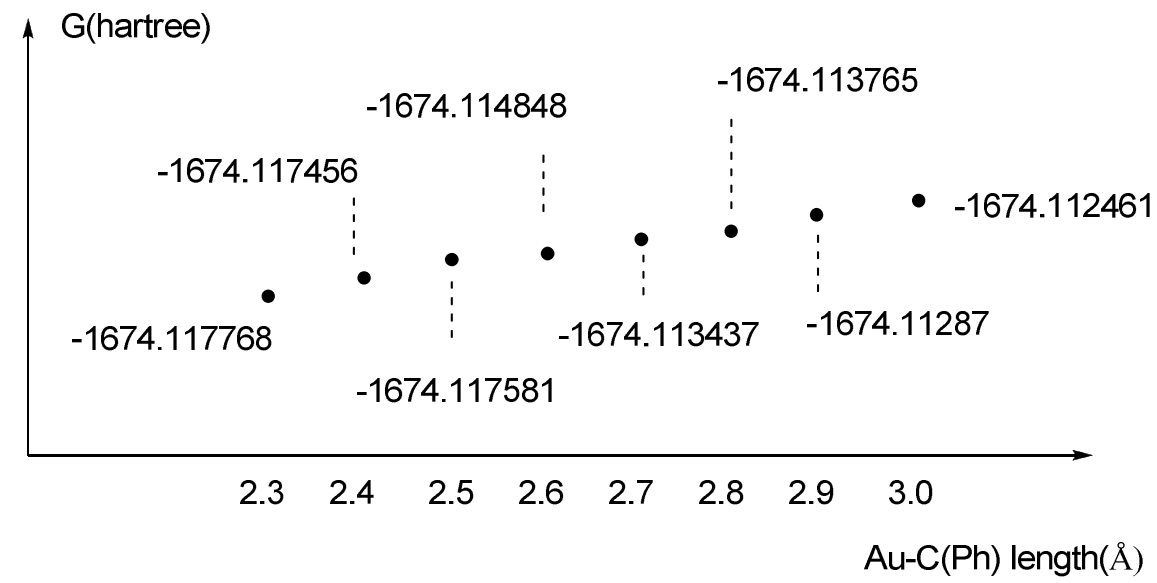

Figure S4

With the decrease of $\mathrm{Au}-\mathrm{C}(\mathrm{Ph})$ bond, the free energy is gradually declining. This phenomenon indicates that there is no transition state in the radical addition process. 


\section{Complete content for Ref (33)}

The energy barrier of the direct SET of Int7 with $\left[\mathrm{Ru}^{\mathrm{III}}\right]$ is $37.1 \mathrm{kcal} / \mathrm{mol}$. Therefore, Int7 first isomerizes to Int8 in which $\mathrm{PPh}_{3}$ and $\mathrm{Ph}$ group are in trans position. Then SET occurs on Int8 with lower energy barrier of $26.8 \mathrm{kcal} / \mathrm{mol}$.

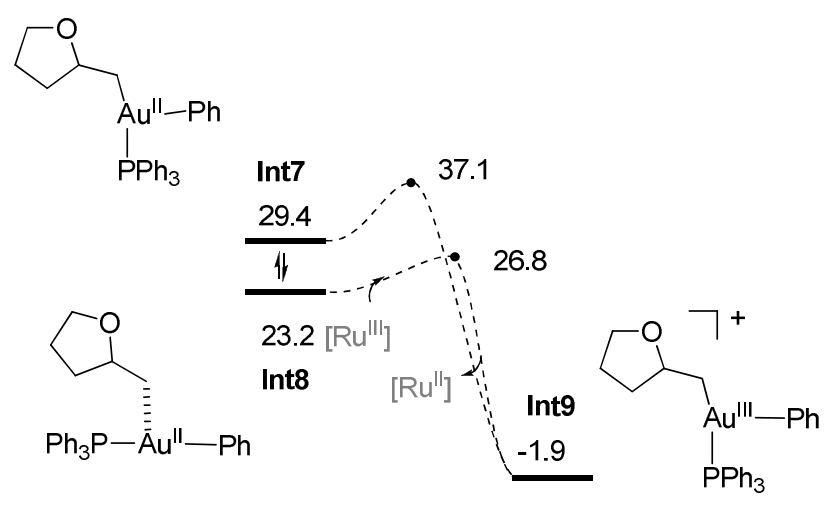

Figure S5 


\section{Complete content for Ref (34)}

According to the Marcus equation, the solvent reorganization energy $\lambda_{0}$ may be calculated from equation (1):

$$
\lambda_{0}=(332 \mathrm{kcal} / \mathrm{mol})\left(\frac{1}{2 a_{1}}+\frac{1}{2 a_{2}}-\frac{1}{R}\right)\left(\frac{1}{\varepsilon_{o p}}-\frac{1}{\varepsilon}\right)
$$

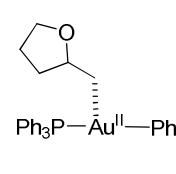

Int8 (23.2)
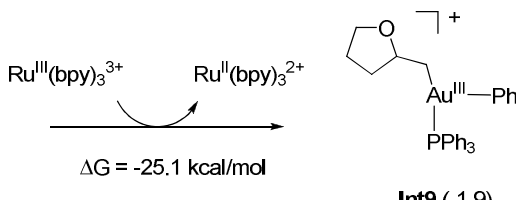

(2)

$a 1$ and $a 2$ are the radii of the molecules Int8 and $\mathrm{Ru}^{\mathrm{III}}(\mathrm{bpy})_{3}{ }^{3+}, R=a_{1}+a_{2}, \xi_{\text {op }}$ is the optical dielectric constant $\left(\xi_{o p}=2.05\right)$, is the static dielectric constant for the $\mathrm{MeOH}$ solvent $(\xi \square=32.6)$. The radii of Int8 and $\mathrm{Ru}{ }^{\mathrm{III}}$ (bpy) ${ }_{3}{ }^{3+}$ are calculated to be 6.27 and $6.37 \AA$. Accordingly. $\lambda_{0}=12.0$ $\mathrm{kcal} / \mathrm{mol}$. We estimate the inner reorganization energy for the reactants $\lambda_{\mathrm{i}}=0$. Thus, the total reorganization energy $\lambda \square=\lambda_{0}+\lambda_{\mathrm{i}}=12.0 \mathrm{kcal} / \mathrm{mol}$. According to Marcus theory, $\Delta G_{r}$ is the reaction energy, and $\Delta G^{\ddagger}$ is the intrinsic barrier.

$$
\Delta G_{E T^{\prime}}^{\ddagger}=\Delta G_{0}^{\ddagger}\left(1+\frac{\Delta G_{r}}{4 \Delta G_{0}^{\ddagger}}\right)^{2} \quad \Delta G_{0}^{\ddagger}=\frac{\lambda}{4}
$$

For eq2, $\Delta G_{r}=-25.1 \mathrm{kcal} / \mathrm{mol}, \Delta G_{0}^{\sharp}=12.0 / 4=3.0 \mathrm{kcal} / \mathrm{mol}$. Therefore, the activation barrier of eq2 is $3.6 \mathrm{kcal} / \mathrm{mol}$.

Besides, the radii of the molecules Int15, Int10, Int4, Int1 are 6.52, 6.38, 5.98 and $5.80 \AA$, respectively. According to the aforementioned method, the barrier for the SET from them are 0.3, $0.1,33.7$ and $28.4 \mathrm{kcal} / \mathrm{mol}$, respectively. 


\section{Complete content for Ref (37)}

$\mathrm{Au}(\mathrm{II})$ intermediates Int15 and Int15' lead to cyclization transition state $\mathbf{T S 5}^{\prime}$ and $\mathbf{T S 5}$, respectively. The more stable intermediate Int15 corresponds to the unstable transition state TS5', however, Int15 could easily isomerize to Int15' with energy barrier of about $5.5 \mathrm{kcal} / \mathbf{m o l}^{\prime}$. Int15' then leads to the stable transition state TS5. Therefore, Int15 $\rightarrow$ Int15 $\rightarrow$ TS5 is the favorable mechanism. Int15' is omitted in the manuscript.

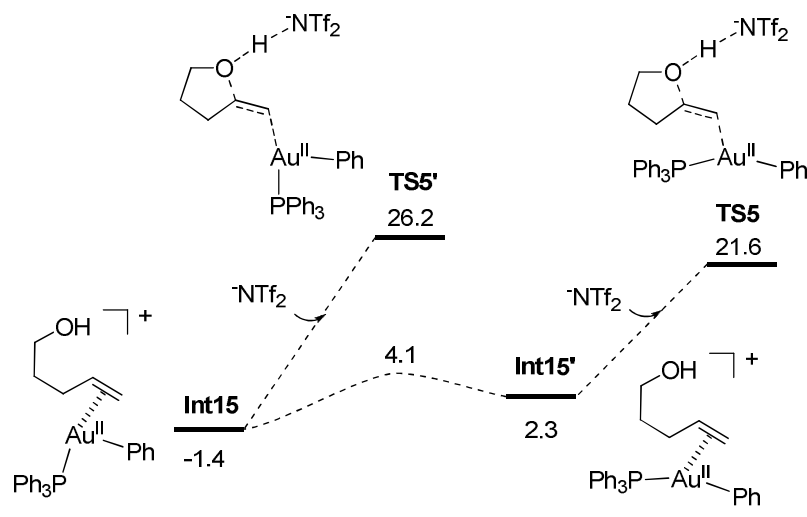

Figure S6 


\section{Complete content for Ref (40)}

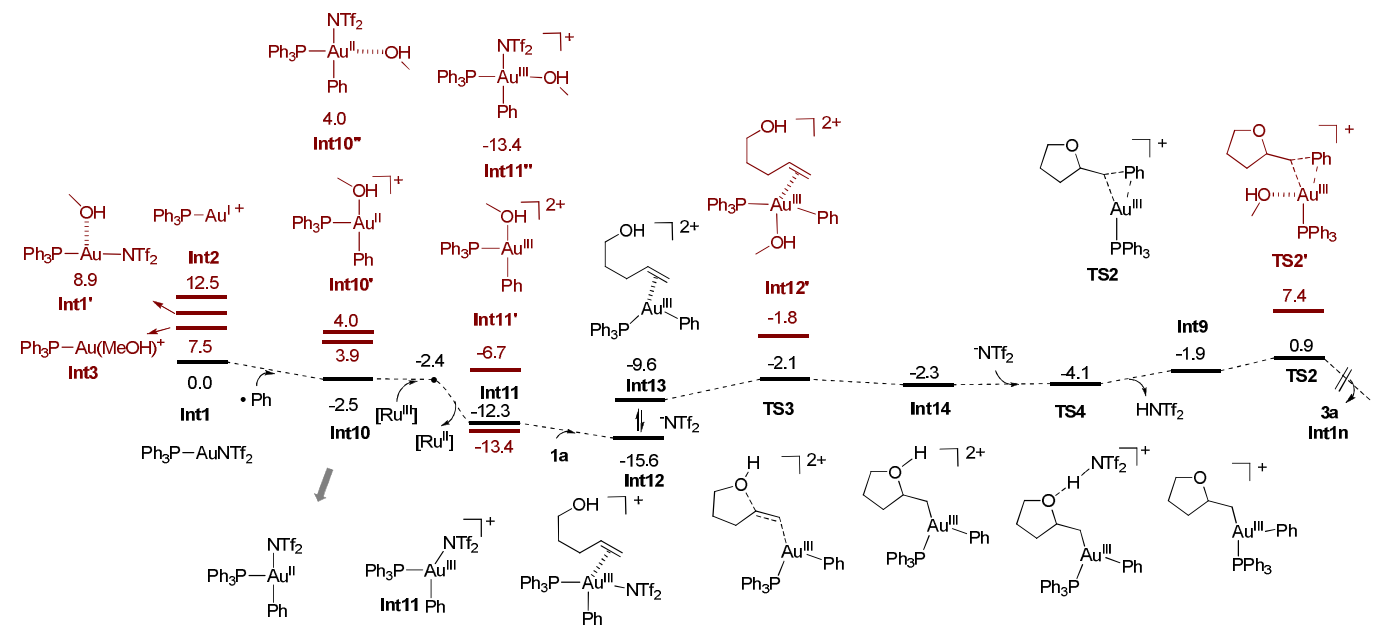

Figure S7

As shown in Figure S7, the free energies of $\left[\mathrm{Ph}_{3} \mathrm{PAu}\right]^{+}, \mathrm{Ph}_{3} \mathrm{PAuNTf}_{2}$ and $\left[\mathrm{Ph}_{3} \mathrm{PAu}(\mathrm{MeOH})\right]^{+}$are $12.5,0.0$ and $7.5 \mathrm{kcal} / \mathrm{mol}$, respectively. Therefore $\mathrm{Ph}_{3} \mathrm{PAuNTf}_{2}$ is the main form of the catalyst, and it was chosen as the energy reference point.

Despite the ligation of $\mathrm{MeOH}$ on the coordination unsaturated $\left[\mathrm{Ph}_{3} \mathrm{PAu}\right]^{+}$is exergonic by 5.0 $\mathrm{kcal} / \mathrm{mol}$ (as expected), the formed $\left[\mathrm{Ph}_{3} \mathrm{PAu}(\mathrm{MeOH})\right]^{+}$is less stable than $\mathrm{NTf}_{2}^{-}$ligated complex $\mathrm{Ph}_{3} \mathrm{PAuNTf}_{2}$ (the reference point in the revised manuscript). Similar observation has also been noted on the intermediate Int10 (i.e. Int10 vs Int10', Figure c14). Meanwhile, an additional $\mathrm{MeOH}$ coordination on Int1 or Int10 is unlikely, because the Au-O bonds in Int1' and Int10" automatically dissociates to $>3.0 \AA$ after geometry optimization. Besides, the free energies of them are relatively higher than Int1 and Int10, respectively. For the subsequent intermediate Int11, the ligand exchange of $\mathrm{NTf}_{2}^{-}$with $\mathrm{MeOH}$ is endergonic by over $5 \mathrm{kcal} / \mathrm{mol}$. The direct addition of $\mathrm{MeOH}$ is exergonic by $1.1 \mathrm{kcal} / \mathrm{mol}$, whereas the ligation of the substrate to form Int12 is more exergonic $(3.3 \mathrm{kcal} / \mathrm{mol})$. Int12 could dissociate $\mathrm{NTf}_{2}^{-}$to form Int13, from which cyclization occurs easily via TS3. Herein, the relative energy of MeOH ligated complex Int12' is even higher than TS3 (as well as all the subsequent species), and therefore $\mathrm{MeOH}$ is unlikely coordinated with the $\mathrm{Au}$ center here. For the reductive elimination transition state, the $\mathrm{Au}-\mathrm{O}$ bonds in the $\mathrm{MeOH}$ coordinated transition state $\mathbf{T S 2}^{\prime}$ also stretch automatically to $>3.0 \AA$ in geometry optimization, and the free energy is higher than TS2 by $6.5 \mathrm{kcal} / \mathrm{mol}$. According to the aforementioned results and discussions, we suggest that $\mathrm{MeOH}$ is unlikely to coordinate with the Au center in our system. 


\section{Regeneration of $\mathrm{NTf}_{2}^{-}$}

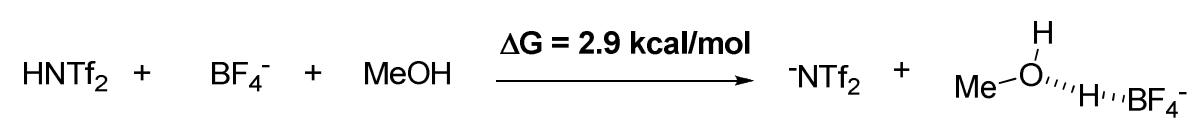

The $\mathrm{NTf}_{2}^{-}$is regenerated by reaction of $\mathrm{HNTf}_{2}$ with $\mathrm{BF}_{4}^{-}$and solvent $(\mathrm{MeOH})$ to give $\mathrm{NTf}_{2}^{-}$and $\mathrm{MeOH}_{2}-\mathrm{BF}_{4}$ complex. This process is sightly endergonic by $2.9 \mathrm{kcal} / \mathrm{mol}$. 


\section{Complete content for Ref (41)}
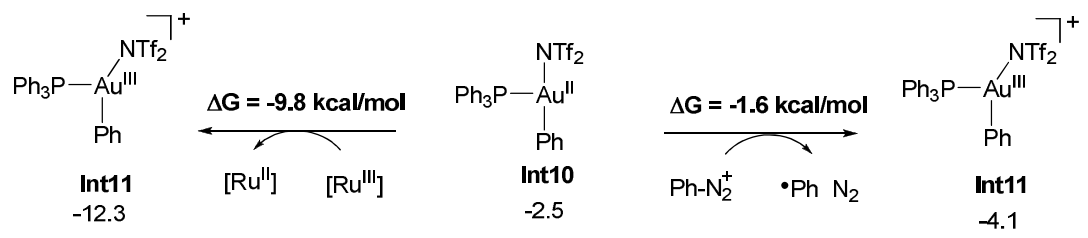

Figure S8

Toste also proposed an alternative mechanism (Toste et al. J. Am. Chem. Soc. 2014, 136, 5844.) that $\mathrm{Au}(\mathrm{II})$ complex reacts with aryldiazonium salt to give $\mathrm{Au}(\mathrm{III})$ complex and a phenyl radical, without regeneration of the photocatalyst.

According to our calculation results, in the favorable mechanisms Path 2A, the SET reaction between $\mathrm{Au}(\mathrm{II})$ complexes Int10 and $\left[\mathrm{Ru}^{\mathrm{III}}\right]$ complex is exergonic by $-9.8 \mathrm{kcal} / \mathrm{mol}$. However, the SET with $\mathrm{PhN}_{2}{ }^{+}$is only exergonic by $-1.6 \mathrm{kcal} / \mathrm{mol}$. The former is more favorable. Therefore, in our concerned reaction system, phenyl radical is generated from photoredox cycle. 


\section{Complete content for Ref (42)}

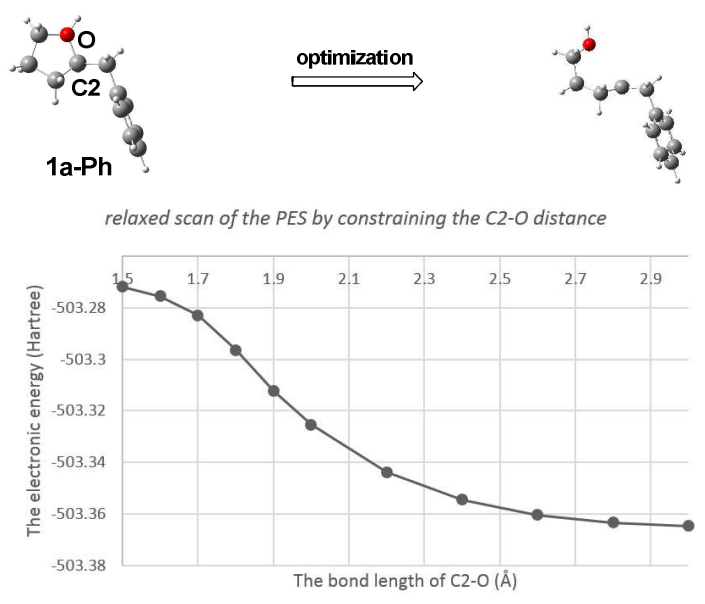

Figure S9

Glorius proposed an alternative mechanism that Meerwein-type addition of phenyl radical to the alkene without Au catalyst could also be feasible. We tried to locate the cyclized intermediate 1a-Ph. However, during geometry optimization, the $\mathrm{C} 2-\mathrm{O}$ bond always break. The structure turns out to be linear. We performed the relaxed scan of the PES by constraining the C2-O distance from $3.0 \AA$ to $1.5 \AA$. As shown in Figure S4, the energy increases during the shortening of $\mathrm{C} 2$-O bond. The overall energy increase is about $58 \mathrm{kcal} / \mathrm{mol}$. Therefore, as proposed by Glorius et al., nonactivated aliphatic alkenes are reluctant for radical addition. 
Gibbs Free Energy Corrections and Electronic Energies in Solvate of related compounds and Transition States

\begin{tabular}{|c|c|c|}
\hline & $\begin{array}{c}\text { Thermal Correction of Gibbs } \\
\text { Free Energies (Hartree) }\end{array}$ & $\begin{array}{c}\text { Electronic Energy in solvent } \\
\text { (Hartree) }\end{array}$ \\
\hline $1 \mathrm{a}$ & 0.111015 & -271.6359084 \\
\hline $2 \mathrm{a}$ & 0.069158 & -340.7594672 \\
\hline $\mathrm{Ph} \bullet$ & 0.059656 & -231.4338832 \\
\hline $3 a$ & 0.190043 & -502.5835071 \\
\hline$-\mathrm{NTf}_{2}$ & 0.009356 & -1827.223398 \\
\hline $\mathrm{HNTf}_{2}$ & 0.019427 & -1827.639773 \\
\hline Int2 & 0.226001 & -1171.561523 \\
\hline Int 3 & 0.274635 & -1287.286778 \\
\hline Int1 & 0.254888 & -2998.824437 \\
\hline Int4 & 0.35381 & -1443.236858 \\
\hline TS1 & 0.387962 & -3270.439677 \\
\hline Int5 & 0.389474 & -3270.438453 \\
\hline Int6 & 0.349874 & -1442.775908 \\
\hline Int7 & 0.428364 & -1674.229822 \\
\hline Int8 & 0.423346 & -1674.234708 \\
\hline Int16 & 0.467325 & -3501.891729 \\
\hline$\left[\mathrm{Ru}^{\mathrm{III}}\right]$ & 0.428685 & -1580.003398 \\
\hline$\left[\mathrm{Ru}^{\mathrm{II}}\right]$ & 0.426745 & -1580.196533 \\
\hline$\left[\mathrm{Ru} \mathrm{u}^{\mathrm{I}}\right]$ & 0.420679 & -1580.303781 \\
\hline
\end{tabular}




\begin{tabular}{|c|c|c|}
\hline$*\left[\mathrm{Ru}^{\mathrm{II}}\right]$ & 0.419674 & -1580.129751 \\
\hline Int9 & 0.433164 & -1674.089316 \\
\hline $\mathrm{TS} 2$ & 0.435259 & -1674.087033 \\
\hline Int10 & 0.338713 & -3230.286438 \\
\hline Int11 & 0.345326 & -3230.113577 \\
\hline Int12 & 0.47857 & -3501.777015 \\
\hline Int13 & 0.443491 & -1674.518296 \\
\hline Int14 & 0.449452 & -1674.512568 \\
\hline Int15 & 0.436474 & -1674.693336 \\
\hline TS5 & 0.466024 & -3501.900129 \\
\hline TS3 & 0.447096 & -1674.51 \\
\hline TS4 & 0.4723 & -3501.752377 \\
\hline TS6 & 0.387547 & -3270.230713 \\
\hline Int17 & 0.35639 & -1442.999047 \\
\hline Int 18 & 0.389045 & -3270.255279 \\
\hline Int19 & 0.346705 & -1442.572189 \\
\hline Int20 & 0.225231 & -1171.32603 \\
\hline $\mathrm{MeOH}$ & 0.028645 & -115.6972548 \\
\hline $\mathrm{MeOH}_{2}-\mathrm{BF}_{4}$ & 0.044997 & -540.748391 \\
\hline $\mathrm{MeOH}_{2}^{+}$ & 0.04244 & -116.1134403 \\
\hline $\mathrm{BF}_{4}^{-}$ & -0.011033 & -424.6221049 \\
\hline $\mathrm{PF}_{6}^{-}$ & -0.008383 & -940.7571295 \\
\hline
\end{tabular}




\begin{tabular}{|c|c|c|}
\hline $\mathrm{HBF}_{4}$ & -0.00693 & -425.013642 \\
\hline $\mathrm{HPF}_{6}$ & -0.004489 & -941.1318499 \\
\hline Int15' & 0.437992 & -1674.688939 \\
\hline TS5' & 0.465776 & -3501.892564 \\
\hline Int1' & 0.303395 & -3114.52734 \\
\hline Int10' & 0.356489 & -1518.748572 \\
\hline Int10" & 0.385613 & -3345.991616 \\
\hline Int11' & 0.360319 & -1518.57415 \\
\hline Int11" & 0.396883 & -3345.835535 \\
\hline Int12' & 0.502167 & -1790.23321 \\
\hline $\mathrm{TS} 2^{\prime}$ & 0.482092 & -1789.792064 \\
\hline
\end{tabular}




\begin{tabular}{|c|c|c|c|c|c|}
\hline \multicolumn{6}{|c|}{ Cartesian Coordinates of Intermediates and Transition States } \\
\hline \multicolumn{3}{|l|}{$1 \mathrm{a}$} & \multirow{2}{*}{$\begin{array}{l}\text { Н } 2.15662800 \\
\text { Н } 0.00003000\end{array}$} & \multirow{2}{*}{$\begin{array}{l}1.17864400 \\
2.41216500\end{array}$} & \multirow{2}{*}{$\begin{array}{r}0.00001100 \\
-0.00002200\end{array}$} \\
\hline C 0.50264600 & -0.34052200 & 0.04789000 & & & \\
\hline C- 0.72813200 & 0.56130600 & 0.25552100 & & & \\
\hline H 0.57153600 & -1.07185800 & 0.86494400 & $3 \mathrm{a}$ & & \\
\hline Н 0.39285900 & -0.91073900 & -0.88415100 & C-1.35509400 & -0.47622100 & 0.12989800 \\
\hline C-2.00615100 & -0.22365300 & 0.37608800 & O-2.61327100 & -1.00177600 & -0.36001900 \\
\hline $\mathrm{H}-0.57823400$ & 1.14874800 & 1.17469100 & C-3.66643300 & -0.04124000 & -0.15667900 \\
\hline H- 0.80923700 & 1.27930500 & -0.57123000 & C-3.12455600 & 0.96318800 & 0.85934400 \\
\hline C-3.06194600 & -0.10989200 & -0.43326000 & C-1.63289500 & 0.98822000 & 0.49941300 \\
\hline H-2.04266300 & -0.94317700 & 1.19699000 & H-1.08463500 & -1.04580500 & 1.03156500 \\
\hline H-3.95797700 & -0.71112800 & -0.29275200 & H-3.90725600 & 0.44767500 & -1.11233300 \\
\hline H-3.07268100 & 0.59261100 & -1.26592100 & H-4.55892800 & -0.57601800 & 0.18747200 \\
\hline C 1.79933100 & 0.45533000 & -0.00718400 & H-3.60797900 & 1.94210700 & 0.78244800 \\
\hline H 1.93111300 & 1.02683600 & 0.92487100 & H-3.26837500 & 0.58813100 & 1.87978700 \\
\hline Н 1.76577300 & 1.18031900 & -0.83513100 & H-1.46425900 & 1.63667700 & -0.36971500 \\
\hline O 2.88287000 & -0.46294300 & -0.18890900 & H-0.99479800 & 1.34001000 & 1.31500100 \\
\hline \multirow[t]{2}{*}{ Н 3.70207200} & 0.05721000 & -0.23536900 & C- 0.27926600 & -0.70421200 & -0.94398100 \\
\hline & & & H- 0.30746600 & -1.76497200 & -1.22398700 \\
\hline $2 \mathrm{a}$ & & & H- 0.55056300 & -0.12428600 & -1.83451100 \\
\hline C-1.48052100 & 1.22248800 & -0.00006300 & C 1.11344100 & -0.33416900 & -0.47952100 \\
\hline C- 0.09444900 & 1.24540100 & 0.00032600 & C 1.69824300 & 0.88950300 & -0.83832600 \\
\hline C 0.56366300 & -0.00000300 & 0.00042600 & C 1.84583900 & -1.20490100 & 0.34350600 \\
\hline C- 0.09445300 & -1.24540400 & 0.00032600 & C 2.97470100 & 1.23640400 & -0.38687200 \\
\hline C-1.48052600 & -1.22248400 & -0.00006300 & H 1.15204000 & 1.57408800 & -1.48353000 \\
\hline C-2.16653900 & 0.00000300 & -0.00035300 & C 3.12123300 & -0.86217300 & 0.79754200 \\
\hline H-2.02912800 & 2.15818400 & -0.00013900 & H 1.41471700 & -2.16377600 & 0.62420100 \\
\hline Н 0.47240300 & 2.16921800 & 0.00058800 & C 3.69073000 & 0.36180700 & 0.43417500 \\
\hline Н 0.47239500 & -2.16922300 & 0.00058800 & Н 3.41024700 & 2.18840800 & -0.68072500 \\
\hline H-2.02913600 & -2.15817800 & -0.00013900 & Н 3.67264300 & -1.55364300 & 1.43019700 \\
\hline H-3.25231400 & 0.00000500 & -0.00096800 & H 4.68511900 & 0.62836400 & 0.78328500 \\
\hline N 1.93510900 & -0.00000300 & 0.00003800 & & & \\
\hline \multirow[t]{2}{*}{ N 3.04813900} & 0.00000200 & -0.00054200 & -NTf2 & & \\
\hline & & & N 0.00002600 & 0.00003500 & 0.86298000 \\
\hline $\mathrm{Ph}$ & & & S 1.15150600 & 0.83865000 & 0.09733300 \\
\hline C-1.21556100 & 0.63305900 & -0.00000600 & O 0.85762500 & 1.23694400 & -1.28968600 \\
\hline C-1.22840200 & -0.77306100 & 0.00002200 & O 1.65509300 & 1.85923200 & 1.02787000 \\
\hline C- 0.00001200 & -1.39915200 & -0.00000900 & C 2.55091200 & -0.38743900 & -0.04479500 \\
\hline C 1.22840400 & -0.77305200 & -0.00000800 & F 2.15187700 & -1.46764500 & -0.72290000 \\
\hline C 1.21557500 & 0.63303100 & 0.00001800 & F 2.96006000 & -0.75837300 & 1.17055300 \\
\hline C- 0.00000300 & 1.32528400 & -0.00000400 & F 3.56882400 & 0.18685200 & -0.69423200 \\
\hline H-2.15663600 & 1.17863400 & -0.00002600 & S-1.15150500 & -0.83864500 & 0.09747900 \\
\hline H-2.16631700 & -1.32302600 & -0.00000900 & O-0.85770700 & -1.23707800 & -1.28951700 \\
\hline Н 2.16628700 & -1.32307400 & -0.00002500 & O-1.65504200 & -1.85913000 & 1.02815000 \\
\hline
\end{tabular}




\begin{tabular}{|c|c|c|c|c|c|}
\hline C-2.55091000 & 0.38744000 & -0.04468300 & H $\quad 1.06886800$ & 1.89679600 & 2.20600200 \\
\hline F-2.15194100 & 1.46752400 & -0.72301700 & H $\quad 0.33679200$ & 4.14583900 & 2.91387400 \\
\hline F-2.95990300 & 0.75857900 & 1.17065500 & H - 1.60656700 & 5.23558600 & 1.81051100 \\
\hline \multirow[t]{2}{*}{ F-3.56891300 } & -0.18694700 & -0.69389500 & H - 2.82086400 & 4.05722000 & -0.01323100 \\
\hline & & & H -2.08739900 & 1.80573700 & -0.73939600 \\
\hline HNTf2 & & & H - 1.60638700 & 0.17464100 & 2.64255000 \\
\hline $\mathrm{N}-0.00017000$ & -0.00051300 & -0.83823000 & H -2.98428300 & -1.49786500 & 3.82591600 \\
\hline Н 0.00046100 & 0.00047600 & -1.86367500 & H -3.20971400 & -3.80217400 & 2.92008500 \\
\hline S 1.24263700 & -0.88063200 & -0.09741800 & H - 2.04289100 & -4.42828600 & 0.81399300 \\
\hline O 0.81841800 & -1.26817000 & 1.23834900 & H -0.67001800 & -2.75543700 & -0.38860800 \\
\hline O 1.70999900 & -1.83209000 & -1.09541800 & H $\quad 2.55590600$ & 0.68611100 & -1.08692500 \\
\hline C 2.58392700 & 0.41828300 & 0.09338600 & H $\quad 4.91373300$ & 0.38737800 & -0.39285100 \\
\hline F 2.13975000 & 1.39421500 & 0.87426200 & H $\quad 5.44623500$ & -0.74481700 & 1.75787000 \\
\hline F 2.89713900 & 0.89145700 & -1.10605500 & H $\quad 3.60830400$ & -1.56680300 & 3.21620100 \\
\hline F 3.63659800 & -0.17428000 & 0.64526600 & H $\quad 1.25313100$ & -1.26429500 & 2.53921200 \\
\hline S-1.24250300 & 0.88049900 & -0.09738800 & & & \\
\hline O-0.81818900 & 1.26795100 & 1.23836100 & Int3 & & \\
\hline O-1.70945300 & 1.83204900 & -1.09551500 & $\mathrm{Au} 1.73774800$ & -0.31771200 & 0.04784900 \\
\hline C-2.58414100 & -0.41810900 & 0.09338000 & P - 0.51666100 & 0.02107900 & -0.00319000 \\
\hline F-2.14008000 & -1.39420600 & 0.87411100 & C -1.45060400 & -1.51222600 & 0.35112600 \\
\hline F-2.89746700 & -0.89104300 & -1.10613100 & C -2.68011700 & -1.78300500 & -0.26905500 \\
\hline \multirow[t]{2}{*}{ F-3.63664300 } & 0.17455500 & 0.64536800 & C -3.37775400 & -2.95080900 & 0.04735900 \\
\hline & & & C - -2.85609200 & -3.85093200 & 0.97930900 \\
\hline Int2 & & & C -1.63128300 & -3.58512500 & 1.59819200 \\
\hline $\mathrm{Au}-0.20443800$ & -0.21984000 & -2.08253000 & C -0.92698900 & -2.42268100 & 1.28389800 \\
\hline $\mathrm{P} \quad 0.01800000$ & 0.01654900 & 0.16600200 & C -1.03562400 & 1.26977100 & 1.23048400 \\
\hline C -0.46742500 & 1.69634100 & 0.69018300 & C -2.26055000 & 1.17077900 & 1.90799900 \\
\hline C $\quad 0.21779400$ & 2.36149800 & 1.71857800 & C -2.63056100 & 2.15592700 & 2.82666400 \\
\hline C -0.19769900 & 3.63327600 & 2.11892700 & C -1.78585300 & 3.24032600 & 3.07329600 \\
\hline C - 1.29022200 & 4.24380900 & 1.49919900 & C -0.56482100 & 3.34252100 & 2.40021100 \\
\hline C -1.97354400 & 3.58287400 & 0.47393600 & C -0.18713400 & 2.36033700 & 1.48471000 \\
\hline C -1.56328200 & 2.31430700 & 0.06581500 & $\mathrm{C}-1.05887300$ & 0.62152500 & -1.64515400 \\
\hline C -1.03874100 & -1.18119000 & 1.04983200 & C -0.41528700 & 0.12463500 & -2.79093100 \\
\hline C - 1.69682400 & -0.82846200 & 2.23807300 & C -0.82078800 & 0.54283400 & -4.05843800 \\
\hline C -2.47454100 & -1.77569300 & 2.90759800 & C -1.86270800 & 1.46531300 & -4.19141000 \\
\hline C -2.59923000 & -3.07008300 & 2.39854600 & C -2.50191700 & 1.96543800 & -3.05483600 \\
\hline C -1.94405200 & -3.42344500 & 1.21495200 & C -2.10481300 & 1.54670100 & -1.78278500 \\
\hline C -1.16862600 & -2.48283600 & 0.53805500 & H -3.09435400 & -1.09101700 & -0.99559300 \\
\hline C $\quad 1.74665400$ & -0.26105700 & 0.68190500 & H -4.32793800 & -3.15618500 & -0.43801400 \\
\hline C $\quad 2.78621800$ & 0.20127500 & -0.14166700 & H -3.40066800 & -4.75994500 & 1.22016300 \\
\hline C $\quad 4.11355700$ & 0.03020700 & 0.24938700 & H - 1.21987900 & -4.28518900 & 2.32009400 \\
\hline C $\quad 4.41105700$ & -0.60644200 & 1.45805100 & H $\quad 0.02970700$ & -2.22286300 & 1.75997500 \\
\hline C $\quad 3.37941900$ & -1.06951100 & 2.27774500 & H - 2.92428300 & 0.33150600 & 1.72537800 \\
\hline C $\quad 2.04710700$ & -0.89995000 & 1.89499000 & H - 3.57848900 & 2.07134600 & 3.35090100 \\
\hline
\end{tabular}




\begin{tabular}{|c|c|c|c|c|c|c|}
\hline \multicolumn{2}{|c|}{ H - 2.07543100} & \multirow{2}{*}{$\begin{array}{r}4.00215300 \\
4.18231100\end{array}$} & \multirow{2}{*}{$\begin{array}{r}3.79199300 \\
2.59291700\end{array}$} & \multirow{2}{*}{$\begin{array}{ll}\mathrm{H} & 2.07093200 \\
\mathrm{H} & 1.19243100\end{array}$} & \multirow{2}{*}{$\begin{array}{l}3.17519700 \\
1.28899600\end{array}$} & \multirow{2}{*}{$\begin{array}{l}3.73737600 \\
2.39716400\end{array}$} \\
\hline $\mathrm{H}$ & 0.09711900 & & & & & \\
\hline $\mathrm{H}$ & 0.76747900 & 2.43705000 & 0.97046000 & H $\quad 1.24067600$ & -2.85391500 & 0.01179700 \\
\hline $\mathrm{H}$ & 0.40306000 & -0.58379800 & -2.68949400 & H 2.14160900 & -4.95564200 & 0.95710000 \\
\hline & -0.31807400 & 0.15458800 & -4.93995100 & $\mathrm{H} \quad 4.24503100$ & -4.92575000 & 2.28640400 \\
\hline & -2.17266600 & 1.79642500 & -5.17888600 & H $\quad 5.44632700$ & -2.78010800 & 2.65673600 \\
\hline & -3.30995800 & 2.68505100 & -3.15455200 & H $\quad 4.56215200$ & -0.67985800 & 1.70846200 \\
\hline & -2.60781300 & 1.94358100 & -0.90637100 & $\mathrm{~N}-2.31553100$ & -0.04161400 & 0.01758400 \\
\hline & 4.78383500 & 0.51399200 & 0.05114600 & S -3.14577200 & -1.41896900 & -0.38401100 \\
\hline $\mathrm{H}$ & 4.53691600 & 1.11561000 & 0.92616000 & O -4.47708000 & -1.16500700 & -0.93674300 \\
\hline $\mathrm{H}$ & 5.82202100 & 0.17593300 & 0.11007600 & O - -2.21153600 & -2.29732700 & -1.09302600 \\
\hline $\mathrm{H}$ & 4.62216200 & 1.09362100 & -0.86204900 & C -3.40848900 & -2.22743000 & 1.28322300 \\
\hline $\mathrm{O}$ & 3.91305400 & -0.64755900 & 0.08618600 & F - 4.10510800 & -1.41615800 & 2.07703700 \\
\hline \multirow[t]{2}{*}{$\mathrm{H}$} & 4.12206600 & -1.21856000 & -0.67563800 & F - -2.22487500 & -2.48991300 & 1.83867500 \\
\hline & & & & F - 4.07951000 & -3.36377200 & 1.10008700 \\
\hline \multicolumn{2}{|c|}{ Int1 } & & & S -3.07010800 & 1.39978400 & 0.33542000 \\
\hline \multicolumn{2}{|c|}{$\mathrm{Au}-0.14002300$} & -0.09788100 & 0.04092800 & $\mathrm{O}-4.47829100$ & 1.25310000 & 0.70659600 \\
\hline $\mathrm{P}$ & 2.15822900 & -0.10141800 & 0.03729300 & O -2.16757400 & 2.19132300 & 1.17487300 \\
\hline $\mathrm{C}$ & 2.82782100 & 0.01556200 & -1.66304500 & C -3.04750700 & 2.23978700 & -1.33750400 \\
\hline $\mathrm{C}$ & 4.01702800 & -0.62977600 & -2.03504800 & F - -3.68135500 & 1.48334000 & -2.23168500 \\
\hline $\mathrm{C}$ & 4.50457900 & -0.49776000 & -3.33734800 & F - 1.78465500 & 2.42778200 & -1.72554900 \\
\hline C & 3.81301200 & 0.27623000 & -4.27196500 & F - -3.66038600 & 3.41724100 & -1.22766600 \\
\hline C & 2.62821800 & 0.92089300 & -3.90520500 & & & \\
\hline $\mathrm{C}$ & 2.13281300 & 0.78889200 & -2.60786500 & Int4 & & \\
\hline$C$ & 2.82633600 & 1.31928100 & 0.97913800 & C -3.45383100 & -0.31277500 & 0.37107400 \\
\hline $\mathrm{C}$ & 4.02030300 & 1.95574600 & 0.60577900 & O -6.34838900 & -0.87716000 & 0.39879400 \\
\hline $\mathrm{C}$ & 4.50726200 & 3.02427200 & 1.36192500 & C -6.48274100 & 0.35672900 & -0.31403400 \\
\hline $\mathrm{C}$ & 3.81010900 & 3.46277900 & 2.49013100 & C -5.39730100 & 1.31845600 & 0.14770300 \\
\hline $\mathrm{C}$ & 2.61968300 & 2.83310800 & 2.86425700 & C -3.96018700 & 0.94365600 & -0.28158200 \\
\hline $\mathrm{C}$ & 2.12511700 & 1.76830100 & 2.11060200 & H -3.47165400 & -0.32758800 & 1.46131600 \\
\hline $\mathrm{C}$ & 2.84456300 & -1.62442900 & 0.78573400 & H -6.40362000 & 0.18793200 & -1.39857000 \\
\hline $\mathrm{C}$ & 2.16554200 & -2.83740700 & 0.58287500 & $\mathrm{H}-7.47037300$ & 0.80221400 & -0.12059300 \\
\hline $\mathrm{C}$ & 2.67309800 & -4.02164300 & 1.11766200 & H -5.62114300 & 2.30740200 & -0.27019600 \\
\hline $\mathrm{C}$ & 3.85451000 & -4.00355500 & 1.86460900 & H -5.44768400 & 1.41622300 & 1.24030900 \\
\hline $\mathrm{C}$ & 4.52961000 & -2.79893700 & 2.07370500 & H -3.90557400 & 0.84828100 & -1.37280800 \\
\hline $\mathrm{C}$ & 4.02950700 & -1.61025400 & 1.53717200 & H -3.30613000 & 1.77617400 & 0.00818500 \\
\hline $\mathrm{H}$ & 4.56273900 & -1.23419900 & -1.31713300 & C -3.09597300 & -1.46386500 & -0.28510000 \\
\hline $\mathrm{H}$ & 5.42400500 & -1.00329600 & -3.61962900 & H -2.92873000 & -2.38558900 & 0.26913100 \\
\hline $\mathrm{H}$ & 4.19308200 & 0.37336700 & -5.28536500 & H -3.20731900 & -1.54719000 & -1.36488700 \\
\hline $\mathrm{H}$ & 2.08444400 & 1.52003900 & -4.63041400 & $\mathrm{Au}-1.03629700$ & -0.48238800 & -0.01656200 \\
\hline $\mathrm{H}$ & 1.20531300 & 1.28299800 & -2.32962500 & $\mathrm{P} \quad 1.25049700$ & 0.04891500 & 0.00919000 \\
\hline $\mathrm{H}$ & 4.56854700 & 1.62543000 & -0.27111000 & C $\quad 1.93582700$ & 0.02469400 & 1.70607600 \\
\hline $\mathrm{H}$ & 5.43017600 & 3.51482500 & 1.06500800 & C 2.98995000 & 0.86896600 & 2.08873400 \\
\hline $\mathrm{H}$ & 4.19030500 & 4.29715500 & 3.07330900 & C 3.49872800 & 0.80455600 & 3.38765700 \\
\hline
\end{tabular}




\begin{tabular}{|c|c|c|c|c|c|c|c|}
\hline $\mathrm{C}$ & 2.96232400 & -0.09800500 & 4.30911500 & $\mathrm{H}$ & 0.17706300 & -0.90722100 & 3.24692900 \\
\hline $\mathrm{C}$ & 1.91185600 & -0.93960100 & 3.93268900 & & 0.81597300 & -0.13012000 & 0.42324600 \\
\hline $\mathrm{C}$ & 1.39563400 & -0.87693800 & 2.63792300 & & -1.24853200 & -0.51065700 & -0.51125800 \\
\hline C & 2.21132900 & -1.15081400 & -0.98555100 & & -1.20343000 & 0.88297700 & 0.58699100 \\
\hline $\mathrm{C}$ & 3.49744800 & -1.56596900 & -0.60875200 & & 1.26770900 & -0.02755800 & 0.14684700 \\
\hline $\mathrm{C}$ & 4.20064200 & -2.46998000 & -1.40851000 & $\mathrm{P}$ & 3.64365500 & 0.08894300 & -0.19240900 \\
\hline $\mathrm{C}$ & 3.62896000 & -2.96137100 & -2.58423000 & $\mathrm{C}$ & 4.31474400 & 1.79224700 & -0.03963700 \\
\hline $\mathrm{C}$ & 2.34774700 & -2.55023600 & -2.96320600 & $\mathrm{C}$ & 5.40208800 & 2.24848900 & -0.80097300 \\
\hline $\mathrm{C}$ & 1.63768800 & -1.65224800 & -2.16609800 & $\mathrm{C}$ & 5.87683300 & 3.55188900 & -0.63601100 \\
\hline $\mathrm{C}$ & 1.58473200 & 1.71061400 & -0.68407300 & $\mathrm{C}$ & 5.27341200 & 4.40857500 & 0.28793200 \\
\hline $\mathrm{C}$ & 0.68301900 & 2.74909200 & -0.39748900 & $\mathrm{C}$ & 4.18893300 & 3.96144800 & 1.04775000 \\
\hline 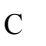 & 0.91394500 & 4.03356200 & -0.88971400 & $\mathrm{C}$ & 3.70744200 & 2.66202700 & 0.88192200 \\
\hline $\mathrm{C}$ & 2.03880300 & 4.28881800 & -1.67972500 & $\mathrm{C}$ & 4.58135800 & -0.93058400 & 1.01535900 \\
\hline $\mathrm{C}$ & 2.93457800 & 3.25812100 & -1.97237700 & $\mathrm{C}$ & 5.81208700 & -0.52530600 & 1.55352900 \\
\hline $\mathrm{C}$ & 2.71276800 & 1.97100600 & -1.47657200 & $\mathrm{C}$ & 6.48212900 & -1.34199700 & 2.46804600 \\
\hline $\mathrm{H}$ & 3.41386300 & 1.57506300 & 1.38126800 & $\mathrm{C}$ & 5.93260200 & -2.56779800 & 2.85021200 \\
\hline $\mathrm{H}$ & 4.31324900 & 1.46250600 & 3.67787500 & $\mathrm{C}$ & 4.70688600 & -2.97797400 & 2.31765000 \\
\hline $\mathrm{H}$ & 3.35834200 & -0.14219100 & 5.32007000 & $\mathrm{C}$ & 4.03062300 & -2.16210800 & 1.40976900 \\
\hline $\mathrm{H}$ & 1.48837100 & -1.63921500 & 4.64808300 & $\mathrm{C}$ & 4.15572300 & -0.50404100 & -1.85472100 \\
\hline П & 0.57055800 & -1.52473700 & 2.35175500 & $\mathrm{C}$ & 3.31216300 & -0.22429700 & -2.94345700 \\
\hline $\mathrm{H}$ & 3.95271700 & -1.19104000 & 0.30255200 & $\mathrm{C}$ & 3.66150700 & -0.63668800 & -4.23001700 \\
\hline $\mathrm{H}$ & 5.19514900 & -2.78965100 & -1.10936100 & $\mathrm{C}$ & 4.84946900 & -1.34238900 & -4.44065300 \\
\hline $\mathrm{H}$ & 4.17792400 & -3.66691900 & -3.20206700 & $\mathrm{C}$ & 5.68889700 & -1.63061800 & -3.36199600 \\
\hline 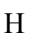 & 1.89733300 & -2.93385700 & -3.87455300 & $\mathrm{C}$ & 5.34650100 & -1.21364100 & -2.07326500 \\
\hline $\mathrm{H}$ & 0.63701500 & -1.34218800 & -2.45776300 & $\mathrm{H}$ & 5.87938800 & 1.59259200 & -1.52279800 \\
\hline & -0.19857900 & 2.55071300 & 0.20704400 & $\mathrm{H}$ & 6.71795000 & 3.89652600 & -1.23166100 \\
\hline $\mathrm{H}$ & 0.21229300 & 4.83169600 & -0.66338100 & $\mathrm{H}$ & 5.64320000 & 5.42297700 & 0.41183400 \\
\hline $\mathrm{H}$ & 2.21344800 & 5.28801300 & -2.06951400 & $\mathrm{H}$ & 3.71204800 & 4.62552100 & 1.76370900 \\
\hline $\mathrm{H}$ & 3.80856800 & 3.45228600 & -2.58808500 & $\mathrm{H}$ & 2.85591400 & 2.32190100 & 1.46623100 \\
\hline $\mathrm{H}$ & 3.41606200 & 1.17796800 & -1.71076900 & $\mathrm{H}$ & 6.25036400 & 0.42461600 & 1.26347200 \\
\hline \multirow{2}{*}{\multicolumn{2}{|c|}{ H -6.97262300 }} & -1.51289000 & 0.01141200 & $\mathrm{H}$ & 7.43364700 & -1.01753800 & 2.88094600 \\
\hline & & & & $\mathrm{H}$ & 6.45465100 & -3.20004700 & 3.56357000 \\
\hline \multicolumn{2}{|c|}{ TS1 } & & & $\mathrm{H}$ & 4.27270600 & -3.92891600 & 2.61464900 \\
\hline \multicolumn{2}{|c|}{ C -1.21176400} & -1.02041200 & 1.57287000 & $\mathrm{H}$ & 3.07204500 & -2.48137200 & 1.00768600 \\
\hline \multicolumn{2}{|c|}{ O - -2.72252000} & -1.13000700 & 1.66797700 & $\mathrm{H}$ & 2.38163500 & 0.31515800 & -2.78335100 \\
\hline \multicolumn{2}{|c|}{ C -3.17711400} & -0.96071000 & 3.04380400 & $\mathrm{H}$ & 3.00265800 & -0.41388700 & -5.06507800 \\
\hline \multicolumn{2}{|c|}{ C - 1.91840800} & -1.14090200 & 3.88463100 & $\mathrm{H}$ & 5.11801600 & -1.66942700 & -5.44170300 \\
\hline \multicolumn{2}{|c|}{ C -0.82505600} & -0.55983600 & 2.97924300 & $\mathrm{H}$ & 6.61234000 & -2.18129200 & -3.52065700 \\
\hline \multicolumn{2}{|c|}{ Н - 0.89475700} & -2.05406300 & 1.40327700 & $\mathrm{H}$ & 6.00806300 & -1.44232100 & -1.24322400 \\
\hline \multicolumn{2}{|c|}{ H -3.60357300} & 0.04248500 & 3.14963400 & & .47366700 & -0.54879300 & 0.75561600 \\
\hline \multicolumn{2}{|c|}{ H -3.95358000} & -1.70571600 & 3.23295400 & & .24905900 & -0.19652500 & -0.08160200 \\
\hline \multicolumn{2}{|c|}{ H - 1.99833100} & -0.62946800 & 4.84842700 & & 82999300 & 1.36170400 & -0.03966300 \\
\hline \multicolumn{2}{|c|}{ H - 1.73655400} & -2.20513700 & 4.07380500 & & 23353600 & 1.44043300 & -0.43599300 \\
\hline \multicolumn{2}{|c|}{ H - 0.83087800} & 0.53670700 & 3.02338300 & & -4.35843800 & 1.94032000 & 1.21730800 \\
\hline
\end{tabular}




\begin{tabular}{|c|c|c|c|c|c|}
\hline C -3.86135100 & 2.21488800 & -1.40061800 & C 3.54164100 & -2.19909900 & 3.66071400 \\
\hline F -3.95775100 & 1.51958000 & -2.53004900 & C $\quad 4.69158300$ & -1.80118100 & 4.34833500 \\
\hline F - 2.58464600 & 2.32252300 & -1.04312300 & C $\quad 5.55448000$ & -0.86255900 & 3.77746800 \\
\hline F - 4.38601600 & 3.42694500 & -1.56881800 & C $\quad 5.27384000$ & -0.32233300 & 2.51997900 \\
\hline S - 4.87060800 & -1.42845300 & -1.01662500 & H $\quad 6.00751900$ & -1.97109000 & 0.27041000 \\
\hline O -5.57780200 & -0.92029100 & -2.18811700 & H $\quad 6.97985400$ & -3.48309500 & -1.42302200 \\
\hline O -3.81090200 & -2.42681700 & -1.13469400 & H $\quad 6.00086400$ & -3.60182200 & -3.70618000 \\
\hline C -6.16079200 & -2.19055800 & 0.10806300 & H $\quad 4.03291700$ & -2.19269600 & -4.28463900 \\
\hline F - 7.09582000 & -1.29114800 & 0.39968000 & H $\quad 3.04368800$ & -0.68618300 & -2.58798400 \\
\hline F -5.56991700 & -2.60761000 & 1.22648200 & H $\quad 6.35801100$ & 0.73577500 & -0.98006700 \\
\hline \multirow[t]{2}{*}{ F -6.70691900 } & -3.22446500 & -0.52741700 & H $\quad 7.48259900$ & 2.92564800 & -1.15586900 \\
\hline & & & H $\quad 6.37797100$ & 4.97600800 & -0.28348900 \\
\hline Int5 & & & H 4.12714900 & 4.81773000 & 0.76736600 \\
\hline C -1.22725900 & 1.74773300 & -0.73795000 & H $\quad 2.98528800$ & 2.62917900 & 0.93145800 \\
\hline O - 2.72125300 & 1.80141400 & -0.80434400 & H $\quad 2.35173100$ & -1.95982400 & 1.88152700 \\
\hline C -3.16343700 & 2.51727000 & -1.98616600 & H $\quad 2.86387100$ & -2.92462100 & 4.10256300 \\
\hline C -1.92334700 & 3.24759700 & -2.49562700 & H $\quad 4.91187600$ & -2.21754100 & 5.32772200 \\
\hline C -0.79583700 & 2.28365900 & -2.10638100 & H $\quad 6.44801700$ & -0.54760200 & 4.30991300 \\
\hline H - 0.96143100 & 2.46248700 & 0.05008300 & H $\quad 5.95299200$ & 0.40585200 & 2.08698000 \\
\hline H -3.53914100 & 1.79336100 & -2.71967400 & H -3.55977700 & 0.59517300 & -0.40804500 \\
\hline H -3.98260200 & 3.18187200 & -1.69550800 & $\mathrm{~N}-4.26712100$ & -0.14301600 & -0.00991000 \\
\hline H - 1.97562500 & 3.44465700 & -3.57075600 & S -4.80343900 & -1.33997600 & -1.05143700 \\
\hline H - 1.80524600 & 4.20626700 & -1.97680500 & O -6.19506300 & -1.68584800 & -0.79018700 \\
\hline H -0.73246800 & 1.45577600 & -2.82448100 & $\mathrm{O}-4.33312800$ & -0.95910300 & -2.37968100 \\
\hline H $\quad 0.18426900$ & 2.76744500 & -2.05658200 & C -3.77616900 & -2.81685700 & -0.52108100 \\
\hline C -0.76807400 & 0.35359300 & -0.37445200 & F -3.95872000 & -3.04223700 & 0.77556200 \\
\hline H - 1.22931600 & 0.04950300 & 0.57500100 & F - 2.49277300 & -2.57263300 & -0.76456000 \\
\hline H -1.10012700 & -0.35436400 & -1.14541200 & F - 4.18245100 & -3.86655200 & -1.22943400 \\
\hline $\mathrm{Au} 1.31356200$ & 0.17666800 & -0.13854300 & S - 4.89040600 & 0.21864400 & 1.50336600 \\
\hline P $\quad 3.68900600$ & -0.06403300 & 0.16141100 & O -5.58597900 & -0.93616100 & 2.05657000 \\
\hline C $\quad 4.46252100$ & -1.21489400 & -1.04387200 & O -3.83296700 & 0.91266500 & 2.22960900 \\
\hline C $\quad 5.57199500$ & -2.01279300 & -0.72339100 & C -6.18896500 & 1.51292500 & 1.11043200 \\
\hline C $\quad 6.12221700$ & -2.86757400 & -1.68139000 & F -7.09270600 & 1.00123800 & 0.28134200 \\
\hline C $\quad 5.57260800$ & -2.93303300 & -2.96423500 & F -5.59629600 & 2.56338400 & 0.54934400 \\
\hline C $\quad 4.46764700$ & -2.14186200 & -3.28995100 & F -6.76795200 & 1.87204600 & 2.25208300 \\
\hline C 3.91105900 & -1.29065800 & -2.33405300 & & & \\
\hline C $\quad 4.59541100$ & 1.52636000 & -0.00244600 & Int6 & & \\
\hline C $\quad 5.86414200$ & 1.62227500 & -0.59414900 & C - 4.11298600 & -0.40619500 & 0.20041000 \\
\hline C $\quad 6.50132000$ & 2.86189800 & -0.69311000 & O -5.46196000 & -0.97151400 & 0.07622400 \\
\hline C $\quad 5.88079500$ & 4.01299800 & -0.20245400 & C -6.38157100 & 0.02311500 & -0.40390000 \\
\hline C $\quad 4.61675800$ & 3.92487100 & 0.38779800 & C -5.65935900 & 1.36690600 & -0.28112700 \\
\hline C 3.97378900 & 2.69005100 & 0.48251200 & C -4.20073900 & 0.95305500 & -0.50694400 \\
\hline C 4.12180300 & -0.71858900 & 1.82324400 & H - 3.93743800 & -0.24867400 & 1.27579100 \\
\hline C 3.25401600 & -1.65691500 & 2.40738200 & H -6.64064900 & -0.19205600 & -1.45205400 \\
\hline
\end{tabular}




\begin{tabular}{|c|c|c|c|c|c|c|c|}
\hline \multicolumn{2}{|c|}{ H -7.30396200 } & -0.03058200 & 0.18785800 & \multicolumn{4}{|c|}{ Int7 } \\
\hline \multicolumn{2}{|c|}{ H -6.02483300 } & 2.10687900 & -1.00062300 & \multicolumn{2}{|c|}{ C -3.79968000} & -1.01403700 & -0.53758700 \\
\hline \multicolumn{2}{|c|}{ H -5.78792800 } & 1.77941900 & 0.72725400 & \multicolumn{2}{|c|}{ O -5.22053700 } & -0.89119400 & -0.89497100 \\
\hline \multicolumn{2}{|c|}{ H -3.99876500 } & 0.82803200 & -1.57943900 & \multicolumn{2}{|c|}{ C -6.04506100} & -1.44872100 & 0.14109100 \\
\hline \multicolumn{2}{|c|}{ H -3.47770200 } & 1.67143600 & -0.10756900 & \multicolumn{2}{|c|}{ C -5.12451100} & -2.34054600 & 0.97371700 \\
\hline \multicolumn{2}{|c|}{ C -3.08928500} & -1.38574400 & -0.34875000 & \multicolumn{2}{|c|}{ C -3.80267600} & -1.56739900 & 0.89598800 \\
\hline \multicolumn{2}{|c|}{ H -3.16612800 } & -2.33816400 & 0.19557000 & \multicolumn{2}{|c|}{ H -3.37434100 } & -1.75935700 & -1.22552000 \\
\hline \multicolumn{2}{|c|}{ H -3.32266600 } & -1.60002700 & -1.40254600 & \multicolumn{2}{|c|}{ H -6.47214200} & -0.64056000 & 0.75510200 \\
\hline \multicolumn{2}{|r|}{ Au-1.09823900 } & -0.72467200 & -0.21995000 & \multicolumn{2}{|c|}{ H -6.87458500 } & -1.99104800 & -0.32851900 \\
\hline & 1.19664000 & 0.00777000 & -0.08660100 & \multicolumn{2}{|c|}{ H -5.48916100 } & -2.48511000 & 1.99591900 \\
\hline & 1.67052700 & 1.14452300 & -1.45070900 & \multicolumn{2}{|c|}{ H -5.02197400 } & -3.32639100 & 0.50350500 \\
\hline & 2.96442000 & 1.17811600 & -1.99395300 & \multicolumn{2}{|c|}{ H -3.80284900 } & -0.73733100 & 1.61512100 \\
\hline $\mathrm{C}$ & 3.26866700 & 2.06498000 & -3.02938800 & \multicolumn{2}{|c|}{ H - 2.92332900} & -2.18727300 & 1.09684400 \\
\hline $\mathrm{C}$ & 2.28776200 & 2.92461400 & -3.53017200 & \multicolumn{2}{|c|}{ C -3.10528800 } & 0.32292000 & -0.72901100 \\
\hline 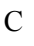 & 0.99701300 & 2.89563000 & -2.99509200 & & 3.21361000 & 0.66567500 & -1.76676000 \\
\hline $\mathrm{C}$ & 0.68713400 & 2.00668600 & -1.96464200 & & .55774700 & 1.07826400 & -0.07468500 \\
\hline $\mathrm{C}$ & 1.56722700 & 0.90864500 & 1.47194300 & & -1.00860700 & 0.23323000 & -0.35533200 \\
\hline $\mathrm{C}$ & 2.41838800 & 2.02277500 & 1.52361900 & $\mathrm{P}$ & 1.32077900 & -0.50953000 & -0.01747100 \\
\hline $\mathrm{C}$ & 2.65860700 & 2.66746600 & 2.73979700 & $C$ & 1.99813200 & -0.04605000 & 1.62727600 \\
\hline $\mathrm{C}$ & 2.05481800 & 2.20629500 & 3.91178500 & 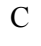 & 3.26731500 & 0.52479400 & 1.79864900 \\
\hline $\mathrm{C}$ & 1.20599800 & 1.09651300 & 3.86759200 & $\mathrm{C}$ & 3.71595400 & 0.87099700 & 3.07676400 \\
\hline $\mathrm{C}$ & 0.95784800 & 0.45389200 & 2.65412800 & C & 2.90611000 & 0.64751700 & 4.19154000 \\
\hline $\mathrm{C}$ & 2.40229300 & -1.37787100 & -0.15797400 & $\mathrm{C}$ & 1.63895400 & 0.07853200 & 4.02822200 \\
\hline C & 2.10605300 & -2.46757000 & -0.99460500 & $\mathrm{C}$ & 1.18361500 & -0.26041900 & 2.75395900 \\
\hline 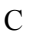 & 2.99457700 & -3.53872900 & -1.09838700 & $c$ & 1.46126600 & -2.34457400 & -0.08896400 \\
\hline $\mathrm{C}$ & 4.18143200 & -3.53808200 & -0.36009800 & $\mathrm{C}$ & 2.34338500 & -3.07162600 & 0.72559400 \\
\hline$c$ & 4.47857700 & -2.46188300 & 0.47939600 & C & 2.41344300 & -4.46321600 & 0.61985000 \\
\hline 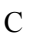 & 3.59504800 & -1.38436700 & 0.58133400 & $C$ & 1.60992100 & -5.14057000 & -0.30019800 \\
\hline $\mathrm{H}$ & 3.73509400 & 0.51375300 & -1.61480000 & $\mathrm{C}$ & 0.73093600 & -4.42300300 & -1.11653200 \\
\hline $\mathrm{H}$ & 4.27276000 & 2.08144900 & -3.44489000 & $\mathrm{C}$ & 0.65196500 & -3.03401200 & -1.00773000 \\
\hline $\mathrm{H}$ & 2.52673000 & 3.61071600 & -4.33845000 & $\mathrm{C}$ & 2.52435600 & 0.10688400 & -1.26410400 \\
\hline $\mathrm{H}$ & 0.22866900 & 3.55815200 & -3.38463300 & $\mathrm{C}$ & 2.28544700 & 1.35124200 & -1.87057300 \\
\hline & -0.32169300 & 1.97791800 & -1.56018300 & $\mathrm{C}$ & 3.17815100 & 1.85692500 & -2.81771400 \\
\hline$\Pi$ & 2.89398700 & 2.39125300 & 0.61985600 & $\mathrm{C}$ & 4.31184200 & 1.12300200 & -3.17500000 \\
\hline $\mathrm{H}$ & 3.31762000 & 3.53126000 & 2.76833000 & $\mathrm{C}$ & 4.55313200 & -0.11821300 & -2.58007700 \\
\hline $\mathrm{H}$ & 2.24118200 & 2.71203300 & 4.85553500 & $\mathrm{C}$ & 3.66551500 & -0.62608300 & -1.62949400 \\
\hline $\mathrm{H}$ & 0.73036300 & 0.73548000 & 4.77558600 & $\mathrm{H}$ & 3.90967900 & 0.70134200 & 0.94170900 \\
\hline $\mathrm{H}$ & 0.28770900 & -0.40195900 & 2.62421900 & $\mathrm{H}$ & 4.70064500 & 1.31497700 & 3.19725900 \\
\hline 11 & 1.17912300 & -2.47769700 & -1.56310800 & $\mathrm{H}$ & 3.25723700 & 0.91819000 & 5.18375800 \\
\hline $\mathrm{H}$ & 2.75573300 & -4.37593100 & -1.74882600 & $\mathrm{H}$ & 1.00276900 & -0.09590000 & 4.89185000 \\
\hline $\mathrm{H}$ & 4.87009000 & -4.37542300 & -0.43559800 & $\mathrm{H}$ & 0.19311500 & -0.69343600 & 2.63428300 \\
\hline $\mathrm{H}$ & 5.39910000 & -2.45855300 & 1.05707400 & $\mathrm{H}$ & 2.97639400 & -2.55858100 & 1.44320300 \\
\hline $\mathrm{H}$ & 3.83777700 & -0.55310200 & 1.23624800 & $\mathrm{H}$ & 3.09757700 & -5.01609500 & 1.25823000 \\
\hline & & & & $\mathrm{H}$ & 1.66481400 & -6.22316700 & -0.37833100 \\
\hline
\end{tabular}




\begin{tabular}{|c|c|c|c|c|c|}
\hline H $\quad 0.10061400$ & -4.94392100 & -1.83240500 & C -2.62776300 & 2.54162100 & -1.22775300 \\
\hline H - 0.04424400 & -2.48197900 & -1.63512500 & C -2.76926400 & 3.39712800 & -2.32315800 \\
\hline H 1.40184700 & 1.92395700 & -1.60304100 & C -2.24355900 & 3.04033100 & -3.56714100 \\
\hline H $\quad 2.98276800$ & 2.82080900 & -3.28028800 & C -1.57093100 & 1.82416000 & -3.71605000 \\
\hline H $\quad 5.00298400$ & 1.51464300 & -3.91675000 & C -1.42151700 & 0.96930500 & -2.62303000 \\
\hline H $\quad 5.43217800$ & -0.69465800 & -2.85626500 & C -3.16854200 & -1.00789300 & -0.06039700 \\
\hline H $\quad 3.86408100$ & -1.59260100 & -1.17606200 & C -3.05702500 & -2.25053100 & 0.58649600 \\
\hline C -0.86478000 & 2.47934300 & -0.01941600 & C -4.12072200 & -3.15351600 & 0.56764900 \\
\hline C -1.15404100 & 3.35337700 & -1.07136200 & C -5.30180700 & -2.83000400 & -0.10616100 \\
\hline C -0.51562500 & 2.98853700 & 1.23449700 & C -5.41695000 & -1.59975300 & -0.75756500 \\
\hline C -1.07672700 & 4.73937000 & -0.87262000 & C -4.35683000 & -0.68980000 & -0.73568500 \\
\hline H - 1.43908700 & 2.97298900 & -2.05052400 & H -4.10226800 & 0.82945500 & 1.73235400 \\
\hline C -0.44608900 & 4.37531800 & 1.43205500 & H -4.31017200 & 2.16985200 & 3.79604700 \\
\hline H -0.29316900 & 2.32363900 & 2.06626900 & H - 2.30122500 & 3.22817100 & 4.81000000 \\
\hline C -0.72322000 & 5.25155600 & 0.37895200 & H - 0.07294200 & 2.93195400 & 3.74220500 \\
\hline H -1.29700900 & 5.41560400 & -1.69638200 & H $\quad 0.14572900$ & 1.57644600 & 1.68255300 \\
\hline H - 0.17310900 & 4.76720800 & 2.41002300 & H -3.03990500 & 2.83221200 & -0.26621200 \\
\hline \multirow[t]{2}{*}{ H -0.66824000} & 6.32636100 & 0.53399000 & H -3.28975800 & 4.34335500 & -2.20112400 \\
\hline & & & H - 2.35303000 & 3.70950100 & -4.41645900 \\
\hline Int8 & & & H -1.15499200 & 1.54373100 & -4.68005100 \\
\hline C $\quad 3.40056400$ & 2.33460800 & 0.00355500 & H -0.88558300 & 0.03066500 & -2.74145700 \\
\hline O $\quad 4.36696700$ & 3.26983600 & -0.59300400 & H -2.13642800 & -2.51195400 & 1.10262300 \\
\hline C $\quad 5.70086500$ & 2.93574600 & -0.16992800 & H -4.02319400 & -4.11107100 & 1.07221800 \\
\hline C $\quad 5.53736300$ & 2.07116500 & 1.07767700 & H -6.12712800 & -3.53683300 & -0.12736400 \\
\hline C $\quad 4.26421300$ & 1.28734900 & 0.73559900 & H -6.33189200 & -1.34553400 & -1.28615400 \\
\hline H $\quad 2.81061500$ & 2.91124800 & 0.72776800 & H - 4.45872300 & 0.26288500 & -1.24653900 \\
\hline H $\quad 6.21910400$ & 2.38437700 & -0.96912100 & C $\quad 2.14651100$ & -2.07917400 & -0.08409400 \\
\hline H $\quad 6.24445500$ & 3.87077200 & 0.00831500 & C 2.84283200 & -2.31904300 & -1.28827700 \\
\hline H $\quad 6.40421500$ & 1.42921100 & 1.26405900 & C 2.72955300 & -2.59206700 & 1.09453600 \\
\hline H $\quad 5.37720300$ & 2.70076000 & 1.96130900 & C 4.05038400 & -3.02639300 & -1.31660500 \\
\hline H $\quad 4.49805400$ & 0.45655100 & 0.05899600 & H $\quad 2.44116700$ & -1.94699300 & -2.22906900 \\
\hline H $\quad 3.74946900$ & 0.87805400 & 1.60978200 & C $\quad 3.93639800$ & -3.30096600 & 1.07571000 \\
\hline C $\quad 2.51685100$ & 1.79192600 & -1.06025800 & H $\quad 2.23704000$ & -2.43742100 & 2.05296500 \\
\hline H $\quad 1.50023400$ & 2.14907500 & -1.19234200 & C $\quad 4.60346000$ & -3.52076800 & -0.13221800 \\
\hline H $\quad 2.94604100$ & 1.17076800 & -1.84264300 & H $\quad 4.55972500$ & -3.19104000 & -2.26433500 \\
\hline $\mathrm{Au} 0.37176000$ & -1.02949700 & -0.04481500 & H $\quad 4.35570100$ & -3.68135400 & 2.00533300 \\
\hline P - 1.73307700 & 0.13770800 & 0.01974700 & Н $\quad 5.54137300$ & -4.07115200 & -0.15090400 \\
\hline C -1.96385300 & 1.11722000 & 1.55736800 & & & \\
\hline C -3.21776100 & 1.28737500 & 2.16465600 & Int16 & & \\
\hline C -3.33545300 & 2.04635700 & 3.33145100 & C $\quad 1.38432500$ & -0.64083600 & -1.51488700 \\
\hline C -2.20668500 & 2.64140800 & 3.90013400 & O $\quad 2.78761500$ & -0.31886300 & -1.94851400 \\
\hline C -0.95496000 & 2.47535800 & 3.30112200 & C 3.21901900 & -1.22101700 & -3.00574000 \\
\hline C -0.83172600 & 1.71267600 & 2.13874600 & C 2.18308800 & -2.34121700 & -3.02601900 \\
\hline C -1.95557000 & 1.31789100 & -1.37046500 & C $\quad 0.90390200$ & -1.61059600 & -2.59669900 \\
\hline
\end{tabular}




\begin{tabular}{|c|c|c|c|c|c|}
\hline Н 1.49619200 & -1.16419000 & -0.55928100 & H -4.73648100 & -2.14107100 & 2.57128700 \\
\hline H $\quad 3.23464200$ & -0.65733900 & -3.94557500 & C -1.78124000 & 2.56371900 & -0.53971100 \\
\hline H $\quad 4.23509400$ & -1.55377800 & -2.77606900 & C -0.97598200 & 3.41863400 & 0.21771100 \\
\hline H 2.10413900 & -2.81200400 & -4.01061700 & C -2.83953500 & 3.08146700 & -1.29059300 \\
\hline H $\quad 2.44630800$ & -3.11473800 & -2.29490700 & C -1.24117100 & 4.79550600 & 0.23545800 \\
\hline H $\quad 0.47952300$ & -1.04820300 & -3.43838500 & H -0.14001400 & 3.03131300 & 0.79680300 \\
\hline H $\quad 0.13405400$ & -2.28720200 & -2.21442800 & C -3.09709200 & 4.45998500 & -1.27644400 \\
\hline C $\quad 0.61681300$ & 0.64673700 & -1.33980700 & H -3.47217800 & 2.42914200 & -1.88813500 \\
\hline Н 1.13404500 & 1.30233700 & -0.63036200 & C -2.30107500 & 5.31723200 & -0.51147400 \\
\hline H $\quad 0.53078500$ & 1.17730500 & -2.29633200 & H -0.61572800 & 5.45743600 & 0.83137900 \\
\hline $\mathrm{Au}-1.33768700$ & 0.32968900 & -0.55221800 & H -3.92150600 & 4.86021800 & -1.86349100 \\
\hline P -3.41453800 & -0.65935200 & 0.32947500 & H - 2.50350600 & 6.38547400 & -0.50040500 \\
\hline C -4.90598400 & -0.19230400 & -0.63597300 & $\mathrm{~N} \quad 4.49089300$ & -0.02360500 & -0.03754900 \\
\hline C -6.12348700 & 0.16280200 & -0.03778600 & H 3.73541000 & -0.19786800 & -0.84139100 \\
\hline C -7.21883600 & 0.51703400 & -0.83071900 & S $\quad 5.35918600$ & 1.40364200 & -0.15277100 \\
\hline C - 7.10984100 & 0.51633500 & -2.22262000 & O $\quad 6.65510900$ & 1.28612400 & 0.50424100 \\
\hline C -5.89873000 & 0.16294900 & -2.82592300 & O $\quad 5.22866800$ & 1.85030600 & -1.53544600 \\
\hline C -4.80043200 & -0.18274200 & -2.03825300 & C $\quad 4.33056200$ & 2.57877300 & 0.88582300 \\
\hline C -3.35678200 & -2.49865800 & 0.26442500 & F $\quad 4.15614100$ & 2.06455100 & 2.09917600 \\
\hline C -4.46174500 & -3.28001400 & -0.10626600 & F $\quad 3.15180400$ & 2.76843400 & 0.29914500 \\
\hline C -4.35925900 & -4.67356800 & -0.13611100 & F $\quad 4.98721100$ & 3.73201600 & 0.96741200 \\
\hline C -3.15863700 & -5.29827500 & 0.20794900 & S $\quad 4.69587000$ & -1.21141400 & 1.12106900 \\
\hline C -2.05423100 & -4.52615200 & 0.58061000 & O $\quad 5.33768600$ & -0.67285000 & 2.31376400 \\
\hline C -2.14966900 & -3.13435500 & 0.60254400 & O $\quad 3.43845400$ & -1.95015200 & 1.18658800 \\
\hline C -3.78654600 & -0.25677600 & 2.08414300 & C $\quad 5.93407300$ & -2.35764300 & 0.30474600 \\
\hline C -3.40294900 & 0.99949500 & 2.58180600 & F $\quad 7.00989900$ & -1.66697800 & -0.05787100 \\
\hline C -3.67469300 & 1.34536400 & 3.90683900 & F $\quad 5.37081300$ & -2.91788800 & -0.76330700 \\
\hline C -4.32280100 & 0.43874300 & 4.74952900 & F $\quad 6.26798900$ & -3.29502200 & 1.18655700 \\
\hline C -4.70230400 & -0.81477900 & 4.26275200 & & & \\
\hline C -4.43686700 & -1.16352100 & 2.93688700 & & & \\
\hline H -6.22363700 & 0.16408300 & 1.04308500 & {$\left[\mathrm{Ru}^{\mathrm{III}}\right]$} & & \\
\hline H -8.15704300 & 0.79272500 & -0.35645200 & $\mathrm{Ru} 0.01513500$ & -0.00023900 & 0.01153800 \\
\hline H -7.96339100 & 0.79240100 & -2.83609400 & $\mathrm{~N}-1.07040900$ & 1.52358400 & -0.99351000 \\
\hline H -5.80662700 & 0.16361500 & -3.90874000 & C -2.34272700 & 1.73661800 & -0.55756000 \\
\hline H -3.85769500 & -0.44514500 & -2.51303700 & C -3.14446400 & 2.70649200 & -1.16174400 \\
\hline H -5.40231200 & -2.80744500 & -0.37203700 & C -2.63194200 & 3.45815600 & -2.21644800 \\
\hline H -5.22073500 & -5.26880700 & -0.42735500 & C -1.32634500 & 3.23038400 & -2.64770700 \\
\hline H -3.08179100 & -6.38207100 & 0.18278400 & C -0.57344900 & 2.25329200 & -2.00754200 \\
\hline H - 1.11629000 & -5.00645500 & 0.84650000 & H -4.15705700 & 2.87590300 & -0.81682900 \\
\hline H - 1.28294000 & -2.53853500 & 0.87977900 & H -3.24837000 & 4.21396900 & -2.69248600 \\
\hline H - 2.89157600 & 1.70559700 & 1.93393500 & H -0.88866300 & 3.79383000 & -3.46413000 \\
\hline H -3.37294700 & 2.32013000 & 4.28090400 & H $\quad 0.44497000$ & 2.03855600 & -2.30730300 \\
\hline H -4.52799200 & 0.70601600 & 5.78281700 & N -0.77158000 & -1.69330900 & -0.97859600 \\
\hline H -5.20390600 & -1.52498600 & 4.91478700 & $\mathrm{~N} \quad 1.87189800$ & 0.15887700 & -0.98904500 \\
\hline
\end{tabular}




\begin{tabular}{|c|c|c|c|c|c|}
\hline $\mathrm{N}-1.84878300$ & 0.00271700 & 1.02138000 & H - 2.05961700 & -4.91923100 & -2.65487400 \\
\hline $\mathrm{N} \quad 0.94202400$ & 1.62077800 & 1.00378800 & H $\quad 5.31344200$ & 0.64915900 & -2.65981600 \\
\hline $\mathrm{N} \quad 0.95547100$ & -1.61456600 & 1.01302000 & H $\quad 2.79802800$ & 4.58943600 & 2.61723100 \\
\hline C -2.77427800 & 0.89313700 & 0.56926900 & H $\quad 2.49645600$ & -4.71101300 & 2.71907900 \\
\hline C -0.33965800 & -2.90539700 & -0.53341800 & & & \\
\hline C - 1.64588500 & -1.62234400 & -1.99803400 & {$\left[\mathrm{Ru}^{\mathrm{II}}\right]$} & & \\
\hline C $\quad 2.69575800$ & 1.15592900 & -0.56067900 & Ru-0.01156700 & 0.05921000 & -0.00817000 \\
\hline C $\quad 2.25876000$ & -0.65113100 & -1.99034300 & $\mathrm{~N}-1.76796000$ & -0.33839600 & -1.10732800 \\
\hline C -2.14239000 & -0.81302400 & 2.04954600 & C -2.51740200 & -1.38521100 & -0.66150800 \\
\hline C $\quad 2.17347100$ & 1.97590700 & 0.54530100 & C -3.70501800 & -1.74484600 & -1.30605200 \\
\hline C $\quad 0.37696400$ & 2.29799000 & 2.01911500 & C -4.12955800 & -1.02516700 & -2.41901300 \\
\hline C $\quad 0.61581900$ & -2.86158700 & 0.58471800 & C -3.35511400 & 0.04356100 & -2.86751300 \\
\hline C 1.83283800 & -1.46390900 & 2.02167500 & C - 2.18409100 & 0.35299900 & -2.18479800 \\
\hline C -4.03369500 & 0.96870400 & 1.16604800 & H -4.29347200 & -2.57846300 & -0.94333600 \\
\hline C -0.79674300 & -4.08265100 & -1.12741400 & H -5.05016000 & -1.29720300 & -2.92585300 \\
\hline C -2.13013800 & -2.76108500 & -2.63057500 & H -3.64413300 & 0.63320100 & -3.73098400 \\
\hline H -1.95043100 & -0.63183200 & -2.31277800 & H - 1.55183300 & 1.17366800 & -2.50256700 \\
\hline C $\quad 3.94622100$ & 1.34448500 & -1.15146600 & $\mathrm{~N} \quad 1.23808700$ & -1.28380800 & -1.04789300 \\
\hline C $\quad 3.48823900$ & -0.50370100 & -2.62107600 & $\mathrm{~N} \quad 0.55408700$ & 1.80077400 & -1.05597200 \\
\hline H $\quad 1.56325400$ & -1.42606100 & -2.28741600 & $\mathrm{~N}-0.82625500$ & -1.58830100 & 1.03211500 \\
\hline C -3.37696400 & -0.77566700 & 2.68662700 & $\mathrm{~N}-1.06305900$ & 1.56563300 & 1.03066100 \\
\hline H - 1.36637600 & -1.50077000 & 2.36264200 & $\mathrm{~N} \quad 1.78773500$ & 0.18531600 & 1.08643800 \\
\hline C $\quad 2.85682800$ & 3.05175000 & 1.11462000 & C -1.98753400 & -2.08807900 & 0.52343100 \\
\hline C 1.01647600 & 3.36907500 & 2.63125300 & C $\quad 2.49966600$ & -1.42045700 & -0.55126200 \\
\hline H - 0.60259900 & 1.96675100 & 2.34212000 & C $\quad 0.88088600$ & -1.99401400 & -2.13407800 \\
\hline C $\quad 1.16477500$ & -3.99312200 & 1.18995900 & C $\quad 0.01298100$ & 2.96456800 & -0.59769900 \\
\hline C $\quad 2.40731800$ & -2.55421000 & 2.66370300 & C 1.39894200 & 1.83929600 & -2.10298900 \\
\hline H $\quad 2.06819000$ & -0.44779800 & 2.31492200 & C -0.28742400 & -2.16627100 & 2.12198000 \\
\hline C -4.33629300 & 0.12855800 & 2.23469400 & C -0.90278900 & 2.83118500 & 0.55224800 \\
\hline H -4.77186300 & 1.67411200 & 0.80512000 & $\mathrm{C}-1.87057600$ & 1.36170100 & 2.08774300 \\
\hline C - 1.69960400 & -4.00950600 & -2.18519500 & C $\quad 2.80737600$ & -0.59799800 & 0.63554900 \\
\hline H - 0.45131900 & -5.04546700 & -0.77205100 & C 1.98691700 & 0.96360800 & 2.16634400 \\
\hline H - 2.82983000 & -2.65938900 & -3.45263200 & C -2.61524600 & -3.19007700 & 1.11261100 \\
\hline C $\quad 4.34410300$ & 0.50887400 & -2.19217200 & C $\quad 3.41868900$ & -2.28544500 & -1.15344500 \\
\hline H $\quad 4.60514400$ & 2.13188600 & -0.80649800 & C 1.74963900 & -2.86917100 & -2.77638400 \\
\hline H $\quad 3.75839400$ & -1.17525300 & -3.42826900 & $\mathrm{H}-0.13120700$ & -1.84682200 & -2.49196100 \\
\hline H -3.57173400 & -1.44639300 & 3.51596900 & C $\quad 0.32670300$ & 4.18807500 & -1.19730500 \\
\hline C $\quad 2.27340900$ & 3.75284200 & 2.16705600 & C 1.74861200 & 3.02422700 & -2.74101400 \\
\hline H $\quad 3.83212200$ & 3.34124500 & 0.74395000 & H $\quad 1.79937000$ & 0.88780500 & -2.43230500 \\
\hline H $\quad 0.52917100$ & 3.88594500 & 3.45038400 & C -0.86525100 & -3.26223200 & 2.75291500 \\
\hline C $\quad 2.06589900$ & -3.83743900 & 2.24024300 & H $\quad 0.63159500$ & -1.72868900 & 2.49350900 \\
\hline H $\quad 0.89199600$ & -4.98456700 & 0.85002200 & C -1.57052800 & 3.91085900 & 1.13875900 \\
\hline H 3.10573200 & -2.38919700 & 3.47644400 & C -2.55723000 & 2.39436700 & 2.71638500 \\
\hline H -5.31211400 & 0.18193300 & 2.70656200 & H - 1.95948600 & 0.33824900 & 2.43215800 \\
\hline
\end{tabular}




\begin{tabular}{|c|c|c|c|c|c|}
\hline C $\quad 4.04847600$ & -0.60231300 & 1.27997700 & C -2.79532500 & 0.49321900 & 0.66838000 \\
\hline C 3.19809100 & 1.00389900 & 2.84805300 & C -1.63427900 & -0.34848500 & 2.51245200 \\
\hline 1.14304700 & 1.56357100 & 2.48619700 & C 2.05116900 & -0.71515000 & -2.26281000 \\
\hline-2.04979900 & -3.78465400 & 2.23655800 & C -2.69585900 & 0.94021200 & -0.73341000 \\
\hline H -3.53665900 & -3.58281200 & 0.69966800 & $\mathrm{C}-1.28006300$ & 1.24982900 & -2.56429500 \\
\hline C $\quad 3.04191200$ & -3.01750800 & -2.27551100 & C $\quad 0.35946600$ & -2.90134300 & 0.27563200 \\
\hline H $\quad 4.42074900$ & -2.38670100 & -0.75478700 & C -1.23182900 & -2.44170300 & -1.41561200 \\
\hline H 1.41082600 & -3.41683400 & -3.64930800 & C $\quad 3.47562900$ & 1.61643900 & -2.11636100 \\
\hline C $\quad 1.20232500$ & 4.22038000 & -2.27856700 & C $\quad 2.07215100$ & -3.12511100 & 2.11831400 \\
\hline H - 0.10441000 & 5.10854900 & -0.82339300 & C $\quad 2.95873400$ & -1.09261800 & 3.07034400 \\
\hline H $\quad 2.43539300$ & 2.99888600 & -3.58039700 & H $\quad 2.14972300$ & 0.70292600 & 2.20673000 \\
\hline H -0.38825000 & -3.68922500 & 3.62861700 & C -3.98236900 & 0.52156900 & 1.40989500 \\
\hline C -2.40591900 & 3.69208000 & 2.23038600 & C -2.77998200 & -0.34152400 & 3.29890000 \\
\hline H - 1.44406800 & 4.91335500 & 0.74809100 & H - 0.68167300 & -0.68233500 & 2.90629200 \\
\hline H -3.19474300 & 2.17501300 & 3.56623500 & C 3.14627100 & -0.54628900 & -3.10178300 \\
\hline C $\quad 4.24767000$ & 0.20576700 & 2.39555300 & H $\quad 1.45701100$ & -1.62096400 & -2.28759100 \\
\hline H $\quad 4.85299600$ & -1.23088200 & 0.91780400 & C -3.77786400 & 1.44275300 & -1.46602500 \\
\hline H $\quad 3.30556700$ & 1.64860400 & 3.71374000 & C -2.31344900 & 1.76081300 & -3.34059600 \\
\hline H - 2.52990300 & -4.64070800 & 2.70022300 & H - 0.27684900 & 1.15025100 & -2.96271900 \\
\hline H 3.74889200 & -3.69121800 & -2.74946100 & C $\quad 0.16727500$ & -4.29548800 & 0.04624800 \\
\hline H $\quad 1.45224700$ & 5.16580200 & -2.74987400 & $\mathrm{C}-1.45170000$ & -3.78472200 & -1.66888600 \\
\hline H -2.92805300 & 4.52399400 & 2.69254500 & H - 1.76439900 & -1.67963100 & -1.97640700 \\
\hline H $\quad 5.20774100$ & 0.20855900 & 2.90199800 & C 3.87422300 & 0.64185100 & -3.02495900 \\
\hline & & & H $\quad 4.02668300$ & 2.54643600 & -2.04490600 \\
\hline$\left[\mathrm{Ru}^{\mathrm{I}}\right]$ & & & C $\quad 2.90906700$ & -2.51023400 & 3.01990500 \\
\hline Ru 0.04529600 & 0.03103800 & -0.04273700 & H $\quad 2.02624300$ & -4.20818500 & 2.07428600 \\
\hline $\mathrm{N} \quad 0.74977200$ & 1.98105800 & 0.37742800 & H $\quad 3.60526700$ & -0.56675200 & 3.76473900 \\
\hline C $\quad 1.85206200$ & 2.37075600 & -0.32466900 & C -3.97790600 & 0.09947100 & 2.73514500 \\
\hline C $\quad 2.42891300$ & 3.62949400 & -0.11892900 & H -4.90352700 & 0.87015600 & 0.95872000 \\
\hline C $\quad 1.86871600$ & 4.50119300 & 0.80895100 & H -2.72637000 & -0.67888500 & 4.32862600 \\
\hline C $\quad 0.73503200$ & 4.09741800 & 1.51522800 & H $\quad 3.41782800$ & -1.33572400 & -3.79463500 \\
\hline C $\quad 0.21088000$ & 2.83385000 & 1.26978000 & C -3.58782100 & 1.85651500 & -2.78034500 \\
\hline H $\quad 3.30748000$ & 3.92824700 & -0.67808300 & H -4.76071300 & 1.51287800 & -1.01599500 \\
\hline H $\quad 2.30999900$ & 5.47909200 & 0.97514400 & H -2.11559100 & 2.07100100 & -4.36123300 \\
\hline H $\quad 0.26078700$ & 4.74270500 & 2.24718700 & C -0.72084800 & -4.73407300 & -0.90793500 \\
\hline H -0.66371900 & 2.47725400 & 1.80140000 & H $\quad 0.73106400$ & -5.01457800 & 0.63127200 \\
\hline $\mathrm{N} \quad 1.31850300$ & -0.96040500 & 1.30851900 & H -2.16331000 & -4.08621000 & -2.43005100 \\
\hline $\mathrm{N}-1.63112600$ & 0.05314400 & 1.22668800 & H $\quad 4.73724600$ & 0.80949200 & -3.66188400 \\
\hline $\mathrm{N} \quad 1.66110800$ & 0.22147300 & -1.37673800 & H $\quad 3.52567700$ & -3.10707900 & 3.68687200 \\
\hline $\mathrm{N}-1.45532700$ & 0.84418100 & -1.29206100 & H -4.89457500 & 0.11716900 & 3.31650500 \\
\hline $\mathrm{N}-0.36455800$ & -1.98486600 & -0.48936800 & H -4.42149900 & 2.24729600 & -3.35550700 \\
\hline C $\quad 2.36226100$ & 1.38898500 & -1.29885400 & H - 0.85965500 & -5.79881100 & -1.07617700 \\
\hline C $\quad 1.25786300$ & -2.35367700 & 1.23803900 & & & \\
\hline C $\quad 2.14950400$ & -0.38284700 & 2.19995400 & $*\left[\mathrm{Ru}^{\mathrm{II}}\right]$ & & \\
\hline
\end{tabular}




\begin{tabular}{|c|c|c|c|c|c|c|c|}
\hline \multicolumn{2}{|c|}{$\mathrm{Ru}-0.00126900$} & \multirow{2}{*}{$\begin{array}{r}-0.07134200 \\
1.54854400\end{array}$} & \multirow{2}{*}{$\begin{array}{l}0.00224700 \\
-0.97957000\end{array}$} & \multirow{2}{*}{$\begin{array}{l}\mathrm{H} \\
\mathrm{H}\end{array}$} & \multirow{2}{*}{$\begin{array}{l}-0.69355600 \\
-3.20008100\end{array}$} & \multirow{2}{*}{$\begin{array}{l}-5.10123100 \\
-2.62267500\end{array}$} & \multirow{2}{*}{$\begin{array}{l}-0.80200100 \\
-3.26894000\end{array}$} \\
\hline $\mathrm{N}$ & -1.10644100 & & & & & & \\
\hline $\mathrm{C}$ & -2.37717100 & 1.89438400 & -0.64458000 & $\mathrm{C}$ & 4.96114300 & 0.37215500 & -1.70774400 \\
\hline 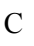 & -3.01476700 & 2.96574500 & -1.28300500 & $\mathrm{H}$ & 5.06939400 & 1.80709300 & -0.11357100 \\
\hline $\mathrm{C}$ & -2.35385200 & 3.67249300 & -2.28237900 & $\mathrm{H}$ & 4.50354000 & -1.10622300 & -3.22890500 \\
\hline $\mathrm{C}$ & -1.05597500 & 3.29995300 & -2.62646400 & $\mathrm{H}$ & -4.51775700 & -1.06687900 & 3.22727200 \\
\hline 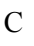 & -0.47090700 & 2.23941600 & -1.94537900 & $\mathrm{C}$ & 2.38705900 & 3.64733100 & 2.28620600 \\
\hline$\Pi$ & -4.01816800 & 3.25418800 & -0.99488700 & $\mathrm{H}$ & 4.04681000 & 3.21256200 & 0.99810800 \\
\hline 1 & -2.84578900 & 4.50303400 & -2.77926200 & $\mathrm{H}$ & 0.53270100 & 3.81096000 & 3.40321300 \\
\hline $\mathrm{H}$ & -0.49803900 & 3.81725800 & -3.39961900 & $\mathrm{C}$ & 1.98260000 & -4.04137300 & 2.09653300 \\
\hline $\mathrm{H}$ & 0.54024100 & 1.91919000 & -2.170 & $\mathrm{H}$ & 0.65059800 & -5.10871500 & 0.79144500 \\
\hline $\mathrm{N}$ & -0.95772900 & -1.73328000 & -0.91842500 & $\mathrm{H}$ & 3.17938700 & -2.65778400 & 3.26341000 \\
\hline $1 \mathrm{v}$ & 2.25271600 & 0.23101200 & -1.10681500 & $\mathrm{H}$ & -6.01800500 & 0.49617300 & 1.94456100 \\
\hline $\mathrm{N}$ & -2.25543900 & 0.24669300 & 1.10266700 & $\mathrm{H}$ & -2.42331400 & -4.91347600 & -2.56585400 \\
\hline $\mathrm{N}$ & 1.11823000 & 1.53620700 & 0.98318900 & $\mathrm{H}$ & 6.02224400 & 0.42703200 & -1.93213500 \\
\hline $1 \mathrm{~s}$ & 0.94421600 & -1.74347500 & 0.91560000 & $\mathrm{H}$ & 2.88732300 & 4.47287700 & 2.78309400 \\
\hline $\mathrm{C}$ & -3.04408500 & 1.09757300 & 0.41691300 & $\mathrm{H}$ & 2.38224000 & -4.94015900 & 2.55564300 \\
\hline$C$ & -0.52708900 & -2.96590900 & -0.52 & & & & \\
\hline $\mathrm{C}$ & -1.89601300 & -1.63770000 & -1.87996500 & Ints & & & \\
\hline$C$ & 3.05146700 & 1.06657400 & -0.41385600 & $C-1$ & 3.78105600 & -0.82296900 & -0.58875000 \\
\hline $\mathrm{C}$ & 2.77528500 & -0.52214600 & -2.08248600 & $\mathrm{O}-$ & 5.19597200 & -0.59268700 & -0.84301700 \\
\hline $\mathrm{C}$ & -2.78540200 & -0.50330700 & 2.07672300 & $\mathrm{C}-\mathrm{S}$ & 5.97900800 & -1.18061400 & 0.21193700 \\
\hline $\mathrm{C}$ & 2.39242700 & 1.86929700 & 0.64815100 & $\mathrm{C}-\mathrm{s}$ & 1365100 & -2.30316000 & 0.77564200 \\
\hline $\mathrm{C}$ & 0.48960800 & 2.23352600 & 1.94895600 & $\mathrm{C}-\mathrm{T}$ & 3.71513000 & -1.67284400 & 0.69582600 \\
\hline$C$ & 0.50289400 & -2.97151100 & 0.52240100 & $\Pi$ & 3.39659500 & -1.38300800 & -1.45053100 \\
\hline $\mathrm{C}$ & 1.88366300 & -1.65833300 & 1.87701200 & $\mathrm{H}-($ & 6.20638600 & -0.42715500 & 0.98083900 \\
\hline $\mathrm{C}$ & -4.41363300 & 1.20452600 & 0.69737200 & $\mathrm{H}-\mathrm{C}$ & 6.92238200 & -1.52137000 & -0.22741600 \\
\hline $\mathrm{C}$ & -1.04662200 & -4.12525900 & -1.11222700 & - & 5.39966800 & -2.59046600 & 1.79219700 \\
\hline $\mathrm{C}$ & -2.44882700 & -2.75286100 & -2.49744100 & $\mathrm{H}-$ & 5.17771800 & -3.18965800 & 0.13373800 \\
\hline $\mathrm{H}$ & -2.19985400 & -0.63521700 & -2.15631500 & $\mathrm{H}-$ & 3.54162000 & -1.02611700 & 1.56477900 \\
\hline $\mathrm{C}$ & 4.42367700 & 1.15406600 & -0.68816300 & $\mathrm{H}-$ & 2.90983700 & -2.41349400 & 0.65891100 \\
\hline $\mathrm{C}$ & 4.12464700 & -0.48162800 & -2.42647600 & $\mathrm{C}-\mathrm{T}$ & 3.10296400 & 0.54124300 & -0.50091300 \\
\hline $\mathrm{H}$ & 2.08381200 & -1.17938400 & -2.60380800 & $\mathrm{H}-$ & 3.21345900 & 1.11109700 & -1.42728800 \\
\hline $\mathrm{C}$ & -4.13261100 & -0.44410700 & 2.42641500 & $\mathrm{H}-$ & 3.48265200 & 1.12265600 & 0.34277000 \\
\hline $\mathrm{H}$ & -2.10183700 & -1.17353300 & 2.59188900 & $\mathrm{Au}-$ & -1.02906800 & 0.21151800 & -0.24944000 \\
\hline $\mathrm{C}$ & 3.04076900 & 2.93406700 & 1.28671500 & $\mathrm{P}$ & 1.39254800 & -0.47932500 & 0.00062300 \\
\hline$C$ & 1.08540500 & 3.28800800 & 2.63010000 & $c$ & 2.33599200 & 0.44482800 & 1.26835500 \\
\hline $\mathrm{H}$ & -0.52473100 & 1.92359700 & 2.17384000 & $\mathrm{C}$ & 3.55254800 & 1.08015400 & 0.98042600 \\
\hline $\mathrm{C}$ & 1.01237800 & -4.13665900 & 1.10390500 & $\mathrm{C}$ & 4.22308800 & 1.79345600 & 1.97784800 \\
\hline $\mathrm{C}$ & 2.42681100 & -2.77970700 & 2.49183300 & $\mathrm{C}$ & 3.68887300 & 1.87504100 & 3.26474800 \\
\hline $\mathrm{H}$ & 2.19653800 & -0.65936000 & 2.15589300 & $\mathrm{C}$ & 2.47502900 & 1.24473500 & 3.55678800 \\
\hline $\mathrm{C}$ & -4.95880500 & 0.42603400 & 1.71545300 & $\mathrm{C}$ & 1.79634100 & 0.53932900 & 2.56377000 \\
\hline $\mathrm{H}$ & -5.05177100 & 1.87001300 & 0.12869200 & $\mathrm{C}$ & 1.41868700 & -2.24185600 & 0.50293400 \\
\hline $\mathrm{C}$ & -2.01581100 & -4.01924400 & -2.10476300 & $\mathrm{C}$ & 2.36090900 & -2.76808800 & 1.39942000 \\
\hline
\end{tabular}




\begin{tabular}{|c|c|c|c|c|c|c|}
\hline $\mathrm{C}$ & 2.32503900 & -4.12406200 & 1.73372400 & H $\quad 5.22373100$ & 2.97448300 & -0.54771300 \\
\hline $\mathrm{C}$ & 1.35394700 & -4.96124100 & 1.17899800 & H $\quad 4.49860400$ & 4.43886900 & -1.25681600 \\
\hline $\mathrm{C}$ & 0.41199800 & -4.44243100 & 0.28593500 & H 4.26355100 & 4.02051100 & 1.44997900 \\
\hline $\mathrm{C}$ & 0.44085400 & -3.08807700 & -0.04816400 & H $\quad 2.84474200$ & 4.58094500 & 0.53694800 \\
\hline $\mathrm{C}$ & 2.33207500 & -0.37247100 & -1.56754700 & H $\quad 3.55751800$ & 1.69594500 & 1.28744400 \\
\hline $\mathrm{C}$ & 1.95832900 & 0.59711700 & -2.51229000 & H $\quad 1.98718000$ & 2.49086600 & 1.50167400 \\
\hline C & 2.65668600 & 0.71051700 & -3.71592900 & C 2.75112900 & 0.54450100 & -0.93879200 \\
\hline $\mathrm{C}$ & 3.72568900 & -0.14652400 & -3.98778000 & H $\quad 2.45823000$ & 0.25952300 & -1.95011000 \\
\hline$C$ & 4.09939900 & -1.11629200 & -3.05290100 & H $\quad 3.78828100$ & 0.28850200 & -0.75170900 \\
\hline $\mathrm{C}$ & 3.40778500 & -1.23184500 & -1.84606100 & $\mathrm{Au} 0.90529700$ & -0.46545600 & -0.01544800 \\
\hline 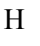 & 3.98191900 & 1.02117200 & -0.01460300 & P - 1.46640400 & 0.03374200 & -0.07457900 \\
\hline $\mathrm{H}$ & 5.16496600 & 2.28274100 & 1.74483900 & C - 2.20297900 & 0.04861300 & 1.60216300 \\
\hline $\mathrm{H}$ & 4.21356700 & 2.42906600 & 4.03844200 & C -3.55743100 & -0.25279000 & 1.81941800 \\
\hline $\mathrm{H}$ & 2.05408700 & 1.30626600 & 4.55662600 & C - 4.07929400 & -0.22803900 & 3.11394100 \\
\hline $\mathrm{H}$ & 0.85232700 & 0.05445400 & 2.80033600 & C -3.25764100 & 0.09457500 & 4.19745200 \\
\hline $\mathrm{H}$ & 3.11917900 & -2.12681200 & 1.83797900 & C -1.90914100 & 0.39320000 & 3.98728400 \\
\hline $\mathrm{H}$ & 3.05808800 & -4.52473300 & 2.42866500 & C -1.38079200 & 0.36650600 & 2.69555800 \\
\hline $\mathrm{H}$ & 1.32913000 & -6.01488800 & 1.44351700 & C -1.84928000 & 1.64426500 & -0.86152700 \\
\hline & -0.34656000 & -5.08914300 & -0.14652400 & C -2.75257200 & 2.55882300 & -0.30180200 \\
\hline & -0.29438900 & -2.68907300 & -0.74478300 & C -3.00732400 & 3.77247900 & -0.94642100 \\
\hline $\mathrm{H}$ & 1.12477100 & 1.26293100 & -2.30744100 & C -2.36724400 & 4.07872400 & -2.14869100 \\
\hline $\mathrm{H}$ & 2.36104300 & 1.46357100 & -4.44128600 & C - 1.46466400 & 3.16986700 & -2.71004600 \\
\hline $\mathrm{H}$ & 4.26501800 & -0.06180400 & -4.92740900 & C -1.20036700 & 1.96027600 & -2.06809100 \\
\hline $\mathrm{H}$ & 4.92923400 & -1.78562400 & -3.26301800 & C -2.37030700 & -1.24023100 & -1.03473200 \\
\hline $\mathrm{H}$ & 3.70537700 & -1.98981100 & -1.12751200 & C - 1.91211800 & -2.56706500 & -0.97537500 \\
\hline & -0.97849400 & 2.24029700 & -0.01342500 & C -2.58422900 & -3.57329200 & -1.66987100 \\
\hline & -0.97197700 & 3.05697500 & -1.14044800 & C -3.71109800 & -3.26213600 & -2.43616000 \\
\hline & -0.87893700 & 2.74360900 & 1.28026200 & -4.16711500 & -1.94349200 & -2.50356700 \\
\hline & -0.82029600 & 4.43751600 & -0.95547500 & C -3.50158500 & -0.93273800 & -1.80583600 \\
\hline & -1.07588300 & 2.64897600 & -2.13995700 & H -4.20365100 & -0.50921200 & 0.98534000 \\
\hline & -0.73277300 & 4.12789900 & 1.44132400 & H -5.12735900 & -0.46492700 & 3.27535400 \\
\hline & -0.90563700 & 2.09255600 & 2.14685800 & H -3.66660200 & 0.10810800 & 5.20410000 \\
\hline & -0.70082200 & 4.97152300 & 0.32919300 & H - 1.26597400 & 0.63914800 & 4.82778700 \\
\hline & -0.80238300 & 5.08841300 & -1.82569400 & H -0.32855900 & 0.58805300 & 2.53595200 \\
\hline & -0.64703500 & 4.53633100 & 2.44484000 & H -3.25937400 & 2.33094000 & 0.63059700 \\
\hline \multirow{2}{*}{\multicolumn{2}{|c|}{ H -0.58955000 }} & 6.04382800 & 0.46399000 & H -3.70814200 & 4.47615000 & -0.50550200 \\
\hline & & & & H -2.56714600 & 5.02365300 & -2.64671100 \\
\hline \multicolumn{4}{|c|}{$\mathrm{TS} 2$} & H -0.96119200 & 3.40510600 & -3.64375600 \\
\hline $\mathrm{C}$ & 2.48354200 & 2.00199900 & -0.58125700 & H -0.49216100 & 1.26091900 & -2.50577500 \\
\hline $\mathrm{O}$ & 3.35795300 & 2.74473300 & -1.46846200 & H - 1.02995400 & -2.81186000 & -0.38796900 \\
\hline $\mathrm{C}$ & 4.28489000 & 3.52867200 & -0.68776200 & H -2.22343600 & -4.59693500 & -1.61858400 \\
\hline $\mathrm{C}$ & 3.57350900 & 3.76842400 & 0.63908900 & H -4.22960400 & -4.04547300 & -2.98246000 \\
\hline $\mathrm{C}$ & 2.86329200 & 2.42220100 & 0.85072400 & H -5.04122800 & -1.69770400 & -3.10047400 \\
\hline $\mathrm{H}$ & 1.45752100 & 2.28820900 & -0.82939400 & H -3.86422000 & 0.08895900 & -1.86495700 \\
\hline
\end{tabular}




\begin{tabular}{|c|c|c|c|c|c|c|c|}
\hline $\mathrm{C}$ & 2.74490800 & -1.39264400 & 0.28068300 & $\mathrm{H}$ & -0.50901600 & 5.76464600 & 0.87194000 \\
\hline $\mathrm{C}$ & 3.08584900 & -2.43878100 & -0.58617100 & $\mathrm{H}$ & 1.28649400 & 5.37282600 & 2.54783200 \\
\hline $\mathrm{C}$ & 3.31140500 & -1.30235800 & 1.55861100 & $\mathrm{H}$ & 1.80857000 & 3.05420900 & 3.28564300 \\
\hline $\mathrm{C}$ & 3.95740900 & -3.43683700 & -0.14150700 & $\mathrm{H}$ & 0.56055700 & 1.14276300 & 2.34551500 \\
\hline $\mathrm{H}$ & 2.67975500 & -2.47924000 & -1.59260200 & $\mathrm{H}$ & -4.37470200 & 1.93394800 & -0.10822500 \\
\hline $\mathrm{C}$ & 4.18110500 & -2.30822100 & 1.99058100 & $\mathrm{H}$ & -5.47500700 & 2.73741900 & -2.16575700 \\
\hline $\mathrm{H}$ & 3.07610700 & -0.46882700 & 2.21231900 & $\mathrm{H}$ & -4.23236600 & 2.77493600 & -4.31909500 \\
\hline $\mathrm{C}$ & 4.50444000 & -3.37230400 & 1.14362100 & $\mathrm{H}$ & -1.86838200 & 2.00214300 & -4.40092400 \\
\hline $\mathrm{H}$ & 4.21226200 & -4.26008100 & -0.80389800 & $\mathrm{H}$ & -0.75992500 & 1.18155000 & -2.34945200 \\
\hline $\mathrm{H}$ & 4.61073900 & -2.25295000 & 2.98748400 & $\mathrm{H}$ & -2.08424200 & 1.66024600 & 3.25045000 \\
\hline \multirow[t]{2}{*}{$\mathrm{H}$} & 5.19106700 & -4.14428500 & 1.48033400 & $\mathrm{H}$ & -3.74096300 & 0.88604900 & 4.89920300 \\
\hline & & & & $\mathrm{H}$ & -5.46694600 & -0.78716000 & 4.25571100 \\
\hline \multicolumn{2}{|c|}{ Int 10} & & & $\mathrm{H}$ & -5.51836000 & -1.67808500 & 1.93134900 \\
\hline \multicolumn{2}{|c|}{$\mathrm{Au}-0.04832700$} & -1.01577100 & -0.31320800 & $\mathrm{H}$ & -3.86052500 & -0.92282400 & 0.27486700 \\
\hline $\mathrm{P}$ & -1.60547500 & 0.93380800 & 0.40662300 & $\mathrm{H}$ & -1.29369100 & -3.47596600 & 1.06187500 \\
\hline $\mathrm{C}$ & -1.55633300 & -2.32714300 & -0.75826100 & $\mathrm{~N}$ & 2.12667700 & -0.24133700 & -0.09647700 \\
\hline $\mathrm{C}$ & -1.82669500 & -3.38939500 & 0.11847000 & $\mathrm{~S}$ & 2.99690700 & -0.75082400 & 1.21160600 \\
\hline $\mathrm{C}$ & -2.78213400 & -4.35536000 & -0.21812500 & $\mathrm{O}$ & 4.27882000 & -0.06202600 & 1.38126700 \\
\hline $\mathrm{C}$ & -3.48647000 & -4.25997500 & -1.42121200 & $\mathrm{O}$ & 2.06434200 & -0.84803500 & 2.33990400 \\
\hline $\mathrm{C}$ & -3.23100600 & -3.19563600 & -2.28959400 & $\mathrm{C}$ & 3.41831300 & -2.53275200 & 0.80069100 \\
\hline $\mathrm{C}$ & -2.27570900 & -2.22724000 & -1.95784600 & $\mathrm{~F}$ & 3.99999800 & -2.61215000 & -0.39457500 \\
\hline $\mathrm{C}$ & -0.70204900 & 2.36966700 & 1.08351800 & $\mathrm{~F}$ & 2.30285100 & -3.26635800 & 0.80064400 \\
\hline $\mathrm{C}$ & -0.99168500 & 3.67947100 & 0.66556100 & $\mathrm{~F}$ & 4.25079800 & -2.99014400 & 1.73583900 \\
\hline $\mathrm{C}$ & -0.27683100 & 4.75410200 & 1.19698500 & $\mathrm{~S}$ & 2.85971800 & 0.41917400 & -1.42169200 \\
\hline $\mathrm{C}$ & 0.72978900 & 4.53332700 & 2.14020100 & $\mathrm{O}$ & 4.27857500 & 0.07753300 & -1.55861600 \\
\hline $\mathrm{C}$ & 1.02352700 & 3.23161700 & 2.55580900 & $\mathrm{O}$ & 1.95418600 & 0.22778300 & -2.56054100 \\
\hline $\mathrm{C}$ & 0.31613300 & 2.15135400 & 2.02769200 & $\mathrm{C}$ & 2.81698700 & 2.25914900 & -1.06887500 \\
\hline $\mathrm{C}$ & -2.48865200 & 1.50352800 & -1.08551200 & $\mathrm{~F}$ & 3.29502100 & 2.50576500 & 0.14958300 \\
\hline $\mathrm{C}$ & -3.82185000 & 1.94329700 & -1.04183900 & $\mathrm{~F}$ & 1.56381700 & 2.70315500 & -1.15859600 \\
\hline $\mathrm{C}$ & -4.44326300 & 2.39915300 & -2.20625500 & $\mathrm{~F}$ & 3.57251200 & 2.87383900 & -1.97955500 \\
\hline $\mathrm{C}$ & -3.74360000 & 2.42175500 & -3.41511400 & & & & \\
\hline $\mathrm{C}$ & -2.41566700 & 1.98790600 & -3.46248100 & Int & & & \\
\hline $\mathrm{C}$ & -1.78928500 & 1.52574600 & -2.30478200 & & -0.04929600 & -0.70563000 & -0.30912200 \\
\hline $\mathrm{C}$ & -2.85039400 & 0.42575000 & 1.64188700 & $\mathrm{P}$ & -1.78123300 & 0.78855800 & 0.41189600 \\
\hline $\mathrm{C}$ & -2.82610900 & 0.92642000 & 2.95442000 & $\mathrm{C}$ & -1.54461700 & -1.96595200 & -0.87781300 \\
\hline $\mathrm{C}$ & -3.76670400 & 0.48830000 & 3.88842900 & $\mathrm{C}$ & -1.82482300 & -3.02458000 & -0.01524100 \\
\hline $\mathrm{C}$ & -4.73629000 & -0.44880000 & 3.52609500 & $\mathrm{C}$ & -2.60902500 & -4.07684300 & -0.50159100 \\
\hline $\mathrm{C}$ & -4.76507500 & -0.95083200 & 2.22133900 & $\mathrm{C}$ & -3.11976000 & -4.03345600 & -1.80169000 \\
\hline $\mathrm{C}$ & -3.82566500 & -0.52459100 & 1.28373300 & $\mathrm{C}$ & -2.84854300 & -2.94237700 & -2.63016200 \\
\hline $\mathrm{H}$ & -2.97653900 & -5.17905700 & 0.46504400 & $\mathrm{C}$ & -2.06188000 & -1.87940500 & -2.16624100 \\
\hline $\mathrm{H}$ & -4.23282300 & -5.00748300 & -1.67815000 & $\mathrm{C}$ & -0.78491900 & 2.05874300 & 1.23210000 \\
\hline $\mathrm{H}$ & -3.77640400 & -3.11263900 & -3.22697200 & $\mathrm{C}$ & -0.99937800 & 3.41290400 & 0.91722500 \\
\hline $\mathrm{H}$ & -2.09961500 & -1.39985100 & -2.63956100 & $\mathrm{C}$ & -0.26448100 & 4.39440900 & 1.58116600 \\
\hline $\mathrm{H}$ & -1.77090100 & 3.86496100 & -0.06628300 & $\mathrm{C}$ & 0.68622200 & 4.03528000 & 2.53964100 \\
\hline
\end{tabular}




\begin{tabular}{|c|c|c|c|c|c|c|c|}
\hline $\mathrm{C}$ & 0.90436200 & 2.68818700 & 2.84582700 & $\mathrm{O}$ & 1.96297800 & 0.59794900 & -2.45795100 \\
\hline $\mathrm{C}$ & 0.17368700 & 1.69600000 & 2.19583600 & $\mathrm{C}$ & 2.97075300 & 2.33947800 & -0.70753800 \\
\hline $\mathrm{C}$ & -2.57649900 & 1.48221000 & -1.05118000 & $\mathrm{~F}$ & 3.49356100 & 2.37490500 & 0.51588300 \\
\hline $\mathrm{C}$ & -3.93752800 & 1.83141600 & -1.04054100 & $\mathrm{~F}$ & 1.73896100 & 2.84587500 & -0.68502000 \\
\hline $\mathrm{C}$ & -4.49636000 & 2.43622300 & -2.16690400 & $\mathrm{~F}$ & 3.73011700 & 3.04525800 & -1.54346000 \\
\hline $\mathrm{C}$ & -3.71080800 & 2.69045000 & -3.29329600 & & & & \\
\hline $\mathrm{C}$ & -2.35462500 & 2.34785400 & -3.30015100 & \multicolumn{2}{|c|}{ Int12 } & & \\
\hline $\mathrm{C}$ & -1.78348700 & 1.74324200 & -2.18334500 & $\mathrm{Au}$ & 0.02498500 & -0.02568800 & -0.06961100 \\
\hline $\mathrm{C}$ & -2.98047500 & 0.07240000 & 1.55840600 & $\mathrm{P}$ & -2.35676900 & -0.26396000 & -0.21701900 \\
\hline $\mathrm{C}$ & -2.88988500 & 0.35216700 & 2.93428200 & $\mathrm{C}$ & -3.09812100 & -0.01426300 & 1.42297000 \\
\hline $\mathrm{C}$ & -3.82710400 & -0.19021100 & 3.81253300 & $\mathrm{C}$ & -4.49156200 & -0.14919600 & 1.57093800 \\
\hline $\mathrm{C}$ & -4.85234600 & -1.00596500 & 3.33033700 & $\mathrm{C}$ & -5.07547700 & 0.04004600 & 2.82261300 \\
\hline $\mathrm{C}$ & -4.94627400 & -1.28261500 & 1.96227500 & $\mathrm{C}$ & -4.28011800 & 0.34418900 & 3.93126600 \\
\hline $\mathrm{C}$ & -4.01237900 & -0.75623800 & 1.07450100 & $\mathrm{C}$ & -2.89592600 & 0.45868500 & 3.79029800 \\
\hline $\mathrm{H}$ & -2.82315100 & -4.92219000 & 0.14677100 & $\mathrm{C}$ & -2.30178900 & 0.28066300 & 2.53964500 \\
\hline $\mathrm{H}$ & -3.74005100 & -4.84781300 & -2.16568800 & $\mathrm{C}$ & -2.87144900 & 1.03242900 & -1.39196100 \\
\hline $\mathrm{H}$ & -3.24935000 & -2.90398200 & -3.63939000 & $\mathrm{C}$ & -3.90130700 & 1.93406800 & -1.08280800 \\
\hline $\mathrm{H}$ & -1.86171300 & -1.02559800 & -2.80490800 & $\mathrm{C}$ & -4.28320500 & 2.89555900 & -2.02093700 \\
\hline $\mathrm{H}$ & -1.73298300 & 3.70172500 & 0.17269500 & $\mathrm{C}$ & -3.64263000 & 2.96688400 & -3.25917200 \\
\hline $\mathrm{H}$ & -0.43493400 & 5.44040800 & 1.34383800 & $\mathrm{C}$ & -2.60740000 & 2.07791400 & -3.56399000 \\
\hline $\mathrm{H}$ & 1.26082000 & 4.80539100 & 3.04651800 & $\mathrm{C}$ & -2.21427900 & 1.11721200 & -2.63336800 \\
\hline $\mathrm{H}$ & 1.64644400 & 2.40791500 & 3.58738300 & $\mathrm{C}$ & -2.98135800 & -1.86305000 & -0.83249700 \\
\hline $\mathrm{H}$ & 0.35499700 & 0.65198800 & 2.42906000 & $\mathrm{C}$ & -3.19837000 & -2.90646100 & 0.08589800 \\
\hline $\mathrm{H}$ & -4.55370400 & 1.64710200 & -0.16763900 & $\mathrm{C}$ & -3.67628300 & -4.13667800 & -0.36026800 \\
\hline $\mathrm{H}$ & -5.54781100 & 2.70782100 & -2.16022100 & $\mathrm{C}$ & -3.93819800 & -4.33916700 & -1.71852600 \\
\hline $\mathrm{H}$ & -4.15501800 & 3.15705300 & -4.16798400 & $\mathrm{C}$ & -3.72549900 & -3.30474500 & -2.63167400 \\
\hline $\mathrm{H}$ & -1.74408700 & 2.54616900 & -4.17603600 & $\mathrm{C}$ & -3.24852200 & -2.06781900 & -2.19589300 \\
\hline $\mathrm{H}$ & -0.73238300 & 1.46907800 & -2.18524000 & $\mathrm{H}$ & -5.11666000 & -0.40177500 & 0.72011300 \\
\hline $\mathrm{H}$ & -2.10725200 & 0.99388900 & 3.32139200 & $\mathrm{H}$ & -6.15176300 & -0.05737800 & 2.93117900 \\
\hline $\mathrm{H}$ & -3.75523800 & 0.03179900 & 4.87305900 & $\mathrm{H}$ & -4.74024600 & 0.48454400 & 4.90540800 \\
\hline $\mathrm{H}$ & -5.58019700 & -1.42605100 & 4.01876300 & $\mathrm{H}$ & -2.27420100 & 0.68360900 & 4.65202100 \\
\hline $\mathrm{H}$ & -5.74545800 & -1.91374500 & 1.58535100 & $\mathrm{H}$ & -1.22504600 & 0.36533400 & 2.43749300 \\
\hline $\mathrm{H}$ & -4.09481300 & -0.98152900 & 0.01700400 & $\mathrm{H}$ & -4.40645000 & 1.89536000 & -0.12420200 \\
\hline $\mathrm{H}$ & -1.44278500 & -3.04696100 & 1.00049700 & $\mathrm{H}$ & -5.08256100 & 3.58944200 & -1.77740000 \\
\hline $\mathrm{N}$ & 2.14929500 & -0.24226200 & -0.10054400 & $\mathrm{H}$ & -3.94358600 & 3.71777200 & -3.98423900 \\
\hline $\mathrm{S}$ & 3.00592900 & -0.96046200 & 1.13004900 & $\mathrm{H}$ & -2.09905800 & 2.13570500 & -4.52207400 \\
\hline $\mathrm{O}$ & 4.31818800 & -0.35735600 & 1.36098700 & $\mathrm{H}$ & -1.39645300 & 0.44201100 & -2.87010500 \\
\hline $\mathrm{O}$ & 2.07174600 & -1.15312400 & 2.24282400 & $\mathrm{H}$ & -2.99837800 & -2.76401800 & 1.14220500 \\
\hline $\mathrm{C}$ & 3.31720000 & -2.68541000 & 0.46268800 & $\mathrm{H}$ & -3.84554100 & -4.93611500 & 0.35525100 \\
\hline $\mathrm{F}$ & 4.00099000 & -2.62284600 & -0.67627300 & $\mathrm{H}$ & -4.31068600 & -5.30005900 & -2.06250600 \\
\hline F & 2.14485900 & -3.28827100 & 0.24856500 & $\mathrm{H}$ & -3.93614400 & -3.45364000 & -3.68680700 \\
\hline $\mathrm{F}$ & 4.01128000 & -3.36729900 & 1.37088400 & $\mathrm{H}$ & -3.10213700 & -1.27339500 & -2.91851800 \\
\hline S & 2.90570700 & 0.57240600 & -1.33415200 & $\mathrm{C}$ & 0.09577700 & -2.08595900 & 0.17231300 \\
\hline $\mathrm{O}$ & 4.29461600 & 0.16410300 & -1.54654800 & $\mathrm{C}$ & 0.22293400 & -2.91517700 & -0.93560700 \\
\hline
\end{tabular}




\begin{tabular}{|c|c|c|c|c|c|c|}
\hline $\mathrm{C}$ & 0.09236900 & -2.56696500 & 1.47752500 & C $\quad 6.43994400$ & 0.52748700 & 0.17880800 \\
\hline $\mathrm{C}$ & 0.37791800 & -4.28968900 & -0.71646700 & C $\quad 5.36458900$ & 0.56668000 & 1.25289000 \\
\hline $\mathrm{H}$ & 0.20914500 & -2.52175900 & -1.94546100 & C 4.00221300 & -0.00635300 & 0.79854600 \\
\hline $\mathrm{C}$ & 0.24567500 & -3.94645300 & 1.67538800 & H $\quad 3.25022600$ & 1.85664100 & -0.11018700 \\
\hline $\mathrm{H}$ & -0.02374000 & -1.90539400 & 2.32914400 & H $\quad 6.54622800$ & -0.49317400 & -0.21631300 \\
\hline $\mathrm{C}$ & 0.38909100 & -4.80331900 & 0.58296600 & Н $\quad 7.40721100$ & 0.81962500 & 0.61425200 \\
\hline $\mathrm{H}$ & 0.48827400 & -4.95311000 & -1.57026300 & Н $\quad 5.70709600$ & -0.01938100 & 2.11341600 \\
\hline $\mathrm{H}$ & 0.24863200 & -4.33885900 & 2.68894900 & Н $\quad 5.23734500$ & 1.60101900 & 1.59745600 \\
\hline $\mathrm{H}$ & 0.50499900 & -5.87173400 & 0.74311100 & H $\quad 4.10976400$ & -1.05427500 & 0.49753700 \\
\hline $\mathrm{N}$ & 2.28719500 & -0.01097300 & -0.13465800 & H $\quad 3.33033200$ & 0.02300200 & 1.66824700 \\
\hline $\mathrm{S}$ & 3.10225900 & 0.08570200 & 1.31400400 & C 2.93290000 & 0.29232800 & -1.50011500 \\
\hline $\mathrm{O}$ & 4.23715600 & 1.01007000 & 1.27439500 & H $\quad 2.64074000$ & 0.97547100 & -2.29698400 \\
\hline $\mathrm{O}$ & 2.05877100 & 0.24481300 & 2.33309400 & H $\quad 3.13089500$ & -0.73972700 & -1.77977300 \\
\hline $\mathrm{C}$ & 3.85158600 & -1.64144800 & 1.61533600 & $\mathrm{Au} 0.77485700$ & 0.21076400 & -0.53287700 \\
\hline $\mathrm{F}$ & 3.32237600 & -2.53695700 & 0.78572500 & $\mathrm{P}-1.53137000$ & 0.36861300 & 0.13011500 \\
\hline $\mathrm{F}$ & 3.57880800 & -1.98076300 & 2.87289600 & C -2.72613200 & -0.53318000 & -0.89212600 \\
\hline $\mathrm{F}$ & 5.16548400 & -1.58500400 & 1.44063100 & C -3.60357000 & 0.15592900 & -1.74588000 \\
\hline $\mathrm{S}$ & 3.12621100 & -0.21550600 & -1.54856600 & C - 4.51868100 & -0.55935200 & -2.51909400 \\
\hline $\mathrm{O}$ & 4.46991400 & -0.74227300 & -1.29099500 & C -4.56481700 & -1.95282700 & -2.44559900 \\
\hline $\mathrm{O}$ & 2.23707400 & -0.85987600 & -2.51656100 & C -3.69568900 & -2.63963500 & -1.59224900 \\
\hline $\mathrm{C}$ & 3.38416000 & 1.51545000 & -2.23629700 & C -2.77401800 & -1.93808400 & -0.81808500 \\
\hline $\mathrm{F}$ & 3.79607600 & 2.34542800 & -1.28133700 & C -1.74388900 & -0.05209100 & 1.87828300 \\
\hline $\mathrm{F}$ & 2.23750500 & 1.95949000 & -2.75374100 & C -2.94281700 & -0.61619300 & 2.34390300 \\
\hline $\mathrm{F}$ & 4.30493800 & 1.43845000 & -3.19436800 & C -3.09552000 & -0.88089000 & 3.70571400 \\
\hline $\mathrm{C}$ & -0.48465900 & 2.73963600 & 0.79291000 & C -2.06387300 & -0.58709000 & 4.60002100 \\
\hline $\mathrm{O}$ & -0.92085800 & 5.60018500 & 0.56885000 & C -0.87171700 & -0.02067900 & 4.13785900 \\
\hline $\mathrm{C}$ & -0.10358500 & 5.68471800 & 1.74217200 & C -0.70658800 & 0.24536700 & 2.78011900 \\
\hline $\mathrm{C}$ & -0.40244300 & 4.48757300 & 2.63262000 & C -1.70502800 & 2.16188100 & -0.10234200 \\
\hline $\mathrm{C}$ & 0.12922100 & 3.13766900 & 2.09697700 & C - 1.34580400 & 2.72684300 & -1.34310600 \\
\hline $\mathrm{H}$ & -1.57269800 & 2.69455100 & 0.76633700 & C - 1.45030100 & 4.10373900 & -1.53427600 \\
\hline $\mathrm{H}$ & 0.96245600 & 5.70222400 & 1.47214400 & C - 1.90544300 & 4.92079000 & -0.49576600 \\
\hline $\mathrm{H}$ & -0.32220100 & 6.61343000 & 2.29060400 & C -2.25908400 & 4.36240000 & 0.73629600 \\
\hline $\mathrm{H}$ & 0.05700800 & 4.66204200 & 3.61266400 & C - -2.15650300 & 2.98717900 & 0.94187900 \\
\hline $\mathrm{H}$ & -1.48686900 & 4.42727700 & 2.79409300 & H -3.58693800 & 1.23839400 & -1.80371000 \\
\hline $\mathrm{H}$ & 1.22056200 & 3.17433600 & 2.00444800 & H -5.19838700 & -0.02276400 & -3.17461800 \\
\hline $\mathrm{H}$ & -0.11227300 & 2.37176100 & 2.84581100 & H -5.27990600 & -2.50440800 & -3.04949000 \\
\hline $\mathrm{C}$ & 0.21227900 & 2.46850000 & -0.34435600 & H -3.73322000 & -3.72320800 & -1.52913300 \\
\hline $\mathrm{H}$ & -0.29410700 & 2.38227000 & -1.30226700 & H - 2.10838200 & -2.47886600 & -0.15375700 \\
\hline $\mathrm{H}$ & 1.28582400 & 2.62195700 & -0.35733600 & H -3.75202600 & -0.84281100 & 1.65804900 \\
\hline \multirow[t]{2}{*}{$\mathrm{H}$} & -0.60036800 & 6.26203400 & -0.06622200 & H - 4.02309300 & -1.31696200 & 4.06491400 \\
\hline & & & & H -2.18737300 & -0.79958200 & 5.65827800 \\
\hline \multicolumn{2}{|c|}{ Int13 } & & & H - 0.06901800 & 0.20859400 & 4.83252500 \\
\hline $\mathrm{C}$ & 3.37033700 & 0.78881900 & -0.29655500 & H $\quad 0.22096000$ & 0.68315000 & 2.42135200 \\
\hline $\mathrm{O}$ & 6.07031800 & 1.43512500 & -0.86456500 & H -0.99560300 & 2.09133300 & -2.15273500 \\
\hline
\end{tabular}




\begin{tabular}{|c|c|c|c|c|c|c|}
\hline \multicolumn{2}{|c|}{ H - 1.17554100} & 4.53596200 & -2.49190200 & C $\quad 3.18638000$ & -4.44879100 & -0.98562900 \\
\hline \multicolumn{2}{|c|}{ H - 1.98447200} & 5.99367500 & -0.64653800 & C 2.15604700 & -3.87428900 & -1.73389900 \\
\hline \multicolumn{2}{|c|}{ H -2.61738000 } & 4.99808900 & 1.54077000 & C 1.67028200 & -2.61034200 & -1.39525600 \\
\hline \multicolumn{2}{|c|}{ H -2.43311300} & 2.56281500 & 1.90105800 & C $\quad 2.55903900$ & 0.99415800 & -0.76126600 \\
\hline $\mathrm{C}$ & 0.78292400 & -1.83152500 & -0.32008600 & C 2.31382000 & 2.35760500 & -0.51619000 \\
\hline $\mathrm{C}$ & 0.59671800 & -2.58155900 & -1.47292600 & C 3.05366600 & 3.33092500 & -1.18683000 \\
\hline $\mathrm{C}$ & 1.04768900 & -2.37040100 & 0.92895800 & C 4.03356300 & 2.95729000 & -2.11173100 \\
\hline $\mathrm{C}$ & 0.71036900 & -3.97379200 & -1.35708700 & C $\quad 4.27546700$ & 1.60535700 & -2.36159900 \\
\hline $\mathrm{H}$ & 0.37939600 & -2.12215000 & -2.43095400 & C 3.54304700 & 0.62336800 & -1.68992800 \\
\hline $\mathrm{C}$ & 1.15453200 & -3.76693000 & 1.01445900 & H 3.64962500 & 1.03512300 & 1.77484300 \\
\hline $\mathrm{H}$ & 1.16580500 & -1.75474000 & 1.81326900 & H $\quad 3.87899000$ & 1.28929600 & 4.22107000 \\
\hline $\mathrm{C}$ & 0.98650500 & -4.56148400 & -0.12074700 & H 2.12201100 & 0.40617900 & 5.74221600 \\
\hline $\mathrm{H}$ & 0.57714400 & -4.58677600 & -2.24427900 & H $\quad 0.12565400$ & -0.74011300 & 4.79939800 \\
\hline $\mathrm{H}$ & 1.36434400 & -4.21949300 & 1.97972400 & H -0.11833900 & -0.99196400 & 2.34930200 \\
\hline $\mathrm{H}$ & 1.06676500 & -5.64188800 & -0.04153800 & H $\quad 3.68165800$ & -1.96820500 & 1.29177200 \\
\hline \multirow[t]{2}{*}{$\mathrm{H}$} & 6.64900100 & 1.26759800 & -1.62701000 & H $\quad 4.53276100$ & -4.20533300 & 0.68388500 \\
\hline & & & & Н $\quad 3.56240400$ & -5.43425800 & -1.24667100 \\
\hline \multicolumn{2}{|c|}{ Int14 } & & & H $\quad 1.72792300$ & -4.40848300 & -2.57749200 \\
\hline \multicolumn{2}{|c|}{ C -3.67084500} & -1.24617400 & -0.15216100 & H $\quad 0.86716000$ & -2.16448800 & -1.98036600 \\
\hline \multicolumn{2}{|c|}{ O -5.07549900 } & -1.68161900 & -0.78458100 & H $\quad 1.55778200$ & 2.66080600 & 0.20210200 \\
\hline \multicolumn{2}{|c|}{ C -6.19822600} & -1.18298600 & 0.07461100 & H 2.86259500 & 4.38173600 & -0.98720500 \\
\hline \multicolumn{2}{|c|}{ C -5.51306200} & -0.94738700 & 1.40637500 & H $\quad 4.60636900$ & 3.71888800 & -2.63389000 \\
\hline \multicolumn{2}{|c|}{ C -4.13573500} & -0.39055000 & 1.015311 & H $\quad 5.03841600$ & 1.30886300 & -3.07607900 \\
\hline \multicolumn{2}{|c|}{ H -3.30296500 } & -2.22179300 & 0.16444000 & Н $\quad 3.74554800$ & -0.42407800 & -1.88879100 \\
\hline \multicolumn{2}{|c|}{ H -6.57183000 } & -0.26858400 & -0.38749700 & C -1.24528200 & 1.63092900 & -0.34341000 \\
\hline \multicolumn{2}{|c|}{ H -6.95195500} & -1.96975200 & 0.06350100 & C -1.28553800 & 2.42461500 & -1.48387200 \\
\hline \multicolumn{2}{|c|}{ H - 6.08872600} & -0.24738700 & 2.01755300 & C -1.40564000 & 2.13065000 & 0.94288900 \\
\hline \multicolumn{2}{|c|}{ H -5.41231900 } & -1.88910700 & 1.95574500 & C - 1.47312200 & 3.80296300 & -1.31259600 \\
\hline \multicolumn{2}{|c|}{ H -4.21427600} & 0.65763200 & 0.70855700 & H - 1.17313500 & 2.00494300 & -2.47753600 \\
\hline \multicolumn{2}{|c|}{ H -3.41641800 } & -0.46005800 & 1.83587700 & C - 1.59601200 & 3.51219000 & 1.08627800 \\
\hline \multicolumn{2}{|c|}{ C - 2.84792900} & -0.63020600 & -1.23491400 & H - 1.37418000 & 1.48733400 & 1.81495400 \\
\hline \multicolumn{2}{|c|}{ H - 2.68029800} & -1.32541900 & -2.06751600 & C - 1.62820400 & 4.34403100 & -0.03488000 \\
\hline \multicolumn{2}{|c|}{ H -3.27211600 } & 0.30823400 & -1.59665300 & H - 1.50022500 & 4.44376000 & -2.18983000 \\
\hline \multicolumn{2}{|c|}{$\mathrm{Au}-0.85514800$} & -0.35699500 & -0.57141800 & H - 1.71749500 & 3.92621900 & 2.08363900 \\
\hline \multicolumn{2}{|c|}{ P $\quad 1.54076700$} & -0.25817500 & 0.09844600 & H - 1.77788600 & 5.41305600 & 0.08727100 \\
\hline \multicolumn{2}{|c|}{ C 1.75116200} & -0.00009900 & 1.89417300 & H -5.15585300 & -1.35229900 & -1.70580600 \\
\hline $\mathrm{C}$ & 2.87708400 & 0.64474300 & 2.42965300 & & & \\
\hline $\mathrm{C}$ & 3.00571900 & 0.78815700 & 3.81280700 & Int15 & & \\
\hline $\mathrm{C}$ & 2.01877800 & 0.29010200 & 4.66676300 & C 3.27438800 & -0.35129500 & 0.52244000 \\
\hline $\mathrm{C}$ & 0.89661900 & -0.35416700 & 4.13826000 & O $\quad 6.25649300$ & -0.27850300 & 0.71414500 \\
\hline $\mathrm{C}$ & 0.75937700 & -0.49608000 & 2.75722800 & C $\quad 6.21422200$ & -0.99532900 & -0.52421000 \\
\hline $\mathrm{C}$ & 2.21845300 & -1.91028300 & -0.30535300 & C 4.99498400 & -1.90652800 & -0.53651900 \\
\hline $\mathrm{C}$ & 3.25206300 & -2.49410400 & 0.44450100 & C 3.63273300 & -1.18451800 & -0.67364800 \\
\hline $\mathrm{C}$ & 3.73207600 & -3.75871700 & 0.10086400 & Н 3.18988900 & -0.87852600 & 1.47303900 \\
\hline
\end{tabular}




\begin{tabular}{|c|c|c|c|c|c|c|}
\hline $\mathrm{H}$ & 6.17503000 & -0.29702100 & -1.37371200 & C $\quad 0.33458500$ & 2.60699800 & 0.00502200 \\
\hline $\mathrm{H}$ & 7.12324400 & -1.60507900 & -0.63995700 & C $\quad 0.56423700$ & 3.47921300 & 1.06931800 \\
\hline $\mathrm{H}$ & 5.09396500 & -2.59160000 & -1.38742600 & C -0.19468800 & 3.06002800 & -1.20209000 \\
\hline $\mathrm{H}$ & 5.00277800 & -2.52539700 & 0.37066400 & C $\quad 0.25797300$ & 4.83858000 & 0.91413800 \\
\hline $\mathrm{H}$ & 3.63102500 & -0.56368800 & -1.57796700 & H $\quad 0.97523800$ & 3.12605600 & 2.01129000 \\
\hline $\mathrm{H}$ & 2.86736700 & -1.96041200 & -0.80569900 & C -0.48946400 & 4.42343700 & -1.34798600 \\
\hline C & 3.13129400 & 1.01721000 & 0.52254700 & H - 0.38378900 & 2.37847400 & -2.02638300 \\
\hline $\mathrm{H}$ & 3.07270900 & 1.56030900 & 1.46318600 & C -0.26563000 & 5.31015700 & -0.29219300 \\
\hline $\mathrm{H}$ & 3.36978600 & 1.59610200 & -0.36745100 & H $\quad 0.43397300$ & 5.52357900 & 1.74036100 \\
\hline & 0.86072700 & 0.51336900 & 0.23572400 & H - 0.89717200 & 4.78425600 & -2.28952900 \\
\hline & 1.24079500 & -0.68112100 & 0.01721100 & H -0.49948900 & 6.36529100 & -0.40826700 \\
\hline & -2.43391500 & -0.33005600 & 1.35836700 & Н $\quad 6.97884000$ & 0.36863900 & 0.65475000 \\
\hline & -3.30921000 & -1.32233200 & 1.83059800 & & & \\
\hline & -4.21894800 & -1.02412000 & 2.84660900 & TS5 & & \\
\hline & -4.26226700 & 0.25911500 & 3.39762400 & C $\quad 3.28129300$ & 2.32516800 & -0.07862700 \\
\hline & -3.39343800 & 1.24882700 & 2.93072600 & O $\quad 3.80366000$ & 3.70343500 & -0.48693800 \\
\hline & -2.47917800 & 0.95754400 & 1.91751500 & C $\quad 5.18919400$ & 3.87643500 & -0.04883600 \\
\hline & -2.06493200 & -0.34190800 & -1.57756500 & C 5.33123800 & 2.88242300 & 1.09403100 \\
\hline & -3.45528100 & -0.18935200 & -1.68011800 & C $\quad 4.49638100$ & 1.69109800 & 0.60375400 \\
\hline & -4.03984900 & 0.05149600 & -2.92581800 & H $\quad 2.49113800$ & 2.56798600 & 0.63522600 \\
\hline & -3.24537000 & 0.13882800 & -4.07081400 & H $\quad 5.85427300$ & 3.64656800 & -0.88712900 \\
\hline & -1.85853700 & -0.01209400 & -3.97307700 & H $\quad 5.30263500$ & 4.92338400 & 0.23929000 \\
\hline & -1.26751500 & -0.24511100 & -2.73136100 & H $\quad 6.37730900$ & 2.62043900 & 1.27640100 \\
\hline & -0.91591500 & -2.48532700 & 0.07331600 & H $\quad 4.91114700$ & 3.30029500 & 2.01561900 \\
\hline & -0.07475000 & -2.97769000 & 1.08735300 & H $\quad 5.06482000$ & 1.10195700 & -0.12505200 \\
\hline & 0.19925100 & -4.34222600 & 1.17001600 & H $\quad 4.18284100$ & 1.02446300 & 1.41210600 \\
\hline & -0.35297000 & -5.22564800 & 0.23682400 & C $\quad 2.74934300$ & 1.61031700 & -1.25482600 \\
\hline & -1.18418500 & -4.74076500 & -0.77492000 & H $\quad 1.72960900$ & 1.77293700 & -1.58446400 \\
\hline & -1.46797300 & -3.37525800 & -0.86032700 & H $\quad 3.41425200$ & 1.02295200 & -1.88030600 \\
\hline & -3.28415600 & -2.32291200 & 1.41015400 & Au 0.58354100 & -1.05617900 & -0.24830400 \\
\hline & -4.89178000 & -1.79701600 & 3.20794600 & $\mathrm{P}-1.46136300$ & 0.21839800 & -0.30908000 \\
\hline & -4.96832300 & 0.48596400 & 4.19191800 & $\mathrm{C}-1.87718000$ & 1.00680700 & 1.29642100 \\
\hline & -3.42090100 & 2.24707800 & 3.35891100 & C -3.19482700 & 1.33574700 & 1.65421900 \\
\hline & -1.80011900 & 1.72815900 & 1.56576700 & C -3.45726500 & 1.94365300 & 2.88337700 \\
\hline & -4.08255900 & -0.25859400 & -0.79709200 & C -2.41079900 & 2.22804800 & 3.76522500 \\
\hline & -5.11739700 & 0.17096100 & -2.99800400 & C -1.09808700 & 1.90026500 & 3.41775800 \\
\hline & -3.70454200 & 0.32722900 & -5.03751200 & C -0.83175500 & 1.28843500 & 2.19127200 \\
\hline & -1.23688500 & 0.05903000 & -4.86132000 & C -1.40088300 & 1.57074500 & -1.55234000 \\
\hline & -0.18774500 & -0.35124900 & -2.65658200 & C -1.79149600 & 2.88745900 & -1.27004800 \\
\hline H & 0.36436100 & -2.29395900 & 1.81014200 & C - 1.67746100 & 3.87918200 & -2.24877000 \\
\hline & 0.84803800 & -4.71441600 & 1.95814200 & C - 1.17795900 & 3.56568100 & -3.51443000 \\
\hline & -0.13270600 & -6.28797900 & 0.29757500 & C -0.79134900 & 2.25299500 & -3.80446400 \\
\hline & -1.61584400 & -5.42393300 & -1.50135800 & C -0.89471400 & 1.26227800 & -2.82795100 \\
\hline & -2.11821200 & -3.01191600 & -1.64972500 & C -2.92444000 & -0.80005000 & -0.75487900 \\
\hline
\end{tabular}




\begin{tabular}{|c|c|c|c|c|c|c|}
\hline C - -2.97693900 & -2.12160500 & -0.28052600 & $\mathrm{~F}$ & 4.23897400 & 7.55208800 & -2.77988900 \\
\hline C -4.07593100 & -2.93129600 & -0.57076100 & $\mathrm{~F}$ & 3.74122500 & 7.00883300 & -0.72957100 \\
\hline C -5.12696800 & -2.43315200 & -1.34593900 & $\mathrm{~F}$ & 2.50626600 & 8.44585400 & -1.80698600 \\
\hline C -5.07702700 & -1.12293400 & -1.82796200 & & & & \\
\hline C -3.98176300 & -0.30671500 & -1.53480000 & $\mathrm{TS}$ & & & \\
\hline H -4.01743100 & 1.11823000 & 0.97942400 & & 56549100 & -1.10580000 & -0.09820700 \\
\hline H -4.48031500 & 2.19328000 & 3.15193200 & & -5.35787200 & -1.80545200 & -0.73524800 \\
\hline H -2.61893700 & 2.69942100 & 4.72214800 & & 1128300 & -1.04568100 & 0.05539600 \\
\hline H - 0.28132800 & 2.11325000 & 4.10221400 & & 5.55393300 & -0.67831500 & 1.32160200 \\
\hline H $\quad 0.18902500$ & 1.02213500 & 1.92883100 & & 4.18538000 & -0.14391600 & 0.86747700 \\
\hline H -2.17946700 & 3.14736100 & -0.29019300 & & .34089600 & -2.09406800 & 0.29609900 \\
\hline H - 1.97637600 & 4.89779700 & -2.01675600 & & -6.62281000 & -0.15801300 & -0.50227100 \\
\hline H - 1.08239100 & 4.34146800 & -4.26933400 & & -7.18083900 & -1.68097000 & 0.24870600 \\
\hline H - 0.39504900 & 2.00391700 & -4.78462400 & & -6.09912100 & 0.07148100 & 1.90250800 \\
\hline H - 0.57594000 & 0.24759600 & -3.05483600 & & -5.42387100 & -1.56709400 & 1.94994400 \\
\hline H -2.15545100 & -2.51615900 & 0.31279500 & & 28306700 & 0.84572900 & 0.40975500 \\
\hline H -4.10658700 & -3.95185100 & -0.19864600 & & -3.51341500 & -0.05636100 & 1.73149300 \\
\hline H -5.97968400 & -3.06581900 & -1.57786800 & & 2.84478400 & -0.69724600 & -1.26321500 \\
\hline H -5.89027000 & -0.73308800 & -2.43440200 & & -2.61031500 & -1.49702000 & -1.97162400 \\
\hline H -3.95386100 & 0.70970700 & -1.91588700 & & -3.17830700 & 0.22646400 & -1.73393600 \\
\hline C $\quad 2.31695500$ & -2.17263900 & -0.20564000 & & -0.80935800 & -0.37721000 & -0.52322400 \\
\hline C 3.03276200 & -2.47975200 & -1.38257000 & $\mathrm{P}$ & 1.56094500 & -0.24582600 & 0.10072200 \\
\hline C $\quad 2.85122400$ & -2.66 & 1.0 & $\mathrm{C}$ & 1.79 & 0 & 00 \\
\hline C $\quad 4.21260100$ & -3.23203700 & -1.35551100 & $\mathrm{C}$ & 2.92278500 & 0.84412700 & 2.31780700 \\
\hline H $\quad 2.66828700$ & -2.12560100 & -2.34516600 & $\mathrm{C}$ & 3.08721500 & 1.07634700 & 3.68480200 \\
\hline C $\quad 4.03075200$ & -3.41652400 & 1.04131800 & $\mathrm{C}$ & 2.13886700 & 0.60807000 & 4.59706700 \\
\hline H $\quad 2.34109900$ & -2.45757600 & 1.94413100 & $\mathrm{C}$ & 1.01913800 & -0.09727600 & 4.14555400 \\
\hline C $\quad 4.71760000$ & -3.70402700 & -0.14101100 & $\mathrm{C}$ & 0.84483900 & -0.33054200 & 2.78191300 \\
\hline H $\quad 4.73824000$ & -3.44908300 & -2.28358100 & $\mathrm{C}$ & 2.11849100 & -1.96014600 & -0.19019400 \\
\hline H $\quad 4.41287300$ & -3.77863900 & 1.99396900 & $\mathrm{C}$ & 2.95813000 & -2.62239200 & 0.71976000 \\
\hline H $\quad 5.63459100$ & -4.28842800 & -0.11642600 & $\mathrm{C}$ & 3.34174200 & -3.94154300 & 0.47463600 \\
\hline H $\quad 3.52316600$ & 4.13272200 & -1.63647900 & $\mathrm{C}$ & 2.89016700 & -4.60700400 & -0.66854700 \\
\hline $\mathrm{N} \quad 3.19239500$ & 4.68261300 & -2.69403000 & $\mathrm{C}$ & 2.05323700 & -3.95273100 & -1.57639600 \\
\hline S $\quad 4.04747500$ & 4.32042900 & -4.07145900 & $\mathrm{C}$ & 1.66470900 & -2.63390900 & -1.34021100 \\
\hline O 4.28537000 & 5.48813100 & -4.91722400 & $\mathrm{C}$ & 2.57488000 & 0.89180400 & -0.89926300 \\
\hline O $\quad 5.14577200$ & 3.45351200 & -3.64497800 & $\mathrm{C}$ & 2.36702300 & 2.27835100 & -0.78227900 \\
\hline C $\quad 2.87436900$ & 3.22115200 & -5.03544500 & $\mathrm{C}$ & 3.11784500 & 3.16102400 & -1.55732900 \\
\hline F $\quad 1.73998200$ & 3.87040200 & -5.28499000 & $\mathrm{C}$ & 4.06958300 & 2.67286300 & -2.45793800 \\
\hline F $\quad 2.61797700$ & 2.11573100 & -4.34069900 & $\mathrm{C}$ & 4.27548700 & 1.29735400 & -2.57795400 \\
\hline F $\quad 3.47346800$ & 2.90318200 & -6.18198800 & $\mathrm{C}$ & 3.53300400 & 0.40480700 & -1.80216200 \\
\hline S 2.14119200 & 5.96191000 & -2.53495500 & $\mathrm{H}$ & 3.66633400 & 1.20860000 & 1.61651800 \\
\hline O 1.60415300 & 6.39654800 & -3.82208400 & $\mathrm{H}$ & 3.95865400 & 1.62284200 & 4.03425100 \\
\hline $\mathrm{O} \quad 1.26261300$ & 5.63801600 & -1.41303300 & $\mathrm{H}$ & 2.27063000 & 0.79384800 & 5.65948800 \\
\hline C 3.24590600 & 7.34480100 & -1.91994500 & $\mathrm{H}$ & 0.28030900 & -0.46169700 & 4.85365800 \\
\hline
\end{tabular}




\begin{tabular}{|c|c|c|c|c|c|c|}
\hline H - 0.02699700 & -0.87810600 & 2.43188600 & $\mathrm{C}$ & 6.72808400 & -0.29098900 & 2.27064400 \\
\hline Н $\quad 3.31420500$ & -2.11473000 & 1.61037800 & $\mathrm{C}$ & 6.15394900 & -0.77083000 & 3.44980500 \\
\hline H $\quad 3.99464200$ & -4.44903200 & 1.17915400 & $\mathrm{C}$ & 4.80886600 & -1.15228400 & 3.46743700 \\
\hline H $\quad 3.18879000$ & -5.63561800 & -0.85078500 & $\mathrm{C}$ & 4.03896500 & -1.04904500 & 2.30908000 \\
\hline H $\quad 1.69994900$ & -4.46748100 & -2.46530300 & $\mathrm{C}$ & 3.45480000 & -2.16580600 & -1.07225300 \\
\hline H $\quad 1.01315300$ & -2.12560500 & -2.04854300 & $\mathrm{C}$ & 4.34395500 & -3.19256400 & -0.72078100 \\
\hline H $\quad 1.63357700$ & 2.66926500 & -0.08409600 & $\mathrm{C}$ & 4.19408200 & -4.46511300 & -1.27655700 \\
\hline H $\quad 2.95647000$ & 4.23080700 & -1.45900300 & $\mathrm{C}$ & 3.15955200 & -4.72247800 & -2.17996200 \\
\hline H $\quad 4.64979600$ & 3.36428300 & -3.06280500 & $\mathrm{C}$ & 2.26992200 & -3.70377300 & -2.53279800 \\
\hline Н $\quad 5.01712600$ & 0.91294700 & -3.27252000 & $\mathrm{C}$ & 2.41247000 & -2.43067700 & -1.97967500 \\
\hline H $\quad 3.70829100$ & -0.66135200 & -1.89964700 & $\mathrm{C}$ & 4.41245300 & 0.56777800 & -1.59953600 \\
\hline C -1.14164500 & 1.62681100 & -0.30583600 & $\mathrm{C}$ & 4.24873100 & 1.96194300 & -1.53783400 \\
\hline C -1.20424700 & 2.39668800 & -1.46022300 & $\mathrm{C}$ & 4.90242200 & 2.78382800 & -2.45654300 \\
\hline C -1.28620600 & 2.13960500 & 0.97509400 & $\mathrm{C}$ & 5.71583500 & 2.22318900 & -3.44514200 \\
\hline C -1.42302900 & 3.77281100 & -1.30793200 & $\mathrm{C}$ & 5.87910300 & 0.83743600 & -3.51174900 \\
\hline H - 1.09196100 & 1.96296900 & -2.44773600 & $\mathrm{C}$ & 5.23136000 & 0.00854300 & -2.59354300 \\
\hline C -1.50637300 & 3.51945700 & 1.09757800 & $\mathrm{H}$ & 6.42065400 & 0.18235700 & 0.19402800 \\
\hline H - 1.22201700 & 1.51277200 & 1.85722100 & $\mathrm{H}$ & 7.77273500 & 0.00730800 & 2.25380500 \\
\hline C -1.57355900 & 4.33046900 & -0.03672800 & $\mathrm{H}$ & 6.75102100 & -0.84510300 & 4.35468200 \\
\hline H - 1.47615700 & 4.39764800 & -2.19534100 & $\mathrm{H}$ & 4.35771000 & -1.52397500 & 4.38332100 \\
\hline H - 1.61977500 & 3.94754200 & 2.08985900 & $\mathrm{H}$ & 2.99185900 & -1.34205200 & 2.32920000 \\
\hline H - 1.74376600 & 5.39806100 & 0.06988700 & $\mathrm{H}$ & 5.15073400 & -3.00373700 & -0.01928400 \\
\hline \multirow[t]{2}{*}{ H -5.43610200 } & -1.56084700 & -1.67675200 & $\mathrm{H}$ & 4.88727800 & -5.25543200 & -1.00179900 \\
\hline & & & $\mathrm{H}$ & 3.04570200 & -5.71520700 & -2.60684900 \\
\hline TS4 & & & $\mathrm{H}$ & 1.46344500 & -3.89971300 & -3.23398300 \\
\hline C -1.29002300 & -0.77802700 & 1.67601100 & $\mathrm{H}$ & 1.71926600 & -1.64009100 & -2.26128900 \\
\hline O -2.79169900 & -0.89607000 & 1.73254200 & $\mathrm{H}$ & 3.61275600 & 2.40458000 & -0.77747400 \\
\hline C -3.29023300 & -0.59930000 & 3.08130100 & $\mathrm{H}$ & 4.77020200 & 3.86099600 & -2.40334700 \\
\hline C - -2.07607400 & -0.83307600 & 3.96551300 & $\mathrm{H}$ & 6.21899000 & 2.86487800 & -4.16344500 \\
\hline C -0.92377700 & -0.319 & 3.08957 & $\mathrm{H}$ & 6.51082500 & 0.39768300 & -4.27872800 \\
\hline H -0.95673800 & -1.80212800 & 1.48951700 & $\mathrm{H}$ & 5.36685600 & -1.06692300 & -2.65312200 \\
\hline H -3.63045400 & 0.43984900 & 3.10289100 & $\mathrm{C}$ & 1.29235000 & 1.96118500 & 0.80339100 \\
\hline H -4.12954400 & -1.27261900 & 3.26298100 & $\mathrm{C}$ & 0.78012000 & 2.96962000 & -0.00697100 \\
\hline H - 2.15869800 & -0.29747300 & 4.91520700 & $\mathrm{C}$ & 1.95331900 & 2.21432000 & 2.00081700 \\
\hline H - 1.95958800 & -1.90177600 & 4.17666700 & $\mathrm{C}$ & 0.97515600 & 4.29788500 & 0.39393800 \\
\hline H -0.87521100 & 0.77443100 & 3.12675600 & $\mathrm{H}$ & 0.24272400 & 2.74711000 & -0.92236400 \\
\hline H $\quad 0.04823400$ & -0.71818400 & 3.39298300 & $\mathrm{C}$ & 2.12883600 & 3.55096600 & 2.38436900 \\
\hline C -0.91327500 & 0.14335600 & 0.54053900 & $\mathrm{H}$ & 2.34124900 & 1.41301600 & 2.61992300 \\
\hline H - 1.29523100 & -0.21708000 & -0.42216200 & $\mathrm{C}$ & 1.64498200 & 4.58810800 & 1.58438900 \\
\hline H - 1.24418200 & 1.16580400 & 0.71982100 & $\mathrm{H}$ & 0.59033800 & 5.09936700 & -0.23111000 \\
\hline Au 1.15824700 & 0.02712700 & 0.17057600 & $\mathrm{H}$ & 2.64907400 & 3.76859900 & 3.31346000 \\
\hline P 3.55500600 & -0.47745600 & -0.36872300 & $\mathrm{H}$ & 1.78520100 & 5.62100100 & 1.89062100 \\
\hline C 4.61402400 & -0.57177600 & 1.11807300 & & 3.47385400 & -0.44304400 & 0.80828000 \\
\hline C $\quad 5.96380100$ & -0.19056200 & 1.10539900 & $\mathrm{~N}-2$ & 4.25472500 & -0.14512400 & -0.13196300 \\
\hline
\end{tabular}




\begin{tabular}{|c|c|c|c|c|c|}
\hline S - -4.83427300 & -1.44057200 & -0.99556200 & C 1.69902800 & -0.90390600 & -3.01153200 \\
\hline O -5.52511900 & -1.03303600 & -2.21624800 & C 1.30494200 & 1.45132600 & -0.39401000 \\
\hline O -3.75611500 & -2.42816900 & -1.02454500 & C $\quad 0.43802200$ & 2.33628700 & 0.27047200 \\
\hline C -6.13684000 & -2.15638700 & 0.14467200 & C $\quad 0.73343600$ & 3.69684400 & 0.32024000 \\
\hline F -7.06185000 & -1.23888000 & 0.41200200 & C 1.89092200 & 4.18362300 & -0.29588000 \\
\hline F -5.55402500 & -2.55294300 & 1.27553000 & C $\quad 2.75489900$ & 3.30665700 & -0.95568100 \\
\hline F -6.69568400 & -3.19991600 & -0.46397000 & C 2.46729500 & 1.94099800 & -1.00967200 \\
\hline S - 4.84641600 & 1.40098900 & -0.23761500 & H $\quad 2.64078300$ & 0.46021500 & 1.82360800 \\
\hline O -6.24769100 & 1.44836100 & -0.64954500 & H $\quad 3.03601800$ & -0.55593200 & 4.04165600 \\
\hline O -4.38592000 & 2.09327400 & 0.96618700 & H $\quad 1.80339600$ & -2.60661800 & 4.71604900 \\
\hline C -3.86874900 & 2.14297800 & -1.65462000 & H $\quad 0.16572100$ & -3.64291800 & 3.15742900 \\
\hline F - 4.02768000 & 1.41209300 & -2.75433700 & H -0.25027300 & -2.62443300 & 0.93686600 \\
\hline F - 2.57721100 & 2.19313000 & -1.33170100 & H $\quad 3.06468400$ & -2.31961500 & -0.21173700 \\
\hline \multirow[t]{2}{*}{ F - -4.32815000} & 3.37581900 & -1.85987700 & H $\quad 4.41340800$ & -3.46592700 & -1.93486500 \\
\hline & & & H $\quad 4.03785500$ & -2.98407500 & -4.34478900 \\
\hline TS6 & & & H $\quad 2.30146800$ & -1.33901400 & -5.02846100 \\
\hline C -4.36715400 & -0.06926900 & -0.44225400 & Н $\quad 0.93545600$ & -0.19231200 & -3.31511500 \\
\hline O -5.46019700 & 0.73728000 & 0.22010700 & H - 0.46415300 & 1.96287800 & 0.74400600 \\
\hline C -6.28447200 & -0.11199400 & 1.08685400 & H $\quad 0.05263000$ & 4.37399100 & 0.82818600 \\
\hline C -6.11033300 & -1.49199900 & 0.47598500 & H $\quad 2.11721600$ & 5.24584000 & -0.26349600 \\
\hline C -4.62810200 & -1.49062300 & 0.06921800 & H $\quad 3.65559200$ & 3.68292600 & -1.43254400 \\
\hline H -4.58164600 & 0.03016400 & -1.50764900 & H $\quad 3.14501700$ & 1.26759500 & -1.52418500 \\
\hline H -5.89785600 & -0.05042900 & 2.10804100 & H -5.22938700 & 1.86704100 & 0.60914500 \\
\hline H -7.29840500 & 0.28981800 & 1.04289000 & N -5.10447100 & 3.10614000 & 0.88295000 \\
\hline H -6.35182900 & -2.28414500 & 1.18989600 & S -5.50295700 & 4.13127700 & -0.36072800 \\
\hline H -6.75679600 & -1.60169000 & -0.40137700 & O -5.07087500 & 5.50600800 & -0.12102800 \\
\hline H -3.99426800 & -1.69280700 & 0.94017000 & O -5.15054500 & 3.43093700 & -1.59593100 \\
\hline H -4.39908100 & -2.23044100 & -0.70285900 & C -7.37454100 & 4.13231900 & -0.29898600 \\
\hline C -3.03848200 & 0.54213800 & -0.08241200 & F - 7.78482500 & 4.61029900 & 0.87303800 \\
\hline H -2.88426700 & 1.54074500 & -0.49366900 & F - 7.81497100 & 2.88508700 & -0.45842900 \\
\hline H -2.81700600 & 0.49320200 & 0.98441800 & F - 7.82857600 & 4.90114900 & -1.28590000 \\
\hline $\mathrm{Au}-1.44233900$ & -0.58519600 & -1.00726800 & S -4.82562200 & 3.58091500 & 2.44506600 \\
\hline $\mathrm{P} \quad 0.87773300$ & -0.31692200 & -0.42053100 & O -5.45171300 & 4.85896300 & 2.77658300 \\
\hline C $\quad 1.17572000$ & -1.02246700 & 1.22910600 & O -5.03406400 & 2.39723100 & 3.27919500 \\
\hline C $\quad 2.09873700$ & -0.43573800 & 2.10986700 & C - 2.97811700 & 3.89228400 & 2.46182400 \\
\hline C $\quad 2.32197100$ & -1.01172000 & 3.36155500 & F - 2.64549300 & 4.72893800 & 1.48033200 \\
\hline C $\quad 1.62964900$ & -2.16514700 & 3.73852700 & F -2.33388500 & 2.73531700 & 2.30214700 \\
\hline C $\quad 0.70875600$ & -2.74925200 & 2.86331600 & F -2.65558400 & 4.42389700 & 3.63792300 \\
\hline C $\quad 0.47619200$ & -2.17923100 & 1.61223800 & & & \\
\hline C $\quad 1.91549800$ & -1.17176000 & -1.64640200 & Int17 & & \\
\hline C $\quad 2.89202000$ & -2.10433800 & -1.26108000 & C $\quad 3.20871500$ & 0.73569700 & 0.45732500 \\
\hline C 3.65411300 & -2.74987500 & -2.23622800 & O $\quad 6.01718300$ & 1.47819900 & 0.58386500 \\
\hline C $\quad 3.44425100$ & -2.47587600 & -3.58991000 & C $\quad 6.26601600$ & 0.08088000 & 0.77041300 \\
\hline C $\quad 2.46817100$ & -1.55105700 & -3.97618700 & C $\quad 5.12087900$ & -0.51596100 & 1.57506800 \\
\hline
\end{tabular}




\begin{tabular}{|c|c|c|c|c|c|}
\hline C $\quad 3.77407000$ & -0.60792000 & 0.82113300 & H -3.06853600 & -3.43624600 & 3.03637900 \\
\hline H $\quad 3.05843700$ & 1.43755700 & 1.27783500 & H -2.61393600 & -2.69791600 & 0.72046700 \\
\hline Н $\quad 6.35647200$ & -0.43009700 & -0.19970900 & H $\quad 6.68427800$ & 1.81979700 & -0.03455100 \\
\hline Н $\quad 7.21138700$ & -0.06821600 & 1.31350600 & & & \\
\hline H $\quad 5.40320900$ & -1.53283800 & 1.87280400 & Int18 & & \\
\hline H $\quad 4.99360700$ & 0.06309700 & 2.49904200 & $\mathrm{Au} 0.10836800$ & -0.10439800 & -0.11898700 \\
\hline Н $\quad 3.88719800$ & -1.22273600 & -0.07985700 & P -2.19774500 & -0.54865400 & -0.19376200 \\
\hline H 3.06362100 & -1.11878000 & 1.48322900 & C -2.92053000 & -0.51887500 & 1.47791900 \\
\hline C $\quad 2.98163300$ & 1.18315000 & -0.82526800 & C -4.20036600 & -1.05908100 & 1.69657900 \\
\hline H 2.75661500 & 2.23190500 & -1.00926600 & C -4.74612400 & -1.04746800 & 2.97955000 \\
\hline Н 3.26109500 & 0.57346400 & -1.68299100 & C -4.01993900 & -0.51151100 & 4.04791100 \\
\hline $\mathrm{Au} 0.86770700$ & 0.42860300 & -0.28637400 & C -2.74352200 & 0.01301800 & 3.83525500 \\
\hline P -1.42752500 & -0.19379400 & -0.32609400 & C -2.19037200 & 0.01023200 & 2.55318100 \\
\hline C -2.32381300 & 1.31128100 & -0.73719800 & C -3.11834400 & 0.56344400 & -1.29885600 \\
\hline C -3.35627600 & 1.81116900 & 0.08196600 & C - -4.18950400 & 1.34557100 & -0.84130900 \\
\hline C -3.99533200 & 2.99336900 & -0.27439000 & C -4.85076500 & 2.20094900 & -1.72583200 \\
\hline C -3.60618000 & 3.68789000 & -1.42896100 & C -4.44750800 & 2.28251700 & -3.05973700 \\
\hline C -2.57647100 & 3.19795000 & -2.24071800 & C -3.37498800 & 1.50918000 & -3.51677300 \\
\hline C -1.92691000 & 2.01793600 & -1.89873300 & C -2.70638600 & 0.65539500 & -2.64165100 \\
\hline C -1.60386200 & -1.47151900 & -1.58076800 & C -2.35414400 & -2.25292000 & -0.82771300 \\
\hline C - 2.61412600 & -1.40791200 & -2.56101000 & C -1.46783500 & -3.22447700 & -0.32677900 \\
\hline C -2.69780500 & -2.41401600 & -3.51811600 & C -1.56550700 & -4.54526200 & -0.76101300 \\
\hline C -1.77665700 & -3.47037700 & -3.51561600 & C -2.54234400 & -4.90422300 & -1.69497500 \\
\hline C -0.76939400 & -3.53280800 & -2.54612100 & C -3.42444900 & -3.94155900 & -2.19217200 \\
\hline C -0.67314700 & -2.53701000 & -1.58054600 & C -3.33418300 & -2.61556100 & -1.76452500 \\
\hline C -1.78937700 & -0.78453800 & 1.33354400 & H -4.76584600 & -1.48714200 & 0.87387800 \\
\hline C -1.45410100 & 0.05196600 & 2.42629900 & H -5.73633000 & -1.46245400 & 3.14518200 \\
\hline C -1.71461300 & -0.37629200 & 3.72260400 & H -4.44776000 & -0.51010000 & 5.04664600 \\
\hline C - 2.28931900 & -1.63403900 & 3.93978300 & H -2.17381900 & 0.42054200 & 4.66556500 \\
\hline C -2.61051200 & -2.46797300 & 2.85866400 & H - 1.19100500 & 0.40386500 & 2.39229100 \\
\hline C -2.35654100 & -2.05554200 & 1.55558600 & H -4.51298400 & 1.29017400 & 0.19280300 \\
\hline H -3.66856200 & 1.27251800 & 0.96982300 & H -5.68192700 & 2.80186200 & -1.36794600 \\
\hline H -4.80491400 & 3.37350400 & 0.34110100 & H -4.96482400 & 2.94969300 & -3.74366300 \\
\hline H -4.11036300 & 4.61212500 & -1.69591800 & H -3.05701900 & 1.57313800 & -4.55345900 \\
\hline H - 2.27849300 & 3.73913100 & -3.13336100 & H - 1.86948700 & 0.06165600 & -3.00045100 \\
\hline H -1.12113000 & 1.63768600 & -2.51983200 & H -0.70750100 & -2.94750200 & 0.39966500 \\
\hline H -3.33340000 & -0.59564300 & -2.56269500 & H -0.87814500 & -5.29170200 & -0.37318100 \\
\hline H -3.48476600 & -2.38062600 & -4.26562000 & H -2.61514500 & -5.93362700 & -2.03481700 \\
\hline H - 1.84662700 & -4.24762100 & -4.27125100 & H -4.18635800 & -4.22057000 & -2.91449500 \\
\hline H -0.05806700 & -4.35305500 & -2.54752100 & H -4.02415900 & -1.87568400 & -2.15668700 \\
\hline H $\quad 0.11016300$ & -2.57835600 & -0.82896500 & $\mathrm{~N} \quad 2.31902400$ & -0.23696600 & -0.05706300 \\
\hline H - 1.00267000 & 1.02426700 & 2.25218000 & S 3.05953600 & -0.27578400 & 1.42756000 \\
\hline H - 1.46630800 & 0.26404900 & 4.56331300 & O 4.51130300 & -0.11255500 & 1.35762800 \\
\hline H - 2.48901900 & -1.96755100 & 4.95395700 & O $\quad 2.26139700$ & 0.56423400 & 2.32448500 \\
\hline
\end{tabular}




\begin{tabular}{|c|c|c|c|c|c|c|}
\hline $\mathrm{C}$ & 2.74844600 & -2.03279500 & 2.00771800 & $\mathrm{Au} 1.05248300$ & 0.55369900 & -0.16274500 \\
\hline$F$ & 1.43637900 & -2.21268300 & 2.18568600 & $\mathrm{P}-1.22164700$ & -0.06147000 & 0.00592900 \\
\hline $\mathrm{F}$ & 3.38351300 & -2.20920900 & 3.16398400 & C -1.71739500 & -1.19979300 & -1.33528900 \\
\hline $\mathrm{F}$ & 3.19762300 & -2.90199900 & 1.10619600 & C - 2.95502200 & -1.08193500 & -1.98611800 \\
\hline S & 3.12044900 & -0.63218500 & -1.45733100 & C -3.30016200 & -1.98755200 & -2.99217000 \\
\hline $\mathrm{O}$ & 4.30303300 & -1.46689200 & -1.24294400 & C -2.41859600 & -3.00915500 & -3.35185100 \\
\hline $\mathrm{O}$ & 2.10004800 & -1.03123500 & -2.43130100 & C -1.18395900 & -3.12821100 & -2.70585400 \\
\hline $\mathrm{C}$ & 3.76030200 & 1.02579300 & -2.05767400 & C -0.82956500 & -2.22428600 & -1.70521500 \\
\hline $\mathrm{F}$ & 4.40426100 & 1.64967100 & -1.07419900 & C -1.55621600 & -0.90277500 & 1.59346700 \\
\hline $\mathrm{F}$ & 2.73650600 & 1.77553700 & -2.47010900 & C -2.49717800 & -1.93993100 & 1.69027600 \\
\hline $\mathrm{F}$ & 4.58925600 & 0.80648000 & -3.07550000 & C - 2.74431900 & -2.54144600 & 2.92611400 \\
\hline & 0.56788900 & 2.74955500 & 0.62106100 & C -2.05826400 & -2.11497500 & 4.06563900 \\
\hline & -0.92187100 & 5.23625200 & 0.05877700 & C - 1.11946400 & -1.08311300 & 3.97241300 \\
\hline & -0.10365000 & 5.64133800 & 1.16518400 & C -0.86411200 & -0.48054500 & 2.74072700 \\
\hline & -0.29510200 & 4.62201300 & 2.27640800 & C -2.30315300 & 1.40882900 & -0.08350700 \\
\hline $\mathrm{C}$ & 0.16514200 & 3.21677900 & 1.82499400 & C - 1.96138900 & 2.43943500 & -0.97509200 \\
\hline & -1.65274500 & 2.70249400 & 0.68755500 & C -2.77511600 & 3.56639800 & -1.08595000 \\
\hline $\mathrm{H}$ & 0.94879300 & 5.69560300 & 0.86308500 & C -3.92758000 & 3.67755600 & -0.30198300 \\
\hline & -0.41472600 & 6.63921300 & 1.50598300 & C -4.26752600 & 2.65761300 & 0.58962000 \\
\hline $\mathrm{H}$ & 0.27796800 & 4.92179300 & 3.16070300 & C -3.46037700 & 1.52326400 & 0.70218000 \\
\hline & 1.35359600 & 4.59350900 & 2.56319200 & H -3.64867500 & -0.29311000 & -1.71306200 \\
\hline $\mathrm{H}$ & 1.24525600 & 3.20202300 & 1.64707400 & H -4.25883200 & -1.89107800 & -3.49435500 \\
\hline & -0.05128700 & 2.51619800 & 2.64560000 & H -2.68965000 & -3.70878700 & -4.13781500 \\
\hline $\mathrm{C}$ & 0.03395400 & 2.30091500 & -0.54781300 & H -0.49390400 & -3.91904100 & -2.98655200 \\
\hline & -0.56677000 & 2.16065100 & -1.44245000 & H $\quad 0.13615000$ & -2.31004200 & -1.21322100 \\
\hline $\mathrm{H}$ & 1.10414700 & 2.42765300 & -0.68209200 & H - 3.03625700 & -2.27787100 & 0.81069000 \\
\hline \multirow{2}{*}{\multicolumn{2}{|c|}{ H - 0.45804900}} & 5.44582600 & -0.76914400 & H -3.47285500 & -3.34457700 & 2.99524100 \\
\hline & & & & H -2.24990000 & -2.58974100 & 5.02411400 \\
\hline \multicolumn{2}{|c|}{ Int19 } & & & H -0.57986600 & -0.75314000 & 4.85580300 \\
\hline$C$ & 4.04131700 & 0.37554900 & 0.55422500 & H -0.12407800 & 0.31268500 & 2.66724900 \\
\hline $\mathrm{O}$ & 5.36238500 & 0.96077700 & 0.33977400 & H - 1.05801300 & 2.36074000 & -1.57489000 \\
\hline C & 6.16595500 & 0.04786500 & -0.43876700 & H -2.50454500 & 4.36040500 & -1.77637400 \\
\hline $\mathrm{C}$ & 5.63453600 & -1.33317500 & -0.07898000 & H -4.55585900 & 4.56039800 & -0.38293700 \\
\hline $\mathrm{C}$ & 4.12116200 & -1.07035400 & 0.02339200 & H - 5.16149900 & 2.74243000 & 1.20123500 \\
\hline $\mathrm{H}$ & 3.84670300 & 0.42025700 & 1.62834800 & H -3.73481200 & 0.73548700 & 1.39665000 \\
\hline $\mathrm{H}$ & 6.05460700 & 0.26784800 & -1.50891000 & & & \\
\hline $\mathrm{H}$ & 7.20620000 & 0.23096800 & -0.15226000 & Int20 & & \\
\hline $\mathrm{H}$ & 5.88378800 & -2.08891100 & -0.82944800 & $\mathrm{Au} 0.14821900$ & -0.25983800 & -1.99129800 \\
\hline $\mathrm{H}$ & 6.04042800 & -1.65267300 & 0.88725500 & P -0.08807200 & -0.10652200 & 0.30197400 \\
\hline $\mathrm{H}$ & 3.65751300 & -1.13519300 & -0.96701600 & C $\quad 1.55085300$ & 0.33818600 & 0.89144300 \\
\hline $\mathrm{H}$ & 3.60668500 & -1.77097700 & 0.68647800 & C $\quad 2.20751300$ & -0.42139700 & 1.87896800 \\
\hline $\mathrm{C}$ & 3.15958500 & 1.32861800 & -0.20647100 & C 3.48100900 & -0.04252600 & 2.29078100 \\
\hline $\mathrm{H}$ & 2.98644000 & 2.30394800 & 0.25393700 & C 4.10694500 & 1.07333300 & 1.71809700 \\
\hline $\mathrm{H}$ & 3.33068800 & 1.36627300 & -1.28530000 & C 3.45789700 & 1.82558000 & 0.73250400 \\
\hline
\end{tabular}




\begin{tabular}{|c|c|c|c|c|c|}
\hline C $\quad 2.18369300$ & 1.46324400 & 0.31161300 & H-2.14022700 & 1.15799400 & 0.82556200 \\
\hline C -1.31923400 & 1.16220500 & 0.61909200 & H-2.46867700 & 1.10731800 & -0.94590200 \\
\hline C -1.06218200 & 2.20650100 & 1.52835000 & O-2.14771400 & -0.73534700 & -0.09458200 \\
\hline C -2.04574900 & 3.16345200 & 1.75797100 & H-1.14114500 & -0.75746100 & -0.34353600 \\
\hline C -3.26994400 & 3.09374100 & 1.08051700 & $\mathrm{H}-2.25693100$ & -1.22452900 & 0.74931600 \\
\hline C -3.52264700 & 2.06065800 & 0.17057900 & В 1.13177500 & 0.05553000 & 0.02733900 \\
\hline C -2.55246400 & 1.09405800 & -0.06941500 & F 1.82190700 & 0.86699400 & -0.85929000 \\
\hline C -0.60514700 & -1.74829700 & 0.81890100 & F 0.30861900 & 0.80486900 & 0.87060500 \\
\hline C $\quad 0.15022700$ & -2.85696000 & 0.36898900 & F 0.24503600 & -0.80911000 & -0.76216600 \\
\hline C -0.23067600 & -4.14175000 & 0.73842600 & F 1.98431400 & -0.76375200 & 0.75106900 \\
\hline C -1.35916600 & -4.33012800 & 1.54414100 & & & \\
\hline C -2.10867000 & -3.23219000 & 1.98820000 & $\mathrm{MeOH}_{2}^{+}$ & & \\
\hline C -1.74405400 & -1.93979600 & 1.62465600 & C 0.77555600 & -0.00185400 & 0.05668500 \\
\hline H 1.72402400 & -1.28261900 & 2.32702000 & H 1.05924200 & 0.36164300 & -0.92811800 \\
\hline H 3.98699100 & -0.61329300 & 3.06339600 & H 1.13965900 & 0.64473000 & 0.85155900 \\
\hline H $\quad 5.10354100$ & 1.35677400 & 2.04390900 & H 1.04222100 & -1.04649000 & 0.21079000 \\
\hline H 3.94647200 & 2.68914800 & 0.29211700 & O- 0.71019500 & 0.12284200 & 0.10285200 \\
\hline H $\quad 1.67480800$ & 2.03982300 & -0.45498800 & H-1.14079400 & -0.40181800 & -0.61061000 \\
\hline H -0.11785600 & 2.25859000 & 2.05932500 & H-1.07498200 & -0.19913500 & 0.95893900 \\
\hline H - 1.86161500 & 3.96245600 & 2.46957800 & & & \\
\hline H -4.02999500 & 3.84729400 & 1.26509500 & $\mathrm{BF}_{4}^{-}$ & & \\
\hline H -4.47291000 & 2.01247900 & -0.35192600 & B 0.00000000 & 0.00000000 & 0.00000000 \\
\hline H -2.74111800 & 0.29191600 & -0.77688800 & F 0.81233300 & 0.81233300 & 0.81233300 \\
\hline H $\quad 1.02475600$ & -2.70397900 & -0.25633200 & F- 0.81233300 & 0.81233300 & -0.81233300 \\
\hline H $\quad 0.34734500$ & -4.99492100 & 0.39712700 & F 0.81233300 & -0.81233300 & -0.81233300 \\
\hline H - 1.65590000 & -5.33508600 & 1.82994000 & F- 0.81233300 & -0.81233300 & 0.81233300 \\
\hline H -2.97630600 & -3.38668000 & 2.62223400 & & & \\
\hline \multirow[t]{2}{*}{ H - 2.32465600} & -1.09315400 & 1.97473000 & $\mathrm{PF}_{6}^{-}$ & & \\
\hline & & & P 0.00000000 & 0.00000000 & 0.00000000 \\
\hline $\mathrm{N}_{2}$ & & & F 0.00000000 & 0.00000000 & 1.63577700 \\
\hline N 0.00000000 & 0.00000000 & 0.55240300 & F 0.00000000 & 1.63577700 & 0.00000000 \\
\hline \multirow[t]{2}{*}{ N 0.00000000} & 0.00000000 & -0.55240300 & F-1.63577700 & 0.00000000 & 0.00000000 \\
\hline & & & F 0.00000000 & 0.00000000 & -1.63577700 \\
\hline $\mathrm{MeOH}$ & & & F 0.00000000 & -1.63577700 & 0.00000000 \\
\hline C -0.04812700 & 0.66583000 & 0.00000000 & F 1.63577700 & 0.00000000 & 0.00000000 \\
\hline H - 1.09398300 & 0.98832200 & 0.00000000 & & & \\
\hline H $\quad 0.44392100$ & 1.07788400 & 0.89291100 & $\mathrm{HBF}_{4}$ & & \\
\hline H $\quad 0.44392100$ & 1.07788400 & -0.89291100 & B- 0.41653500 & 0.03772500 & -0.01080500 \\
\hline $\mathrm{O}-0.04812700$ & -0.76141400 & 0.00000000 & F- 0.62565500 & -0.13989300 & 1.29014700 \\
\hline \multirow[t]{2}{*}{ H $\quad 0.87992200$} & -1.04775600 & 0.00000000 & F 1.61774500 & -0.25781400 & 0.05407500 \\
\hline & & & F-0.65390200 & -0.96655200 & -0.84853000 \\
\hline $\mathrm{MeOH}_{2}-\mathrm{BF}_{4}$ & & & F-0.33422600 & 1.27811800 & -0.48913600 \\
\hline C-2.66304200 & 0.64587100 & 0.02047100 & Н 2.04701900 & 0.58664700 & -0.00496800 \\
\hline H-3.73081000 & 0.55556200 & 0.20972400 & & & \\
\hline
\end{tabular}


$\mathrm{HPF}_{6}$

$\begin{array}{lrcc}\text { P } & 0.24811200 & -0.00414900 & 0.00004200 \\ \text { F }-0.09523800 & -1.26172500 & -0.92666400 \\ \text { F }-0.05021300 & -0.91889800 & 1.27535200 \\ \text { F }-1.96270700 & 0.15797900 & 0.01149500 \\ \text { F } & 0.07921500 & 1.28184600 & 0.92838000 \\ \text { F } & 0.06429800 & 0.93648900 & -1.27583200 \\ \text { F } & 1.81387800 & -0.11072700 & -0.00561100 \\ \text { H }-2.36476400 & -0.70243700 & -0.06469800\end{array}$

Int15'

$\begin{array}{llll}\text { C } & 1.94391300 & 1.84724200 & -0.10854500\end{array}$

$\begin{array}{llll}\text { O } & 3.04067400 & 4.23812200 & -1.10973600\end{array}$

C $\quad 4.08529500 \quad 4.08262600 \quad-0.14355000$

$\begin{array}{llll}\text { C } & 3.49103200 & 3.45125400 & 1.10541100\end{array}$

$\begin{array}{llll}\text { C } & 3.03366900 & 1.98847600 & 0.89885900\end{array}$

$\begin{array}{llll}\mathrm{H} & 1.04007900 & 2.43096700 & 0.05911500\end{array}$

$\begin{array}{llll}\mathrm{H} & 4.89180000 & 3.45202600 & -0.54339600\end{array}$

$\begin{array}{llll}\mathrm{H} & 4.52000000 & 5.06223700 & 0.10656300\end{array}$

$\mathrm{H} \quad 4.24423800 \quad 3.46661800 \quad 1.90185300$

H $\quad 2.64623700 \quad 4.06306300 \quad 1.44749100$

$\begin{array}{llll}\mathrm{H} & 3.88796300 & 1.35796200 & 0.62688500\end{array}$

$\mathrm{H} \quad 2.65256400 \quad 1.62622800 \quad 1.86572600$

$\begin{array}{llll}\text { C } & 1.98728300 & 1.02239500 & -1.21151500\end{array}$

$\begin{array}{llll}\mathrm{H} & 1.21733400 & 1.09530900 & -1.97563400\end{array}$

$\begin{array}{llll}\mathrm{H} & 2.91091400 & 0.52276300 & -1.48741200\end{array}$

$\mathrm{Au} \quad 0.76844600 \quad-0.82123500 \quad-0.10096400$

P -1.56250100

C -2.01801800

C $\quad-3.35696100$

C -3.65929400

C -2.63254800

C -1.29962400

C -0.99189300

C -1.93946900

C -2.38989700

C -2.61698700

C -2.39912200

C -1.94808700

C -1.71249200

C -2.73798000

C -2.38532900

C $\quad-3.25583600$

C -4.48097600

C -4.83483400
$-0.01974500$

0.49908100

0.58845600

0.98412700

1.29167900

1.20227500

0.80473200

1.38924700

2.62850200

3.68203200

3.50703100

2.27344800

1.22140200

$-1.35207500$

$-2.67675700$

$-3.72531100$

$-3.46109200$

$-2.14641400-1.35093300$
0.03481600

1.73365500

2.15034000

3.45376000
C $\quad-3.96947200$

$-1.09308200$

$-1.04674500$

H -4.16329200

0.34741500

1.46382800

H -4.69726000

1.04854500

3.76877800

H $\quad-2.87237000$

1.59527000

5.36728600

H $\quad-0.49816700$

1.43578400

4.64073100

H 0.04722100

H $\quad-2.56915400$

0.72746300

2.33148600

H $\quad-2.96742900$

2.77761000

0.45692300

H -2.57828500

H $\quad-1.77593100$

4.63801200

$-1.11405300$

4.32716800

$-3.55105100$

H $\quad-1.35973700$

2.13106600

$-4.40546300$

H -1.42918600

0.26673200

$-2.84051100$

H $\quad-2.97401700$

$-2.88633900$

0.35716600

H $\quad-5.15585700$

$-4.74646900$

$-0.17648500$

H $\quad-5.78521900$

$-4.27826200$

$-1.27719600$

H $\quad-4.25380800$

$-1.93807100$

$-1.83501400$

C 2.56501800

$-0.07597400$

$-1.29836000$

C 3.04970200

$-1.83451900$

$-0.08555300$

C 3.25941800

$-2.46044700$

$-1.24405900$

C 4.18272900

$-1.99321700$

1.12315300

H 2.54683600

$-3.27965000$

$-1.18089100$

C $\quad 4.39337900$

$-2.32133500$

$-2.19826100$

H 2.91934700

$-2.81188500$

1.18252300

C 4.85547800

$-1.49203000$

2.02642600

H 4.54185200

$-3.45580300$

0.03146800

H 4.91627700

$-3.77305900$

$-2.08117800$

H $\quad 5.74259400$

$-2.94009300$

2.12751900

H 3.45080500

$-4.08257200$

0.07667900

$\mathrm{TS}^{\prime}$

C 1.39701300

$-0.62794400 \quad-1.49806400$

O 2.81343000

$-0.30180900$

$-1.93229700$

C 3.24894300

$-1.20569600$

$-2.99327200$

C 2.22372200

$-2.33387800$

$-2.99548000$

C 0.93684700

$-1.61098500$

$-2.57545200$

H 1.51707500

$-1.14029900$

$-0.53889500$

H 3.24669700

$-0.64053500$

$-3.93106600$

H 4.27044200

$-1.52160400$

$-2.76749200$

H 2.14783700

$-2.81767300$

$-3.97383700$

H 2.49656200

$-3.09417300$

$-2.25434900$

H 0.50921700

$-1.06046500$

$-3.42303300$

H $\quad 0.17352800$

$-2.29273000$

$-2.18966600$

C $\quad 0.62605900$

0.65657800

$-1.34069400$

H 1.13570900

1.32133300

$-0.63495000$ 


\begin{tabular}{|c|c|c|c|c|c|c|c|}
\hline $\mathrm{H}$ & 0.53811400 & 1.17621000 & -2.30273400 & $\mathrm{H}$ & -0.61402400 & 5.46624600 & 0.80584500 \\
\hline & -1.32862100 & 0.33132800 & -0.55405700 & $\mathrm{H}$ & -3.93298300 & 4.84915900 & -1.86833500 \\
\hline $\mathrm{P}$ & -3.40042000 & -0.66368200 & 0.32792200 & $\mathrm{H}$ & -2.51167000 & 6.38370900 & -0.51912700 \\
\hline $\mathrm{C}$ & -4.88954700 & -0.21809700 & -0.65098000 & $\mathrm{~N}$ & 4.45770700 & -0.02061600 & -0.04629600 \\
\hline$C$ & -6.11818200 & 0.11698900 & -0.06403200 & $\mathrm{H}$ & 3.67734100 & -0.19853400 & -0.90477200 \\
\hline $\mathrm{C}$ & -7.21188100 & 0.45267400 & -0.86718800 & $\mathrm{~S}$ & 5.32514400 & 1.40183600 & -0.13740500 \\
\hline $\mathrm{C}$ & -7.09003800 & 0.45338800 & -2.25806900 & $\mathrm{O}$ & 6.62150300 & 1.29254200 & 0.52346500 \\
\hline $\mathrm{C}$ & -5.86780300 & 0.11996200 & -2.85011300 & $\mathrm{O}$ & 5.20401900 & 1.86729500 & -1.51615300 \\
\hline $\mathrm{C}$ & -4.77122300 & -0.20727000 & -2.05217800 & $\mathrm{C}$ & 4.29381600 & 2.57173300 & 0.90275000 \\
\hline $\mathrm{C}$ & -3.32753900 & -2.50288000 & 0.28093800 & $\mathrm{~F}$ & 4.10098300 & 2.04764300 & 2.10975100 \\
\hline $\mathrm{C}$ & -4.42941500 & -3.29651700 & -0.07266100 & $\mathrm{~F}$ & 0600 & 2.77826300 & 0.30663300 \\
\hline $\mathrm{C}$ & -4.31635600 & -4.68940800 & -0.08896300 & $\mathrm{~F}$ & 4.95621800 & 3.72099100 & 1.00538600 \\
\hline $\mathrm{C}$ & -3.10801100 & -5.30121000 & 0.25152000 & $\mathrm{~s}$ & 4.66892900 & -1.20508000 & 1.10617300 \\
\hline 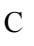 & -2.00669900 & -4.51681100 & 0.60712800 & $\mathrm{O}$ & 5.29691400 & -0.67908800 & 2.31355800 \\
\hline$c$ & -2.11286600 & -3.12559500 & 0.61582000 & $\mathrm{O}$ & 3.41991200 & -1.96146800 & 1.16137800 \\
\hline 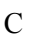 & -3.78427000 & -0.24600500 & 2.07651900 & $\mathrm{C}$ & 5.92116200 & -2.34156700 & 0.29938200 \\
\hline $\mathrm{C}$ & -3.41705200 & 1.02018900 & 2.56123100 & $\Gamma$ & 6.99590500 & -1.64559400 & -0.05846300 \\
\hline $\mathrm{C}$ & -3.69703500 & 1.37760300 & 3.88142700 & $\Gamma$ & 5.37072700 & -2.90918200 & 51100 \\
\hline $\mathrm{C}$ & -4.33709300 & 0.47291000 & 4.73230900 & $\mathrm{~F}$ & 6.25771500 & -3.27697500 & 1.18343800 \\
\hline 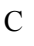 & -4.70039300 & -0.79028900 & 4.25847700 & & & & \\
\hline $\mathrm{C}$ & -4.42659500 & -1.15070700 & 400 & t 1 & & & \\
\hline $\mathrm{H}$ & -6.22817500 & 0.11690300 & 1.01592600 & $\mathrm{Au}$ & 0.10514700 & -0.10161400 & -0.12119000 \\
\hline $\mathrm{H}$ & -8.15885000 & 0.71303500 & -0.40177900 & $\mathrm{P}$ & -2.19283600 & -0.09390100 & -0.12220700 \\
\hline $\mathrm{H}$ & -7.94233400 & 0.71538200 & -2.87939700 & $\mathrm{C}$ & -2.86563500 & -1.33588000 & -1.28864400 \\
\hline$\Pi$ & -5.76564200 & 0.12204200 & -3.93200700 & $\mathrm{C}$ & -4.05801000 & -1.11738700 & -1.99542900 \\
\hline $\mathrm{H}$ & -3.81984500 & -0.45415400 & -2.51798000 & $\mathrm{C}$ & -4.54718700 & -2.09944500 & -2.85996500 \\
\hline $\mathrm{H}$ & -5.37571800 & -2.83370800 & -0.33535800 & $\mathrm{C}$ & -3.85446300 & -3.30125400 & -3.02280600 \\
\hline $\mathrm{H}$ & -5.17544500 & -5.29428200 & -0.36709400 & $\mathrm{C}$ & -2.66614000 & -3.52281700 & -2.32097900 \\
\hline $\mathrm{H}$ & -3.02283000 & -6.38455800 & 0.23655400 & $\mathrm{C}$ & -2.16904700 & -2.54376300 & -1.46051700 \\
\hline $\mathrm{H}$ & -1.06288200 & -4.9870 & 0 & $c$ & -2.87785 & 00 & 1.5309 \\
\hline $\mathrm{H}$ & -1.24872300 & -2.52007900 & 0.87988900 & $\mathrm{C}$ & -4.04488100 & -1.24785300 & 1.68595700 \\
\hline $\mathrm{H}$ & -2.91180400 & 1.72488700 & 1.90715200 & $\mathrm{C}$ & -4.54549300 & -1.50579000 & 2.96441600 \\
\hline $\mathrm{H}$ & -3.40774100 & 2.35990500 & 4.24548100 & $\mathrm{C}$ & -3.88838100 & -1.00582400 & 4.09072700 \\
\hline $\mathrm{H}$ & -4.54856600 & 0.74926600 & 5.76193300 & $\mathrm{C}$ & -2.72552200 & -0.24477200 & 3.94010300 \\
\hline $\mathrm{H}$ & -5.19565400 & -1.49906700 & 4.91686700 & $\mathrm{C}$ & -2.21691200 & 0.01297900 & 2.66690700 \\
\hline $\mathrm{H}$ & -4.71364300 & -2.13574800 & 2.58204200 & $\mathrm{C}$ & -2.86814200 & 1.53424300 & -0.61553300 \\
\hline $\mathrm{C}$ & -1.77895000 & 2.56439900 & -0.54747300 & $\mathrm{C}$ & -2.14901100 & 2.31029300 & -1.53954500 \\
\hline $\mathrm{C}$ & -0.97170500 & 3.42410600 & 0.20217400 & $\mathrm{C}$ & -2.64940500 & 3.54375800 & -1.95737300 \\
\hline $\mathrm{C}$ & -2.84295900 & 3.07570000 & -1.29450500 & $\mathrm{C}$ & -3.86413700 & 4.01460900 & -1.45117000 \\
\hline $\mathrm{C}$ & -1.24086400 & 4.80025100 & 0.21597700 & $\mathrm{C}$ & -4.57952700 & 3.24916500 & -0.52729700 \\
\hline $\mathrm{H}$ & -0.13112600 & 3.04157000 & 0.77770400 & $\mathrm{C}$ & -4.08616100 & 2.01183700 & -0.10760500 \\
\hline $\mathrm{C}$ & -3.10417100 & 4.45358400 & -1.28435900 & $\mathrm{H}$ & -4.60528000 & -0.18721500 & -1.87770600 \\
\hline $\mathrm{H}$ & -3.47723600 & 2.41912200 & -1.88565500 & $\mathrm{H}$ & -5.46893700 & -1.92169200 & -3.40727700 \\
\hline $\mathrm{C}$ & -2.30631300 & 5.31600900 & -0.52718200 & $\mathrm{H}$ & -4.23618300 & -4.06157400 & -3.69894300 \\
\hline
\end{tabular}




\begin{tabular}{|c|c|c|c|c|c|c|c|}
\hline $\mathrm{H}$ & -2.12067500 & -4.45385000 & -2.44874500 & $\mathrm{C}$ & 3.51434700 & -0.39464600 & 0.54085300 \\
\hline $\mathrm{H}$ & -1.23824300 & -2.71490500 & -0.92562100 & $\mathrm{C}$ & 4.82172800 & -0.05304500 & 0.18718200 \\
\hline $\mathrm{H}$ & -4.56347900 & -1.64299800 & 0.81800200 & $\mathrm{C}$ & 5.10543000 & 0.42374700 & -1.09415600 \\
\hline $\mathrm{H}$ & -5.44854100 & -2.09946400 & 3.07720700 & $\mathrm{C}$ & 4.07682600 & 0.56010700 & -2.03233500 \\
\hline $\mathrm{H}$ & -4.27887800 & -1.21103600 & 5.08388200 & $\mathrm{C}$ & 2.76711300 & 0.22604700 & -1.68752200 \\
\hline $\mathrm{H}$ & -2.20921300 & 0.14359400 & 4.81372100 & $\mathrm{C}$ & 0.65546100 & -1.08418700 & 1.78629900 \\
\hline $\mathrm{H}$ & -1.30918100 & 0.60023100 & 2.55556100 & $\mathrm{C}$ & 0.67658500 & -2.42733300 & 2.19632400 \\
\hline $\mathrm{H}$ & -1.19783000 & 1.95253900 & -1.92556000 & $\mathrm{C}$ & 0.63239800 & -2.73884900 & 3.55608400 \\
\hline $\mathrm{H}$ & -2.08678800 & 4.13900300 & -2.67133400 & $\mathrm{C}$ & 0.56711000 & -1.72010600 & 4.51003500 \\
\hline $\mathrm{H}$ & -4.24937200 & 4.97868900 & -1.77228200 & $\mathrm{C}$ & 0.54387100 & -0.38215300 & 4.10580000 \\
\hline $\mathrm{H}$ & -5.52212600 & 3.61425100 & -0.12868400 & $\mathrm{C}$ & 0.58247400 & -0.06202300 & 2.74846100 \\
\hline $\mathrm{H}$ & -4.64877800 & 1.42666100 & 0.61337600 & $\mathrm{C}$ & 0.18144000 & -2.04559800 & -0.96297500 \\
\hline $\mathrm{N}$ & 2.28108200 & -0.17191600 & -0.16119200 & $\mathrm{C}$ & 1.02812500 & -2.70306800 & -1.87101900 \\
\hline S & 3.08202500 & 0.62148800 & -1.37596900 & $\mathrm{C}$ & 0.54842500 & -3.78182100 & -2.61626500 \\
\hline $\mathrm{O}$ & 4.43476900 & 0.11685200 & -1.61695100 & $\mathrm{C}$ & -0.77096500 & -4.21259700 & -2.46408100 \\
\hline $\mathrm{O}$ & 2.14639700 & 0.77325400 & -2.49323900 & $\mathrm{C}$ & -1.61667400 & -3.56249200 & -1.55974600 \\
\hline $\mathrm{C}$ & 3.27653500 & 2.34046800 & -0.65840900 & $\mathrm{C}$ & -1.14932100 & -2.48042600 & -0.81581300 \\
\hline $\mathrm{F}$ & 3.86283000 & 2.26756100 & 0.53694300 & $\mathrm{H}$ & -5.26490900 & 0.18615900 & -2.07354800 \\
\hline $\mathrm{F}$ & 2.07661700 & 2.90653000 & -0.53423700 & $\mathrm{H}$ & -6.19654100 & -0.41204100 & 0.15619700 \\
\hline $\mathrm{F}$ & 4.03025000 & 3.06389200 & -1.48478300 & $\mathrm{H}$ & -4.75994800 & -0.22945600 & 2.18207400 \\
\hline S & 3.06811000 & -1.03414300 & 1.01538200 & $\mathrm{H}$ & -2.42001600 & 0.52399100 & 1.98489200 \\
\hline $\mathrm{O}$ & 4.47194000 & -0.65485000 & 1.18231300 & $\mathrm{H}$ & 3.30800700 & -0.76522700 & 1.53948000 \\
\hline $\mathrm{O}$ & 2.18081200 & -1.09377500 & 2.18006300 & $\mathrm{H}$ & 5.61839600 & -0.16117500 & 0.91801500 \\
\hline $\mathrm{C}$ & 3.07257300 & -2.76544200 & 0.30382200 & $\mathrm{H}$ & 6.12377900 & 0.69082500 & -1.36238800 \\
\hline $\mathrm{F}$ & 3.64291800 & -2.75584600 & -0.89994600 & $\mathrm{H}$ & 4.29190600 & 0.92982900 & -3.03092300 \\
\hline $\mathrm{F}$ & 1.81896500 & -3.20937400 & 0.20158700 & $\mathrm{H}$ & 1.97201500 & 0.33533400 & -2.42179200 \\
\hline $\mathrm{F}$ & 3.76263800 & -3.55775500 & 1.12255400 & $\mathrm{H}$ & 0.73092500 & -3.22577700 & 1.46323300 \\
\hline C & 0.41682800 & 3.09165600 & 3.42494500 & $\mathrm{H}$ & 0.65027900 & -3.77940000 & 3.86814300 \\
\hline $\mathrm{H}$ & -0.59437100 & 3.50256500 & 3.35705200 & $\mathrm{H}$ & 0.53087100 & -1.96824700 & 5.56731600 \\
\hline $\mathrm{H}$ & 0.45251100 & 2.38153500 & 4.26221000 & $\mathrm{H}$ & 0.48933200 & 0.41175500 & 4.84545200 \\
\hline $\mathrm{H}$ & 1.11712700 & 3.91389500 & 3.62842400 & $\mathrm{H}$ & 0.55474500 & 0.97850800 & 2.43488600 \\
\hline $\mathrm{O}$ & 0.69662100 & 2.45818900 & 2.17479500 & $\mathrm{H}$ & 2.05812400 & -2.38721100 & -1.99506800 \\
\hline \multirow[t]{2}{*}{$\mathrm{H}$} & 1.57199400 & 2.04364300 & 2.24110300 & $\mathrm{H}$ & 1.21189100 & -4.28638600 & -3.31321400 \\
\hline & & & & $\mathrm{H}$ & -1.14020900 & -5.05200100 & -3.04691400 \\
\hline \multicolumn{2}{|c|}{ Int $10^{\prime}$} & & & $\mathrm{H}$ & -2.64370400 & -3.89446300 & -1.43605100 \\
\hline \multicolumn{2}{|c|}{$\mathrm{Au}-0.61611700$} & 1.53983800 & -0.34072600 & $\mathrm{H}$ & -1.81469900 & -1.98138300 & -0.11813100 \\
\hline $\mathrm{P}$ & 0.74216200 & -0.62261400 & 0.02727800 & $\mathrm{H}$ & -2.93011400 & 0.94960300 & -2.28436800 \\
\hline $\mathrm{C}$ & -2.50827400 & 0.81691900 & -0.16120200 & $\mathrm{C}$ & 1.74391400 & 3.52554800 & 0.54050800 \\
\hline $\mathrm{C}$ & -3.31997800 & 0.69949300 & -1.30111700 & $\mathrm{H}$ & 0.92851500 & 4.07963100 & 1.00748800 \\
\hline $\mathrm{C}$ & -4.64527400 & 0.26587100 & -1.18330700 & $\mathrm{H}$ & 2.13539000 & 2.77945200 & 1.23885000 \\
\hline $\mathrm{C}$ & -5.16883600 & -0.06880600 & 0.06817000 & $\mathrm{H}$ & 2.53992400 & 4.21731700 & 0.24832600 \\
\hline $\mathrm{C}$ & -4.36192200 & 0.03266600 & 1.20445500 & $\mathrm{O}$ & 1.19823900 & 2.89710000 & -0.64174100 \\
\hline $\mathrm{C}$ & -3.03567800 & 0.46530100 & 1.09134100 & $\mathrm{H}$ & 1.90096600 & 2.36668400 & -1.06138700 \\
\hline C & 2.47833800 & -0.26374300 & -0.39738300 & & & & \\
\hline
\end{tabular}




\begin{tabular}{|c|c|c|c|c|c|c|c|}
\hline \multicolumn{4}{|c|}{ Int10" } & \multirow{2}{*}{$\begin{array}{l}\mathrm{H} \\
\mathrm{H}\end{array}$} & \multirow{2}{*}{$\begin{array}{l}-5.65120300 \\
-3.93190400\end{array}$} & \multirow{2}{*}{$\begin{array}{l}-1.86263500 \\
-1.08592900\end{array}$} & \multirow{2}{*}{$\begin{array}{l}1.66889300 \\
0.08490200\end{array}$} \\
\hline $\mathrm{Au}$ & 0.02863100 & -0.88838000 & -0.06738500 & & & & \\
\hline $\mathrm{P}$ & -1.82260400 & 0.93547400 & 0.25915700 & $\mathrm{H}$ & -1.11713900 & -3.07297400 & 1.79610300 \\
\hline $\mathrm{C}$ & -1.29475500 & -2.43599800 & -0.26887100 & $N$ & 2.06351400 & 0.20401700 & 0.08015400 \\
\hline $\mathrm{C}$ & -1.55278700 & -3.27994600 & 0.82184900 & $\mathrm{~S}$ & 2.92557900 & -0.00952000 & 1.47035600 \\
\hline $\mathrm{C}$ & -2.37041000 & -4.40550700 & 0.66475800 & $\mathrm{O}$ & 4.09510400 & 0.86426700 & 1.59364000 \\
\hline C & -2.95012300 & -4.68793400 & -0.57469100 & $\mathrm{O}$ & 1.96184900 & -0.09750700 & 2.57245300 \\
\hline $\mathrm{C}$ & -2.70911000 & -3.84055400 & -1.65956500 & $\mathrm{C}$ & 3.60472000 & -1.75113800 & 1.30510600 \\
\hline $\mathrm{C}$ & -1.89088600 & -2.71432200 & -1.50755000 & $\mathrm{~F}$ & 4.26224800 & -1.88558500 & 0.14852400 \\
\hline $\mathrm{C}$ & -1.16166400 & 2.53761100 & 0.83590000 & $\mathrm{~F}$ & 2.60382400 & -2.63264700 & 1.34840800 \\
\hline $\mathrm{C}$ & -1.57996600 & 3.75103500 & 0.26498900 & $\mathrm{~F}$ & 4.43949100 & -1.97673200 & 2.31761800 \\
\hline $\mathrm{C}$ & -1.03940000 & 4.95585500 & 0.71677500 & $\mathrm{~S}$ & 2.76104700 & 0.80849200 & -1.29149500 \\
\hline $\mathrm{C}$ & -0.07945700 & 4.96058300 & 1.73200300 & $\mathrm{O}$ & 4.22144300 & 0.68690000 & -1.32677400 \\
\hline $\mathrm{C}$ & 0.34130000 & 3.75518200 & 2.30050200 & $\mathrm{O}$ & 1.96598900 & 0.33571000 & -2.42993400 \\
\hline $\mathrm{C}$ & -0.19092600 & 2.54575900 & 1.85208600 & $c$ & 2.40885400 & 2.64607500 & -1.17641600 \\
\hline $\mathrm{C}$ & -2.60694700 & 1.21362600 & -1.36545400 & $\mathrm{~F}$ & 2.77541000 & 3.11039900 & 0.01735100 \\
\hline $\mathrm{C}$ & -3.95755800 & 1.58234800 & -1.48747500 & $\mathrm{~F}$ & 1.10752500 & 2.86384600 & -1.36333100 \\
\hline $\mathrm{C}$ & -4.50500700 & 1.81551200 & -2.75040400 & $\Gamma$ & 3.10428000 & 3.26546100 & -2.13065100 \\
\hline $\mathrm{C}$ & -3.71535300 & 1.68307000 & -3.89549700 & $\mathrm{C}$ & 1.76102100 & -4.40855400 & -1.67001100 \\
\hline $\mathrm{C}$ & -2.37122300 & 1.31917600 & -3.77893600 & $\mathrm{H}$ & 0.78993400 & -4.65027600 & -2.10950400 \\
\hline $\mathrm{C}$ & -1.81683600 & 1.08191800 & -2.52054600 & $\mathrm{H}$ & 2.52196700 & -5.06241300 & -2.11890900 \\
\hline $\mathrm{C}$ & -3.12382900 & 0.41376700 & 1.42819900 & $\mathrm{H}$ & 1.71821800 & -4.60561100 & -0.59060000 \\
\hline $\mathrm{C}$ & -3.22937800 & 0.98948900 & 2.70553000 & $\mathrm{O}$ & 2.02065900 & -3.03144700 & -1.95122700 \\
\hline $\mathrm{C}$ & -4.20219900 & 0.53830500 & 3.59923500 & $\mathrm{H}$ & 2.86334400 & -2.80185800 & -1.52801800 \\
\hline $\mathrm{C}$ & -5.07556400 & -0.48725300 & 3.23121500 & & & & \\
\hline $\mathrm{C}$ & -4.97379800 & -1.06538200 & 1.96219200 & $\mathrm{I}_{1}$ & & & \\
\hline $\mathrm{C}$ & -4.00059700 & -0.62633000 & 1.06558200 & & -0.59495600 & 1.28853000 & 0.33767700 \\
\hline $\mathrm{H}$ & -2.55540400 & -5.05713500 & 1.51581800 & $\mathrm{P}$ & 0.91564900 & -0.47128200 & -0.05820800 \\
\hline $\mathrm{H}$ & -3.58949400 & -5.55912000 & -0.69325800 & $\mathrm{C}$ & 0.83238000 & -0.95332500 & -1.79864800 \\
\hline $\mathrm{H}$ & -3.15916400 & -4.05076800 & -2.62725200 & $\mathrm{C}$ & 1.04634300 & -2.28286500 & -2.19547300 \\
\hline $\mathrm{H}$ & -1.72215100 & -2.06282800 & -2.36079800 & $\mathrm{C}$ & 1.01597700 & -2.60669600 & -3.55279000 \\
\hline $\mathrm{H}$ & -2.32262800 & 3.76058300 & -0.52612600 & $\mathrm{C}$ & 0.77473200 & -1.61671600 & -4.50766600 \\
\hline $\mathrm{H}$ & -1.36932700 & 5.89087300 & 0.27216500 & $\mathrm{C}$ & 0.56694600 & -0.29110200 & -4.11229100 \\
\hline $\mathrm{H}$ & 0.34223300 & 5.90080300 & 2.07695600 & $\mathrm{C}$ & 0.59308500 & 0.04481500 & -2.76059000 \\
\hline $\mathrm{H}$ & 1.09143700 & 3.75321200 & 3.08664500 & $\mathrm{C}$ & 2.46057400 & 0.42365700 & 0.26938100 \\
\hline $\mathrm{H}$ & 0.15528500 & 1.61259100 & 2.28570000 & $\mathrm{C}$ & 3.49911800 & 0.42040300 & -0.67778400 \\
\hline $\mathrm{H}$ & -4.58082000 & 1.69009700 & -0.60548900 & $c$ & 4.67292900 & 1.12383000 & -0.41186500 \\
\hline $\mathrm{H}$ & -5.55002300 & 2.10020000 & -2.83679300 & $\mathrm{C}$ & 4.81245500 & 1.83791000 & 0.78171300 \\
\hline $\mathrm{H}$ & -4.14738400 & 1.86044900 & -4.87669800 & C & 3.77841700 & 1.84817300 & 1.72216400 \\
\hline $\mathrm{H}$ & -1.75438400 & 1.21196100 & -4.66684500 & $\mathrm{C}$ & 2.60027800 & 1.14662200 & 1.47142400 \\
\hline $\mathrm{H}$ & -0.77351800 & 0.79070400 & -2.43772500 & $\mathrm{C}$ & 0.74371200 & -1.90761400 & 1.02940100 \\
\hline $\mathrm{H}$ & -2.56443500 & 1.79302400 & 3.00392500 & $\mathrm{C}$ & -0.35211700 & -2.77553000 & 0.86336200 \\
\hline $\mathrm{H}$ & -4.27829700 & 0.99593100 & 4.58179300 & $\mathrm{C}$ & -0.48169800 & -3.88779200 & 1.69157000 \\
\hline $\mathrm{H}$ & -5.83267300 & -0.83506700 & 3.92877500 & $\mathrm{C}$ & 0.46723300 & -4.13771800 & 2.68806100 \\
\hline
\end{tabular}




\begin{tabular}{|c|c|c|c|c|c|c|c|}
\hline $\mathrm{C}$ & 1.55504800 & -3.27809900 & 2.85206100 & $\mathrm{C}$ & -0.55839100 & 2.00148900 & 1.38598500 \\
\hline $\mathrm{C}$ & 1.70001200 & -2.16239200 & 2.02685400 & $\mathrm{C}$ & -0.58804300 & 3.38061600 & 1.12140300 \\
\hline $\mathrm{H}$ & 1.23718100 & -3.05789800 & -1.46099000 & $\mathrm{C}$ & 0.15335900 & 4.25253000 & 1.91976600 \\
\hline $\mathrm{H}$ & 1.18029300 & -3.63522900 & -3.86044800 & $\mathrm{C}$ & 0.92692100 & 3.75970600 & 2.97260700 \\
\hline $\mathrm{H}$ & 0.74801100 & -1.87683800 & -5.56212300 & $\mathrm{C}$ & 0.96412200 & 2.38669900 & 3.23107600 \\
\hline $\mathrm{H}$ & 0.38022700 & 0.47902900 & -4.85485500 & $\mathrm{C}$ & 0.22768000 & 1.50582600 & 2.44004700 \\
\hline $\mathrm{H}$ & 0.42813300 & 1.07333500 & -2.45008500 & $\mathrm{C}$ & -2.22951900 & 1.71576400 & -1.05919600 \\
\hline $\mathrm{H}$ & 3.39868100 & -0.12930000 & -1.60738700 & $\mathrm{C}$ & -3.34325900 & 2.56693800 & -0.93996900 \\
\hline $\mathrm{H}$ & 5.47855000 & 1.11372700 & -1.14027600 & $\mathrm{C}$ & -3.80263100 & 3.26569900 & -2.05639300 \\
\hline $\mathrm{H}$ & 5.72848100 & 2.38756400 & 0.97909300 & $\mathrm{C}$ & -3.16287200 & 3.12019400 & -3.28983700 \\
\hline $\mathrm{H}$ & 3.88686600 & 2.40270600 & 2.64948900 & $\mathrm{C}$ & -2.05579400 & 2.27690600 & -3.40975800 \\
\hline $\mathrm{H}$ & 1.79909700 & 1.15306600 & 2.20636100 & $\mathrm{C}$ & -1.58634100 & 1.57516600 & -2.29936700 \\
\hline $\mathrm{H}$ & -1.08947600 & -2.59415700 & 0.08858500 & $\mathrm{C}$ & -3.01533300 & 0.36922000 & 1.44552000 \\
\hline $\mathrm{H}$ & -1.32528200 & -4.55851600 & 1.55813300 & $\mathrm{C}$ & -2.93778600 & 0.42163500 & 2.84708100 \\
\hline $\mathrm{H}$ & 0.35873600 & -5.00508800 & 3.33305800 & $\mathrm{C}$ & -4.03690100 & 0.04448100 & 3.61969200 \\
\hline $\mathrm{H}$ & 2.29728500 & -3.47417400 & 3.62015500 & $\mathrm{C}$ & -5.21647300 & -0.37970400 & 3.00562800 \\
\hline $\mathrm{H}$ & 2.55468900 & -1.50830900 & 2.15869000 & $\mathrm{C}$ & -5.29811700 & -0.43062500 & 1.61106900 \\
\hline $\mathrm{C}$ & -2.21996600 & 0.05458000 & 0.16066400 & $\mathrm{C}$ & -4.20364900 & -0.06676000 & 0.82992200 \\
\hline $\mathrm{C}$ & -2.84692600 & -0.30789100 & 1.34584400 & $\mathrm{H}$ & -3.81283200 & -4.37139000 & 0.85038000 \\
\hline 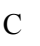 & -2.68439100 & -0.27633200 & -1.10275600 & $\mathrm{H}$ & -4.91698900 & -4.30929200 & -1.37977700 \\
\hline$C$ & -4.04029300 & -1.03548400 & 1.24278500 & $\mathrm{H}$ & -4.10040200 & -2.73753900 & -3.12801100 \\
\hline $\mathrm{H}$ & -2.44298300 & -0.04104200 & 2.31653600 & $\mathrm{H}$ & -2.19024200 & -1.22913300 & -2.65441600 \\
\hline $\mathrm{C}$ & -3.88040200 & -1.00575900 & -1.17469600 & $\mathrm{H}$ & -1.18211600 & 3.77814400 & 0.30632900 \\
\hline$\Pi$ & -2.15451200 & 0.00666400 & -2.00528900 & $\mathrm{H}$ & 0.12568700 & 5.31848900 & 1.71280200 \\
\hline $\mathrm{C}$ & -4.55138300 & -1.38248200 & -0.00976200 & $\mathrm{H}$ & 1.50482600 & 4.44333600 & 3.58821000 \\
\hline $\mathrm{H}$ & -4.55873100 & -1.32767100 & 2.15183900 & $\mathrm{H}$ & 1.57056300 & 1.99777900 & 4.04362400 \\
\hline$\Pi$ & -4.27369900 & -1.27626000 & -2.15069800 & $\mathrm{H}$ & 0.26772300 & 0.44082200 & 2.64205700 \\
\hline $\mathrm{H}$ & -5.47413200 & -1.95155000 & -0.07795400 & $\mathrm{H}$ & -3.84822700 & 2.68911500 & 0.01252200 \\
\hline $\mathrm{C}$ & -2.03355400 & 4.05021500 & -0.34964700 & $\mathrm{H}$ & -4.66315400 & 3.92116900 & -1.95919700 \\
\hline $\mathrm{H}$ & -1.06093300 & 4.35470700 & -0.73677800 & $\mathrm{H}$ & -3.52853300 & 3.66244200 & -4.15737100 \\
\hline $\mathrm{H}$ & -2.61575000 & 3.55276500 & -1.13021900 & $\mathrm{H}$ & -1.55731600 & 2.15961400 & -4.36759000 \\
\hline П & -2.57255100 & 4.91956500 & 0.03540800 & $\mathrm{H}$ & -0.72991500 & 0.91711700 & -2.40031700 \\
\hline 0 & -1.77357100 & 3.14268200 & 0.75476600 & $\mathrm{H}$ & -2.03793400 & 0.76424600 & 3.34360900 \\
\hline \multirow[t]{2}{*}{$\mathrm{H}$} & -2.62390800 & 2.87232500 & 1.15032900 & $\mathrm{H}$ & -3.96860400 & 0.09142600 & 4.70266500 \\
\hline & & & & $\mathrm{H}$ & -6.07158900 & -0.66790600 & 3.61076600 \\
\hline \multicolumn{2}{|c|}{ Int11" } & & & $\mathrm{H}$ & -6.21395500 & -0.75783400 & 1.12751500 \\
\hline \multicolumn{2}{|c|}{$\mathrm{Au}-0.20085000$} & -0.95727700 & -0.32230000 & $\mathrm{H}$ & -4.27980700 & -0.12303600 & -0.25027000 \\
\hline$P$ & -1.59420100 & 0.86144900 & 0.41351500 & $\mathrm{H}$ & -1.91532400 & -2.85434400 & 1.34430800 \\
\hline $\mathrm{C}$ & -1.94775700 & -1.97661600 & -0.63672100 & $\mathrm{~N}$ & 1.98833500 & -0.17008900 & -0.13895200 \\
\hline $\mathrm{C}$ & -2.38600700 & -2.83598600 & 0.36641200 & $\mathrm{~S}$ & 2.86918400 & -0.57973300 & 1.21144800 \\
\hline $\mathrm{C}$ & -3.46138500 & -3.68678700 & 0.08301800 & $\mathrm{O}$ & 3.64117900 & 0.53230900 & 1.77057600 \\
\hline $\mathrm{C}$ & -4.07813600 & -3.65128600 & -1.17015700 & 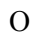 & 1.93949800 & -1.33437400 & 2.06129300 \\
\hline $\mathrm{C}$ & -3.62232200 & -2.76983200 & -2.15261800 & $\mathrm{C}$ & 4.17356900 & -1.86316100 & 0.66730800 \\
\hline $\mathrm{C}$ & -2.54292700 & -1.91466600 & -1.89133600 & $\mathrm{~F}$ & 3.88762200 & -2.36770800 & -0.53483200 \\
\hline
\end{tabular}




\begin{tabular}{|c|c|c|c|c|c|c|c|}
\hline $\mathrm{F}$ & 4.15683300 & -2.84008600 & 1.57198100 & $\mathrm{C}$ & -2.39049700 & 3.52305000 & -1.77436600 \\
\hline $\mathrm{F}$ & 5.37218200 & -1.29829000 & 0.64180900 & $\mathrm{C}$ & -2.06198600 & 2.18605500 & -1.55015200 \\
\hline S & 2.72911000 & 0.61668500 & -1.38921000 & $\mathrm{C}$ & -2.55593300 & -1.05946300 & -1.20797700 \\
\hline $\mathrm{O}$ & 4.18741100 & 0.46367200 & -1.33530400 & $\mathrm{C}$ & -1.92992400 & -2.10562100 & -1.90382100 \\
\hline $\mathrm{O}$ & 1.98988900 & 0.28492900 & -2.61181600 & $\mathrm{C}$ & -2.64726000 & -2.85952800 & -2.83523400 \\
\hline $\mathrm{C}$ & 2.42120000 & 2.44911100 & -1.11670500 & $\mathrm{C}$ & -3.99149100 & -2.57297700 & -3.08123600 \\
\hline $\mathrm{F}$ & 2.70098800 & 2.78676300 & 0.13765300 & $\mathrm{C}$ & -4.61918000 & -1.52689200 & -2.39844300 \\
\hline $\mathrm{F}$ & 1.14919500 & 2.73483600 & -1.39057300 & $\mathrm{C}$ & -3.90708100 & -0.76864500 & -1.46872200 \\
\hline $\mathrm{F}$ & 3.21531500 & 3.11823100 & -1.95197400 & $\mathrm{H}$ & -4.01877200 & -1.45782800 & 1.13964000 \\
\hline $\mathrm{C}$ & 0.99553700 & -3.97584400 & -0.39265100 & $\mathrm{H}$ & -4.64415600 & -2.03623900 & 3.45275600 \\
\hline $\mathrm{H}$ & 0.02038200 & -4.33776700 & -0.06740500 & $\mathrm{H}$ & -3.12492500 & -1.47925000 & 5.34132600 \\
\hline $\mathrm{H}$ & 1.48899700 & -4.73865500 & -1.00084900 & $\mathrm{H}$ & -0.95969700 & -0.33803600 & 4.89401400 \\
\hline $\mathrm{H}$ & 1.61019000 & -3.72311800 & 0.47432000 & $\mathrm{H}$ & -0.31615300 & 0.23808900 & 2.57837500 \\
\hline $\mathrm{O}$ & 0.75000700 & -2.81348600 & -1.22508900 & $\mathrm{H}$ & -2.55208300 & 2.08346500 & 1.82694700 \\
\hline $\mathrm{H}$ & 1.58213500 & -2.54355000 & -1.65288100 & $\mathrm{H}$ & -3.13268000 & 4.44352800 & 1.41785500 \\
\hline & & & & $\mathrm{H}$ & -3.03057100 & 5.38182100 & -0.88461500 \\
\hline Int 1 & & & & $\mathrm{H}$ & -2.35119500 & 3.92310100 & -2.78388400 \\
\hline $\mathrm{C}$ & 1.43148600 & 2.36626100 & -0.04158000 & $\mathrm{H}$ & -1.77547300 & 1.55916000 & -2.39036900 \\
\hline $\mathrm{O}$ & 1.45063500 & 3.73388900 & -0.81797900 & $\mathrm{H}$ & -0.88246000 & -2.32676900 & -1.72517000 \\
\hline $\mathrm{C}$ & 2.54767500 & 4.61709100 & -0.28643900 & $\mathrm{H}$ & -2.15258500 & -3.66535500 & -3.37049700 \\
\hline $\mathrm{C}$ & 2.81658900 & 4.01486400 & 1.07784400 & $\mathrm{H}$ & -4.54837700 & -3.15842300 & -3.80787200 \\
\hline $\mathrm{C}$ & 2.65649700 & 2.50354700 & 0.84906100 & $\mathrm{H}$ & -5.66316600 & -1.29661000 & -2.59250100 \\
\hline $\mathrm{H}$ & 0.49903800 & 2.46562200 & 0.51087700 & $\mathrm{H}$ & -4.40227300 & 0.04911200 & -0.95374200 \\
\hline $\mathrm{H}$ & 3.38626700 & 4.52719100 & -0.97736400 & $\mathrm{C}$ & 2.92047700 & -1.01563100 & -0.25713800 \\
\hline $\mathrm{H}$ & 2.13594700 & 5.62518400 & -0.28886200 & $\mathrm{C}$ & 3.51901900 & -1.39601300 & -1.46220300 \\
\hline $\mathrm{H}$ & 3.81932700 & 4.27874400 & 1.42372700 & $\mathrm{C}$ & 3.65823700 & -1.04466200 & 0.93106800 \\
\hline $\mathrm{H}$ & 2.08727100 & 4.38124000 & 1.80731400 & $\mathrm{C}$ & 4.84961500 & -1.83221800 & -1.47191300 \\
\hline $\mathrm{H}$ & 3.53730800 & 2.09179700 & 0.34666500 & $\mathrm{H}$ & 2.96083600 & -1.36390000 & -2.39447800 \\
\hline $\mathrm{H}$ & 2.50335700 & 1.96103300 & 1.78549700 & $\mathrm{C}$ & 4.99005800 & -1.47658200 & 0.91492900 \\
\hline $\mathrm{C}$ & 1.39339800 & 1.26801500 & -1.07137400 & $\mathrm{H}$ & 3.20657900 & -0.74276100 & 1.87238500 \\
\hline $\mathrm{H}$ & 0.57982100 & 1.41210000 & -1.78606000 & $\mathrm{C}$ & 5.58595700 & -1.87201600 & -0.28539600 \\
\hline $\mathrm{H}$ & 2.34564900 & 1.16708100 & -1.59133600 & $\mathrm{H}$ & 5.30805000 & -2.13745300 & -2.40955200 \\
\hline $\mathrm{Au}$ & 0.91208800 & -0.55212700 & -0.17544300 & $\mathrm{H}$ & 5.55791300 & -1.50105300 & 1.84199500 \\
\hline $\mathrm{P}$ & -1.58810600 & -0.08950800 & 0.00614400 & $\mathrm{H}$ & 6.62022400 & -2.20591600 & -0.29641600 \\
\hline $\mathrm{C}$ & -2.12810200 & -0.55871600 & 1.69428600 & $\mathrm{H}$ & 1.52646100 & 3.60959900 & -1.78951100 \\
\hline $\mathrm{C}$ & -3.34627100 & -1.20512300 & 1.95267400 & $\mathrm{C}$ & 1.23620200 & -3.74408300 & 0.63397300 \\
\hline $\mathrm{C}$ & -3.69963800 & -1.53362400 & 3.26310300 & $\mathrm{H}$ & 1.45544700 & -3.90551000 & -0.42149300 \\
\hline $\mathrm{C}$ & -2.84568700 & -1.22094400 & 4.32348600 & $\mathrm{H}$ & 2.16280300 & -3.58260700 & 1.19003600 \\
\hline $\mathrm{C}$ & -1.62925100 & -0.58059600 & 4.07339400 & $\mathrm{H}$ & 0.69767500 & -4.60668200 & 1.03716000 \\
\hline $\mathrm{C}$ & -1.26685600 & -0.25579500 & 2.76494300 & $\mathrm{O}$ & 0.36917000 & -2.58664500 & 0.71202300 \\
\hline $\mathrm{C}$ & -2.11423900 & 1.65121700 & -0.24976200 & $\mathrm{H}$ & 0.15784900 & -2.41767600 & 1.64854300 \\
\hline $\mathrm{C}$ & -2.50002600 & 2.47711400 & 0.81695200 & & & & \\
\hline $\mathrm{C}$ & -2.82894600 & 3.81540800 & 0.58481700 & \multicolumn{4}{|c|}{$\mathrm{TS} 2^{\prime}$} \\
\hline $\mathrm{C}$ & -2.77368200 & 4.34099300 & -0.70742500 & $\mathrm{C}$ & 1.93064000 & 2.60409200 & -0.11332400 \\
\hline
\end{tabular}




\begin{tabular}{|c|c|c|c|c|c|c|c|}
\hline $\mathrm{O}$ & 2.69800200 & 3.72041000 & -0.63109000 & $\mathrm{H}$ & -1.07973700 & -2.72677400 & -0.81409600 \\
\hline C & 3.58671900 & 4.19969800 & 0.39810600 & $\mathrm{H}$ & -2.23233100 & -4.33685600 & -2.29352300 \\
\hline $\mathrm{C}$ & 2.84773400 & 3.91641900 & 1.70040600 & $\mathrm{H}$ & -4.28866500 & -3.65799800 & -3.51949800 \\
\hline C & 2.21756100 & 2.54539100 & 1.40241500 & $\mathrm{H}$ & -5.18715300 & -1.35495100 & -3.25076600 \\
\hline $\mathrm{H}$ & 0.88030200 & 2.81847800 & -0.32552000 & $\mathrm{H}$ & -4.04513300 & 0.26160900 & -1.77443200 \\
\hline $\mathrm{H}$ & 4.54593500 & 3.66406100 & 0.35438400 & $\mathrm{C}$ & 2.77016600 & -0.79498200 & -0.56345000 \\
\hline $\mathrm{H}$ & 3.76887700 & 5.26005000 & 0.19905400 & $\mathrm{C}$ & 3.11815500 & -1.38112500 & -1.78670300 \\
\hline $\mathrm{H}$ & 3.51003200 & 3.90168100 & 2.57125800 & $\mathrm{C}$ & 3.47021200 & -1.10865800 & 0.60908400 \\
\hline $\mathrm{H}$ & 2.07319800 & 4.67437000 & 1.86467100 & $\mathrm{C}$ & 4.13681300 & -2.33834800 & -1.82118600 \\
\hline $\mathrm{H}$ & 2.93144900 & 1.74444200 & 1.62281800 & $\mathrm{H}$ & 2.60099300 & -1.10346100 & -2.70058800 \\
\hline $\mathrm{H}$ & 1.30795000 & 2.35682600 & 1.97904000 & $\mathrm{C}$ & 4.48571600 & -2.06783800 & 0.56109100 \\
\hline $\mathrm{C}$ & 2.42313300 & 1.41276900 & -0.92903400 & $\mathrm{H}$ & 3.22520000 & -0.62322200 & 1.54865700 \\
\hline $\mathrm{H}$ & 2.14669700 & 1.43740100 & -1.98319400 & $\mathrm{C}$ & 4.81934400 & -2.68160500 & -0.65062700 \\
\hline $\mathrm{H}$ & 3.49469900 & 1.29424900 & -0.81027600 & $\mathrm{H}$ & 4.39826200 & -2.80936800 & -2.76525100 \\
\hline A & 0.80694200 & -0.13631000 & -0.35359200 & $\mathrm{H}$ & 5.02119300 & -2.32737900 & 1.47071800 \\
\hline $\mathrm{P}$ & -1.59319700 & 0.02810000 & -0.06978600 & $\mathrm{H}$ & 5.61779900 & -3.41802800 & -0.68400300 \\
\hline $\mathrm{C}$ & -2.11030500 & -0.42088000 & 1.62944000 & $\mathrm{C}$ & 1.34723100 & -3.87298300 & 2.17264900 \\
\hline$c$ & -3.41143500 & -0.86884800 & 1.91094400 & $\mathrm{H}$ & 2.08029800 & -4.32875800 & 1.50204600 \\
\hline $\mathrm{C}$ & -3.76876700 & -1.20130400 & 3.21875000 & $\mathrm{H}$ & 1.87752600 & -3.24550900 & 2.90143900 \\
\hline 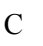 & -2.83345000 & -1.09705400 & 4.25209700 & $\mathrm{H}$ & 0.82067700 & -4.67254100 & 2.71208100 \\
\hline $\mathrm{C}$ & -1.53598300 & -0.65763300 & 3.97809900 & $\mathrm{O}$ & 0.45143600 & -3.10912900 & 1.36182800 \\
\hline $\mathrm{C}$ & -1.17295000 & -0.32217500 & 2.67173300 & $\mathrm{H}$ & -0.18818200 & -2.67381600 & 1.94985400 \\
\hline $\mathrm{C}$ & -2.24420600 & 1.70528700 & -0.42461000 & & & & \\
\hline $\mathrm{C}$ & -3.20251400 & 2.32866100 & 0.38770700 & & & & \\
\hline $\mathrm{C}$ & -3.66670100 & 3.60690000 & 0.06589400 & & & & \\
\hline $\mathrm{C}$ & -3.18359900 & 4.26741500 & -1.06525000 & & & & \\
\hline $\mathrm{C}$ & -2.22893600 & 3.64979200 & -1.87908200 & & & & \\
\hline $\mathrm{C}$ & -1.75526200 & 2.37750100 & -1.55867900 & & & & \\
\hline $\mathrm{C}$ & -2.48216000 & -1.11933800 & -1.19282800 & & & & \\
\hline $\mathrm{C}$ & -1.97555400 & -2.42120700 & -1.34902600 & & & & \\
\hline $\mathrm{C}$ & -2.62886700 & -3.33154700 & -2.17985600 & & & & \\
\hline $\mathrm{C}$ & -3.78483500 & -2.94954000 & -2.86744800 & & & & \\
\hline $\mathrm{C}$ & -4.28931700 & -1.65624100 & -2.71795900 & & & & \\
\hline $\mathrm{C}$ & -3.64306500 & -0.74087600 & -1.88292300 & & & & \\
\hline $\mathrm{H}$ & -4.14436000 & -0.96049500 & 1.11466200 & & & & \\
\hline $\mathrm{H}$ & -4.77747700 & -1.54634200 & 3.42865400 & & & & \\
\hline $\mathrm{H}$ & -3.11412900 & -1.36258800 & 5.26772900 & & & & \\
\hline $\mathrm{H}$ & -0.80389900 & -0.58023500 & 4.77731400 & & & & \\
\hline $\mathrm{H}$ & -0.16092900 & 0.01231400 & 2.45874300 & & & & \\
\hline $\mathrm{H}$ & -3.58922800 & 1.82659800 & 1.26874600 & & & & \\
\hline $\mathrm{H}$ & -4.40729200 & 4.08335600 & 0.70250000 & & & & \\
\hline $\mathrm{H}$ & -3.54617100 & 5.26174600 & -1.31145500 & & & & \\
\hline $\mathrm{H}$ & -1.84771700 & 4.16026600 & -2.75919900 & & & & \\
\hline $\mathrm{H}$ & -1.00718000 & 1.90617100 & -2.19142400 & & & & \\
\hline
\end{tabular}

